\title{
Exponentially Small Splitting of Separatrices Associated to 3D Whiskered Tori with Cubic Frequencies
}

\author{
Amadeu Delshams ${ }^{1,2}$ (D), Marina Gonchenko ${ }^{1}$ (D), Pere Gutiérrez ${ }^{1}$ (D) \\ 1 Dep. de Matemàtiques, Univ. Politècnica de Catalunya, Av. Diagonal 647, 08028 Barcelona, Spain. \\ E-mail: amadeu.delshams@upc.edu,marina.gonchenko@upc.edu,pere.gutierrez@upc.edu \\ 2 Lab of Geometry and Dynamical Systems, Univ. Politècnica de Catalunya, Av. Dr. Marañón 44-50, 08028 \\ Barcelona, Spain
}

Received: 30 May 2019 / Accepted: 1 June 2020

(C) Springer-Verlag GmbH Germany, part of Springer Nature 2020

\begin{abstract}
We study the splitting of invariant manifolds of whiskered (hyperbolic) tori with three frequencies in a nearly-integrable Hamiltonian system, whose hyperbolic part is given by a pendulum. We consider a 3-dimensional torus with a fast frequency vector $\omega / \sqrt{\varepsilon}$, with $\omega=(1, \Omega, \widetilde{\Omega})$ where $\Omega$ is a cubic irrational number whose two conjugates are complex, and the components of $\omega$ generate the field $\mathbb{Q}(\Omega)$. A paradigmatic case is the cubic golden vector, given by the (real) number $\Omega$ satisfying $\Omega^{3}=1-\Omega$, and $\widetilde{\Omega}=\Omega^{2}$. For such 3-dimensional frequency vectors, the standard theory of continued fractions cannot be applied, so we develop a methodology for determining the behavior of the small divisors $\langle k, \omega\rangle, k \in \mathbb{Z}^{3}$. Applying the Poincaré-Melnikov method, this allows us to carry out a careful study of the dominant harmonic (which depends on $\varepsilon$ ) of the Melnikov function, obtaining an asymptotic estimate for the maximal splitting distance, which is exponentially small in $\varepsilon$, and valid for all sufficiently small values of $\varepsilon$. This estimate behaves like $\exp \left\{-h_{1}(\varepsilon) / \varepsilon^{1 / 6}\right\}$ and we provide, for the first time in a system with 3 frequencies, an accurate description of the (positive) function $h_{1}(\varepsilon)$ in the numerator of the exponent, showing that it can be explicitly constructed from the resonance properties of the frequency vector $\omega$, and proving that it is a quasiperiodic function (and not periodic) with respect to $\ln \varepsilon$. In this way, we emphasize the strong dependence of the estimates for the splitting on the arithmetic properties of the frequencies.
\end{abstract}

\section{Introduction and Setup}

1.1. Background and state of the art. In nearly-integrable Hamiltonian systems with $n \geq 2$ degrees of freedom, irregular motion may take place near $(n-1)$-dimensional

This work has been partially supported by the Spanish MINECO/FEDER Grants MTM2015-65715, PGC2018-098676-B-I00 (the three authors) and MTM2016-80117-P (the author MG), the Catalan Grants 2017SGR1049 (AD and PG) and 2017SGR1374 (MG), the Russian Scientific Foundation Grant 14-41-00044 (AD and MG), and the Juan de la Cierva-Formación/Incorporación fellowships FJCI-2014-21229 and IJCI2016-29071 (MG).

\begin{tabular}{|l|l|l|l|l|l|}
\hline 220 & $\mathbf{3 8 3 2}$ & $\mathbf{B}$ & $\begin{array}{l}\text { Dispatch: 13/8/2020 } \\
\text { Total pages: 46 } \\
\text { Disk Received } \\
\text { Disk Used } \square\end{array}$ & $\begin{array}{l}\text { Journal: Commun. Math. Phys. } \\
\text { Not Used } \square \\
\text { Corrupted } \square \\
\text { Mismatch } \square\end{array}$ \\
\hline Jour. No & Ms. No.
\end{tabular}


whiskered tori (invariant hyperbolic tori) and their whiskers (invariant manifolds). In adequate scaled canonical coordinates (see for instance [DG01,Loc90,DGG14a] and references therein for more details about this introductory paragraph), these whiskered tori have frequency vectors with fast frequencies and their non-small hyperbolic part is typically given by a pendulum. The fundamental phenomenon guaranteeing irregular behavior near these whiskered tori is the non-coincidence of their whiskers, which is called the splitting of separatrices. The size of this splitting provides a measure of the irregular motion (and also of the global instability for $n \geq 3$ degrees of freedom) but is non-easily computable, since it turns out to be exponentially small with respect to the perturbation parameter. To worse things, for $n \geq 3$, the exponent in the splitting depends strongly on the arithmetic properties of the $(n-1)$-dimensional frequency vectors of the whiskered torus. Fortunately, for $n=3$ the standard theory of continued fractions can be successfully applied to the 2-dimensional frequency vectors of the whiskered tori to compute the splitting. Nevertheless, for $n \geq 4$ degrees of freedom, the standard theory of continued fractions cannot be applied to $(n-1)$-dimensional frequency vectors, and so far there are no computations of the exponentially small splitting of separatrices for whiskered tori with dimension greater or equal than three.

This paper is dedicated to the study and computation of the exponentially small splitting of separatrices, in a perturbed Hamiltonian system with 4 degrees of freedom, associated to a 3-dimensional whiskered torus with a cubic frequency vector. More precisely, we start with an integrable Hamiltonian $H_{0}$ possessing whiskered tori with a homoclinic whisker or separatrix, formed by coincident stable and unstable whiskers, and we focus our attention on a concrete torus with a frequency vector of fast frequencies:

$$
\omega_{\varepsilon}=\frac{\omega}{\sqrt{\varepsilon}}, \quad \omega=(1, \Omega, \widetilde{\Omega})
$$

with a small (positive) parameter $\varepsilon$, and we assume that the frequency ratios $\Omega=\omega_{2} / \omega_{1}$ and $\widetilde{\Omega}=\omega_{3} / \omega_{1}$ (it can be assumed that $\omega_{1}=1$ ) generate a complex cubic field (also called a non-totally real cubic field). This amounts to assume that $\Omega$ is a cubic irrational number (a real root of a polynomial of degree 3 with rational coefficients, that is not rational or quadratic) whose two conjugates are not real, and $\widetilde{\Omega}=a_{0}+a_{1} \Omega+a_{2} \Omega^{2}$, with $a_{0}, a_{1}, a_{2} \in \mathbb{Q}, a_{2} \neq 0$ (see Sect. 2.1 for more details). A paradigmatic example is the vector $\omega=\left(1, \Omega, \Omega^{2}\right)$, where $\Omega$ is the cubic golden number (the real number satisfying $\Omega^{3}=1-\Omega$, see Sect. 2.3$)$.

If we consider a perturbed Hamiltonian $H=H_{0}+\mu H_{1}$, where $\mu$ is small, in general the whiskers do not coincide anymore. This phenomenon has got the name of splitting of separatrices, which is related to the non-integrability of the system and the existence of chaotic dynamics, and plays a key role in the description of Arnold diffusion. If we assume, for the two involved parameters, a relation of the form $\mu=\varepsilon^{r}$ for some $r>0$, we have a problem of singular perturbation and in this case the splitting is exponentially small with respect to $\varepsilon$. Our aim is to provide an asymptotic estimate for the maximal splitting distance, and to show the dependence of such estimate on the arithmetic properties of the cubic number $\Omega$.

To provide a measure for the splitting, we can restrict ourselves to a transverse section to the unperturbed separatrix, and introduce the splitting function $\theta \in \mathbb{T}^{3} \mapsto \mathcal{M}(\theta) \in$ $\mathbb{R}^{3}$, providing the vector distance between the whiskers on this section, along the complementary directions. In this way, one obtains a measure for the maximal splitting distance as the maximum of the function $|\mathcal{M}(\theta)|$. On the other hand, in suitable coordinates the splitting function is the gradient of a scalar function called splitting potential [Eli94,DG00],

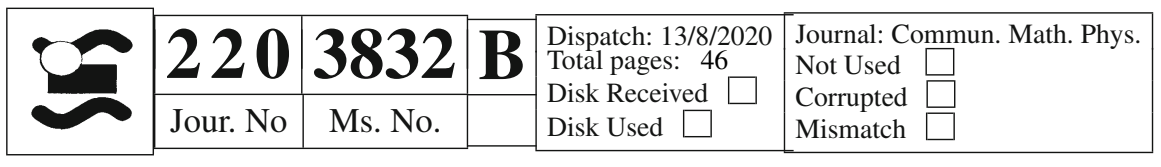




$$
\mathcal{M}(\theta)=\nabla \mathcal{L}(\theta),
$$

which implies that there always exist homoclinic orbits, which correspond to the zeros of $\mathcal{M}(\theta)$, i.e. the critical points of $\mathcal{L}(\theta)$.

In order to provide a first order approximation to the splitting function, with respect to the parameter $\mu$, it is very usual to apply the Poincaré-Melnikov method, introduced by Poincaré in his memoir [Poi90] and rediscovered much later by Melnikov and Arnold [Mel63, Arn64]. This method provides an approximation

$$
\mathcal{M}(\theta)=\mu M(\theta)+\mathcal{O}\left(\mu^{2}\right)
$$

given by the (vector) Melnikov function $M(\theta)$, defined by an integral (see for instance [Tre94,DG00]). As a result, one obtains asymptotic estimates for the maximum of the function $|\mathcal{M}(\theta)|$, provided $\mu$ is small enough. In fact, the Melnikov function can also be written as the gradient of a scalar function called the Melnikov potential: $M(\theta)=\nabla L(\theta)$.

However, the case of fast frequencies $\omega_{\varepsilon}$ as in (1), with a perturbation of order $\mu=\varepsilon^{r}$, for a given $r$ as small as possible, turns out to be, as said before, a singular problem. The difficulty comes from the fact that the Melnikov function $M(\theta)$ is exponentially small in $\varepsilon$, and the Poincaré-Melnikov method can be directly applied only if one assumes that $\mu$ is exponentially small with respect to $\varepsilon$ (see for instance [DG01] for more details). In order to validate the method in the case $\mu=\varepsilon^{r}$, one has to ensure that the error term is also exponentially small, and that the Poincaré-Melnikov approximation dominates it. To overcome such a difficulty in the study of the exponentially small splitting, Lazutkin introduced in [Laz03] the use of parameterizations of the whiskers on a complex strip (whose width is defined by the singularities of the unperturbed parameterized separatrix) by periodic analytic functions, together with flow-box coordinates. This tool was initially developed for the Chirikov standard map [Laz03], and allowed several authors to validate the Poincaré-Melnikov method for Hamiltonians with one and a half degrees of freedom (with only 1 frequency) [HMS88,Sch89,DS92,DS97,Ge197] and for area-preserving maps [DR98].

Later, those methods were extended to the case of whiskered tori with 2 frequencies: $\omega=(1, \Omega)$. In this case, the arithmetic properties of the frequencies play an important role in the exponentially small asymptotic estimates of the splitting function, due to the presence of small divisors of the form $k_{1}+k_{2} \Omega$ for integer numbers $k_{1}, k_{2}$. Such arithmetic properties can be carefully studied with the help of the standard theory of continued fractions. The role of the small divisors in the estimates of the splitting was first noticed by Lochak [Loc90] (who obtained an upper bound with an exponent coinciding with Nekhoroshev resonant normal forms [Nek77]), and also by Simó [Sim94] (generalizing an averaging procedure introduced in [Nei84]). Analogous estimates could also be obtained from a careful averaging out of the fast angular variables [Tre97,PT00], at least concerning sharp upper bounds of the splitting.

On the other hand, a numerical detection of asymptotic estimates was carried out in [Sim94], and they were rigorously proved in [DGJS97] for the quasiperiodically forced pendulum, assuming a polynomial perturbation in the coordinates associated to the pendulum. A more general (meromorphic) perturbation was considered in [GS12]. It is worth mentioning that, in some cases, the Poincaré-Melnikov method does not predict correctly the size of the splitting, as shown in [BFGS12], where a HamiltonJacobi method is instead used. This method had previously been used in [Sau01,LMS03, RW00, Bal06]. Similar asymptotic results were obtained in [DG04] for the concrete case of the famous golden ratio $\Omega=(\sqrt{5}-1) / 2$, and in [DGG14c] for the case of the silver

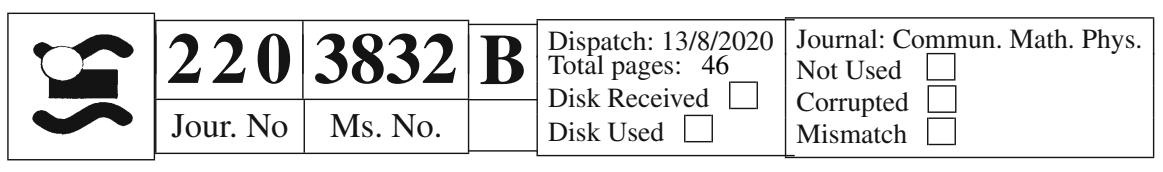


ratio $\Omega=\sqrt{2}-1$, and generalized in [DGG16] to any quadratic frequency ratio, and in [DGG14b] to any frequency ratio of constant type, i.e. with bounded partial quotients. Very recent results for frequency vectors with unbounded partial quotients can be found in the papers [FSV18a,FSV18b], which provide a heuristic analysis of the splitting.

In this paper, we consider a 3-dimensional torus with a frequency vector $\omega$ as in (1) whose ratios generate a complex cubic field (for short, we say a cubic vector "of complex type"). An important difference with respect to the 2-dimensional case is that in the 3dimensional case there is no standard theory of continued fractions allowing a simple analysis of the small divisors. As a paradigmatic example, we consider $\omega=\left(1, \Omega, \Omega^{2}\right)$ where $\Omega \approx 0.682328$ is the real number satisfying $\Omega^{3}=1-\Omega$, which has been called the cubic golden number (see for instance [HK00]). Other famous exemples have been considered in [Cha02] (see also [Loc92] for an account of examples and results concerning cubic frequencies).

Our goal is to develop a methodology, based on iteration matrices from a result by Koch [Koc99] (see Sect. 2.1) allowing us to study the resonances of the given cubic frequency vector. As a result, we obtain asymptotic estimates for the maximal splitting distance, whose dependence on $\varepsilon$ is described by a positive piecewise-smooth function denoted $h_{1}(\varepsilon)$ (see Theorem 1). In this paper it is proved for the first time that this function is quasiperiodic (and not periodic) with respect to $\ln \varepsilon$ with two frequencies $\alpha_{1}$ and $\alpha_{2}$, and its behavior depends strongly on the arithmetic properties of the cubic frequency vector $\omega$. In particular, we show that the function $h_{1}(\varepsilon)$ can be constructed explicitly from the study of the quasi-resonances of the frequency vector $\omega$, and we can also determine explicitly the frequencies $\alpha_{1}$ and $\alpha_{2}$, as well as upper and lower bounds for $h_{1}(\varepsilon)$. In this way, we provide an indication of the complexity of the dependence on $\varepsilon$ of the splitting.

Such results were partially established in the announcement [DGG14a] with a parallel study of the quadratic and cubic cases (with 2 and 3 frequencies, respectively), obtaining also exponentially small estimates for the maximal splitting distance, showing the periodicity of the function $h_{1}(\varepsilon)$ with respect to $\ln \varepsilon$ in the quadratic case (we also stress that this function becomes a constant in the case of only 1 frequency, see for instance [DS97]). Nevertheless, in [DGG14a] the quasiperiodicity of the function $h_{1}(\varepsilon)$ in the cubic case was only conjectured.

We point out that the aim of this paper is to obtain estimates for the maximal splitting distance, like in our paper [DGG14b] where we considered frequencies of constant type for a 2-dimensional torus. This is in constrast with most of the papers quoted in the previous paragraphs, which rather focus their attention on the transversality of the splitting. The study of the transversality could also be carried out with the methodology developed here, by means of a more accurate study, as done in [DG04,DGG14c, DGG16] for the quadratic case (see Remark 2(b)). We stress that, for some purposes, it is not necessary to establish the transversality of the splitting, and it can be enough to provide estimates of the maximal splitting distance. Indeed, such estimates imply the existence of splitting between the invariant manifolds, which provides a strong indication of the non-integrability of the system near the given torus, and opens the door to the application of topological methods [GR03, GL06] for the study of Arnold diffusion in such systems.

1.2. Setup. Here we describe the nearly-integrable Hamiltonian system under consideration. In particular, we study a singular or weakly hyperbolic (a priori stable) Hamiltonian with 4 degrees of freedom possessing a 3-dimensional whiskered torus with fast frequencies. In canonical coordinates $(x, y, \varphi, I) \in \mathbb{T} \times \mathbb{R} \times \mathbb{T}^{3} \times \mathbb{R}^{3}$, with the symplectic

$\therefore=$


form $\mathrm{d} x \wedge \mathrm{d} y+\mathrm{d} \varphi \wedge \mathrm{d} I$, the Hamiltonian is defined by

$$
\begin{aligned}
H(x, y, \varphi, I) & =H_{0}(x, y, I)+\mu H_{1}(x, \varphi), \\
H_{0}(x, y, I) & =\left\langle\omega_{\varepsilon}, I\right\rangle+\frac{1}{2}\langle\Lambda I, I\rangle+\frac{y^{2}}{2}+\cos x-1, \\
H_{1}(x, \varphi) & =h(x) f(\varphi) .
\end{aligned}
$$

Our system has two parameters $\varepsilon>0$ and $\mu$, linked by a relation $\mu=\varepsilon^{r}, r>0$ (the smaller $r$ the better). Thus, if we consider $\varepsilon$ as the unique parameter, we have a singular problem for $\varepsilon \rightarrow 0$. See [DG01] for a discussion about singular and regular problems.

Recall that we are assuming a vector of fast frequencies $\omega_{\varepsilon}=\omega / \sqrt{\varepsilon}$ with a cubic vector $\omega \in \mathbb{R}^{3}$ of "complex type", as introduced in (1). It is a well-known property (and we prove it in Sect. 2.2; see also [Cas57, Sect. V.3] or [Sch80, Sect. II.4]) that any (complex or totally real) cubic vector satisfies a Diophantine condition

$$
|\langle k, \omega\rangle| \geq \frac{\gamma}{|k|^{2}}, \quad \forall k \in \mathbb{Z}^{3} \backslash\{0\},
$$

with some $\gamma>0$ (the exponent 2 in this condition is the minimal one among vectors in $\left.\mathbb{R}^{3}\right)$. We also assume in (4) that $\Lambda$ is a symmetric $(3 \times 3)$-matrix, such that $H_{0}$ satisfies the condition of isoenergetic nondegeneracy

$$
\operatorname{det}\left(\begin{array}{cc}
\Lambda & \omega \\
\omega^{\top} & 0
\end{array}\right) \neq 0
$$

For the perturbation $H_{1}$ in (5), we deal with the following analytic periodic functions,

$$
h(x)=\cos x, \quad f(\varphi)=\sum_{k \in \mathcal{Z}} f_{k} \cos \left(\langle k, \varphi\rangle-\sigma_{k}\right), \quad \text { with } \quad f_{k}=\mathrm{e}^{-\rho|k|} \text { and } \sigma_{k} \in \mathbb{T}
$$

(we write the harmonics of Fourier expansions in the form of amplitude and phase) where we introduce, in order to avoid repetitions in the Fourier series, the set

$$
\mathcal{Z}=\left\{k \in \mathbb{Z}^{3}: k_{2} \geq 1 \text { or }\left(k_{2}=0, k_{3} \geq 1\right) \text { or }\left(k_{2}=k_{3}=0, k_{1} \geq 0\right)\right\},
$$

with $k=\left(k_{1}, k_{2}, k_{3}\right)$ (the specific choice of $k_{2}$ being positive, which is not relevant, allows us to agree with the definition of the set $\mathcal{P}$ in (44)). Notice that, for any couple $\pm k$ of integer vectors, only one of them belongs to $\mathcal{Z}$. The constant $\rho>0$ gives the complex width of analyticity of the function $f(\varphi)$. Concerning the phases $\sigma_{k}$, they can be chosen arbitrarily for the purpose of this paper.

To justify the form of the perturbation $H_{1}$ chosen in (5) and (9), we stress that it makes easier the explicit computation of the Melnikov potential, which is necessary in order to show that it dominates the error term in (3), and therefore to establish the existence of splitting. Moreover, the assumption that all coefficients $f_{k}$ in the Fourier expansion (9) with respect to $\varphi$ are nonzero and have an exponential decay, is usual in the literature (see for instance [FSV18a,FSV18b]), and ensures that the study of the dominant harmonics of the Melnikov potential can be carried out directly from the arithmetic properties of the frequency vector $\omega$. Indeed, such dominant harmonics correspond to the integer vectors $k$ providing an approximate equality in (7), i.e. giving the "smallest" divisors (relatively to the size of $|k|)$. We call primary resonances of $\omega$ to such vectors $k$, and secondary

\begin{tabular}{|l|l|l|l|l|l|}
\hline 220 & $\mathbf{3 8 3 2}$ & $\mathbf{B}$ & $\begin{array}{l}\text { Dispatch: 13/8/2020 } \\
\text { Total pages: 46 } \\
\text { Disk Received } \\
\text { Disk Used } \square\end{array}$ & $\begin{array}{l}\text { Journal: Commun. Math. Phys. } \\
\text { Not Used } \square \\
\text { Corrupted } \square \\
\text { Mismatch } \square\end{array}$ \\
\hline Jour. No & Ms. No.
\end{tabular}


resonances to the rest of quasi-resonances (see Sect. 2 for details). In this way, the choice of the coefficients $f_{k}$ in (9) allows us to emphasize the dependence of the splitting on the arithmetic properties of $\omega$.

It is worth remarking that, once we know the primary resonances for the given frequency vector $\omega$, we do not need all the coefficients $f_{k}$ to be different from zero in (9), but only the ones corresponding to primary resonances. On the other hand, since our method is completely constructive, other choices of concrete harmonics $f_{k}$ could also be considered (like $f_{k}=|k|^{m} \mathrm{e}^{-\rho|k|}$ ), simply at the cost of more cumbersome computations in order to determine the dominant harmonics of the Melnikov potential.

We also remind that the Hamiltonian defined in (4-9) is paradigmatic, since it is a generalization of the famous Arnold's example (introduced in [Arn64] to illustrate the transition chain mechanism in Arnold diffusion). It provides a model for the behavior of a nearly-integrable Hamiltonian system near a single resonance (see [DG01] for a motivation), and has often been considered in the literature (see for instance [GGM99, PT00,LMS03, DGS04]).

Let us describe the invariant tori and whiskers, as well as the splitting and Melnikov functions. First, it is clear that the unperturbed system given by $H_{0}$ (that corresponds to $\mu=0)$ is separable, and consists of the pendulum given by $P(x, y)=y^{2} / 2+\cos x-1$, and 3 rotors with fast frequencies: $\dot{\varphi}=\omega_{\varepsilon}+\Lambda I, \dot{I}=0$. The pendulum has a hyperbolic equilibrium at the origin, with separatrices that correspond to the curves given by $P(x, y)=0$. We parameterize the upper separatrix of the pendulum as $\left(x_{0}(s), y_{0}(s)\right)$, $s \in \mathbb{R}$, where

$$
x_{0}(s)=4 \arctan \mathrm{e}^{s}, \quad y_{0}(s)=\frac{2}{\cosh s} .
$$

Then, the lower separatrix has the parametrization $\left(x_{0}(-s),-y_{0}(-s)\right)$. For the rotors system $(\varphi, I)$, the solutions are $I=I_{0}, \varphi=\varphi_{0}+t\left(\omega_{\varepsilon}+\Lambda I_{0}\right)$. Consequently, the Hamiltonian $H_{0}$ has a 3-parameter family of 3-dimensional whiskered tori: in coordinates $(x, y, \varphi, I)$, each torus can be parameterized as

$$
\mathcal{T}_{I_{0}}: \quad\left(0,0, \theta, I_{0}\right), \quad \theta \in \mathbb{T}^{3},
$$

and the inner dynamics on each torus is $\dot{\theta}=\omega_{\varepsilon}+\Lambda I_{0}$. Each invariant torus has a homoclinic whisker, i.e. coincident 4-dimensional stable and unstable invariant manifolds, which can be parameterized as

$$
\mathcal{W}_{I_{0}}: \quad\left(x_{0}(s), y_{0}(s), \theta, I_{0}\right), \quad s \in \mathbb{R}, \theta \in \mathbb{T}^{3},
$$

with the inner dynamics given by $\dot{s}=1, \dot{\theta}=\omega_{\varepsilon}+\Lambda I_{0}$.

In fact, the collection of the whiskered tori for all values of $I_{0}$ is a 6-dimensional normally hyperbolic invariant manifold, parameterized by $(\theta, I) \in \mathbb{T}^{3} \times \mathbb{R}^{3}$. This manifold has a 7-dimensional homoclinic manifold, which can be parameterized by $(s, \theta, I)$, with inner dynamics $\dot{s}=1, \dot{\theta}=\omega_{\varepsilon}+\Lambda I, \dot{I}=0$ (see for instance [DLS06]).

Among the family of whiskered tori and homoclinic whiskers, we are going to focus our attention on the torus $\mathcal{T}_{0}$, whose frequency vector is $\omega_{\varepsilon}$ as in (1), and its associated homoclinic whisker $\mathcal{W}_{0}$.

When adding the perturbation $\mu H_{1}$, for $\mu \neq 0$ small enough the hyperbolic KAM theorem can be applied thanks to the Diophantine condition (7) and to the isoenergetic nondegeneracy (8). For $\mu$ small enough, the whiskered torus persists with some shift and

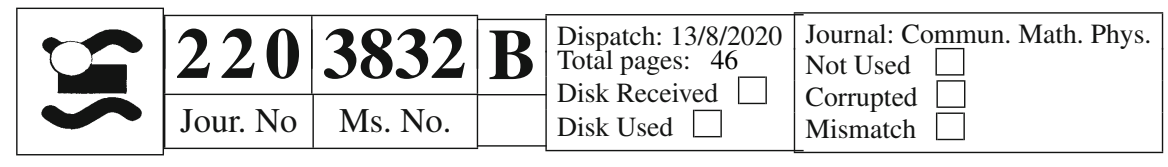


deformation, as a perturbed torus $\mathcal{T}=\mathcal{T}^{(\mu)}$, as well as its local whiskers $\mathcal{W}_{\text {loc }}=\mathcal{W}_{\text {loc }}^{(\mu)}$ (precise statements can be found, for instance, in [Nie00,DGS04]).

The local whiskers can be extended along the flow, but in general for $\mu \neq 0$ the (global) whiskers do not coincide anymore, and one expects the existence of splitting between the (4-dimensional) stable and unstable whiskers, denoted $\mathcal{W}^{\mathrm{s}}=\mathcal{W}^{\mathrm{s},(\mu)}$ and $\mathcal{W}^{\mathrm{u}}=\mathcal{W}^{\mathrm{u},(\mu)}$ respectively. Using flow-box coordinates (see [DGS04], where the $n$ dimensional case is considered) in a neighbourhood containing a piece of both whiskers (away from the invariant torus), one can introduce parameterizations of the perturbed whiskers, with parameters $(s, \theta)$ inherited from the unperturbed whisker (12), and the inner dynamics

$$
\dot{s}=1, \quad \dot{\theta}=\omega_{\varepsilon} .
$$

Then, the distance between the stable whisker $\mathcal{W}^{\mathrm{s}}$ and the unstable whisker $\mathcal{W}^{\mathrm{u}}$ can be measured by comparing such parameterizations along the complementary directions. The number of such directions is 4 but, due to the energy conservation, it is enough to consider 3 directions, say the ones related to the action coordinates $I$.

In order to measure correctly the splitting between the invariant manifolds $\mathcal{W}^{\mathrm{s}}$ and $\mathcal{W}^{\mathrm{u}}$, their parameterizations should be chosen in a coordinated way. For example, this can be done with the help of a near-identity exact symplectic map as in [DG00, Sect. 5.1] (following an idea introduced in [Eli94]). This map takes a piece of $\mathcal{W}^{\mathrm{s}}$ into $\mathcal{W}^{\mathrm{u}}$, which allows one to relate the parameterizations of both whiskers. With an additional change of parameters, the unstable whisker $\mathcal{W}^{\mathrm{u}}$ appears as a graph over the stable whisker $\mathcal{W}^{\mathrm{s}}$ and, by the properties of the whiskers as Lagrangian manifolds, the conjugate coordinates (energy and actions) become a gradient of a scalar function. In the case of fast frequencies, the distance is shown to be exponentially small with respect to $\varepsilon$ in [DGS04].

In this way, one can introduce a (vector) splitting function, with values in $\mathbb{R}^{3}$, as the difference of the parameterizations $\mathcal{J}^{\mathrm{s}, \mathrm{u}}(s, \theta)$ of (the action components of) the perturbed whiskers $\mathcal{W}^{\mathrm{s}}$ and $\mathcal{W}^{\mathrm{u}}$. Initially this function depends on $(s, \theta)$,

$$
\widetilde{\mathcal{M}}(s, \theta):=\mathcal{J}^{\mathrm{u}}(s, \theta)-\mathcal{J}^{\mathrm{s}}(s, \theta), \quad|s| \leq \kappa, \theta \in \mathbb{T}^{3},
$$

with $\kappa$ providing an interval where both parameterizations can be defined and hence compared. Thanks to the use of flow-box coordinates, the function $\widetilde{\mathcal{M}}$ turns out to be $\omega_{\varepsilon}$-quasiperiodic (see [DGS04]):

$$
\widetilde{\mathcal{M}}(s, \theta)=\widetilde{\mathcal{M}}\left(0, \theta-\omega_{\varepsilon} s\right) .
$$

On the other hand, the funcion $\widetilde{\mathcal{M}}$ can be extended to a suitable complex strip in the variables $(s, \theta)$. This fact and the quasiperiodicity play a key role in order to obtain exponentially small estimates (see Sect. 4, where we apply the results of [DGS04]). In fact, we may consider the restriction to a fixed $s$ providing a transverse section, say $s=0$ (which lies close to $x=\pi$ by (11)), and we define as in [DG00, Sect. 5.2] our splitting function as

$$
\mathcal{M}(\theta):=\widetilde{\mathcal{M}}(0, \theta), \quad \theta \in \mathbb{T}^{3},
$$

and we refer to (13) as the "full" splitting funcion. We point out, as an alternative approach, that a splitting function can also be defined by considering parametrizations of the whiskers as solutions of Hamilton-Jacobi equation (see for instance [LMS03, BFGS12]).

\begin{tabular}{|l|l|l|l|l|l|}
\hline $\mathbf{2} 2 \mathbf{2 0}$ & $\mathbf{3 8 3 2}$ & $\mathbf{B}$ & $\begin{array}{l}\text { Dispatch: 13/8/2020 } \\
\text { Total pages: 46 } \\
\text { Disk Received } \\
\text { Disk Used } \square\end{array}$ & $\begin{array}{l}\text { Journal: Commun. Math. Phys. } \\
\text { Not Used } \square \\
\text { Corrupted } \\
\text { Mismatch } \square\end{array}$ \\
\hline Jour. No & Ms. No.
\end{tabular}


Applying the Poincaré-Melnikov method, the first order approximation (3) of the splitting function is given by the (vector) Melnikov function $M(\theta)$, which is the gradient of the (scalar) Melnikov potential: $M(\theta)=\nabla L(\theta)$. The latter one can be defined as an integral: we consider any homoclinic trajectory of the unperturbed homoclinic whisker $\mathcal{W}_{0}$ in (12), starting on the section $s=0$, and the trajectory on the torus $\mathcal{T}_{0}$ to which it is asymptotic as $t \rightarrow \pm \infty$, and we substract the values of the perturbation $H_{1}$ on the two trajectories. This gives an absolutely convergent integral, which depends on the initial phase $\theta \in \mathbb{T}^{3}$ of the considered trajectories:

$$
\begin{aligned}
L(\theta) & :=-\int_{-\infty}^{\infty}\left[H_{1}\left(x_{0}(t), \theta+t \omega_{\varepsilon}\right)-H_{1}\left(0, \theta+t \omega_{\varepsilon}\right)\right] \mathrm{d} t \\
& =-\int_{-\infty}^{\infty}\left[h\left(x_{0}(t)\right)-h(0)\right] f\left(\theta+t \omega_{\varepsilon}\right) \mathrm{d} t,
\end{aligned}
$$

where we have taken into account the specific form (5) of the perturbation.

Our choices of the pendulum $P(x, y)=y^{2} / 2+\cos x-1$ in (4) and the perturbation in (5) and (9) lead to simple poles in the integrand in (16), which makes it possible to use the method of residues in order to compute the coefficients $L_{k}$ of the Fourier expansion of the Melnikov potential $L(\theta)$, and hence the (vector) coefficients $M_{k}$ of the Melnikov function, which satisfy $\left|M_{k}\right|=|k|\left|L_{k}\right|$. Such coefficients turn out to be exponentially small in $\varepsilon$ (see their expression in Sect. 3.1). For each value of $\varepsilon$ only the dominant harmonic, corresponding to some index $k=S_{1}(\varepsilon)$, is relevant in order to provide asymptotic estimates for the maximum value of the Melnikov function (of course, a few dominant harmonics may have to be considered near some transition values of $\varepsilon$, at which changes in the dominance take place). Due to the exponential decay of the Fourier coefficients of $f(\varphi)$ in (9), it is not hard to study such a dominance and its dependence on $\varepsilon$.

In order to give asymptotic estimates for the maximal splitting distance, the estimates obtained for the Melnikov function $M(\theta)$ have to be validated also for the splitting function $\mathcal{M}(\theta)$. The difficulty in the application of the Poincaré-Melnikov approximation (3), due to the exponential smallness in $\varepsilon$ of the function $M(\theta)$ in our singular case $\mu=\varepsilon^{r}$, can be solved by obtaining upper bounds (on a complex domain) for the error term in (3), showing that, if $r>r^{*}$ with a suitable $r^{*}$, its Fourier coefficients are dominated by the coefficients of $M(\theta)$ (see also [DGS04]).

1.3. Main result. For the Hamiltonian system (4-9) with the 2 parameters linked by $\mu=\varepsilon^{r}, r>r^{*}$ (with some suitable $r^{*}$ ), and a cubic frequency vector of complex type $\omega$ as in (1), our main result provides an exponentially small asymptotic estimate for the maximal distance of splitting, given in terms of the maximum size in modulus of the splitting function $\mathcal{M}(\theta)$, and this estimate is valid for all $\varepsilon$ sufficiently small.

With our approach, the Poincaré-Melnikov method can be validated for an exponent $r>r^{*}$ with $r^{*}=3$, although a lower value of $r^{*}$ can be given in some particular cases (see Remark 2(c)). However, such values of $r^{*}$ are not optimal and could be improved using other methods, like the parametrization of the whiskers as solutions of HamiltonJacobi equation (see for instance [LMS03,BFGS12]). In this paper, the emphasis is put on the extension of the methods and results from the 2-dimensional quadratic case to the 3-dimensional cubic case, rather than on the improvement of the value of $r^{*}$.

Due to the form of $f(\varphi)$ in (9), the Melnikov potential $L(\theta)$ is readily presented in its Fourier series (see Sect. 3.1), with coefficients $L_{k}=L_{k}(\varepsilon)$ which are exponentially

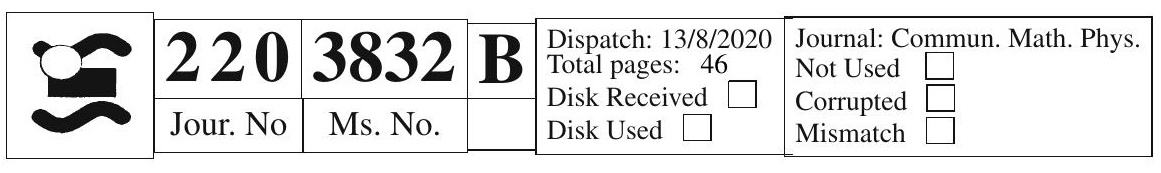


small in $\varepsilon$. We use this expansion of $L(\theta)$ in order to detect its dominant harmonic $k=S_{1}(\varepsilon)$ for every given $\varepsilon$. Such a dominance is also valid for the Melnikov function $M(\theta)$, since the size of their Fourier coefficients $M_{k}$ (vector) and $L_{k}$ (scalar) is directly related: $\left|M_{k}\right|=|k|\left|L_{k}\right|, k \in \mathcal{Z}$ (recall the definition of $\mathcal{Z}$ in (10)).

As shown in Sect. 4, in order to obtain an asymptotic estimate for the maximum value of $\mathcal{M}(\theta)$, i.e. for the distance of splitting, for most values of $\varepsilon$ it is enough to consider the (unique) first dominant harmonic $S_{1}(\varepsilon)$ of the Melnikov function $M(\theta)$, whose size behaves like $\exp \left\{-h_{1}(\varepsilon) / \varepsilon^{1 / 6}\right\}$, being described by a (positive) function $h_{1}(\varepsilon)$ that is carefully studied in this paper. To ensure that the dominant harmonic of $M(\theta)$ corresponds to the dominant harmonic of the splitting function $\mathcal{M}(\theta)$, one has to carry out an accurate control of the error term in (3). In this way, using estimates for the size of the dominant harmonic, as well as for all the remaining harmonics, one can prove that the dominant harmonic is large enough and provides an approximation to the maximum size of the whole splitting function (see also [DGG14a, DGG14b, DGG16]).

However, one has to consider at least two harmonics for $\varepsilon$ near to some "transition values", at which a change in the dominant harmonic occurs and, consequently, two (or more) harmonics having similar sizes can be considered as the dominant ones. In this case, the size of the splitting function can also be determined from the dominant harmonics, although such transition values turn out to be corners of the function $h_{1}(\varepsilon)$ (see the theorem below, and Fig. 1).

The determination of the dominant harmonics, and hence the dependence on $\varepsilon$ of the size of the splitting and the function $h_{1}(\varepsilon)$, are closely related to the arithmetic properties of the frequency vector $\omega$ in (1), since the integer vectors $k \in \mathcal{Z}$ associated to the dominant harmonics can be found, for any $\varepsilon$, among the main quasi-resonances of $\omega$, i.e. the vectors $k$ giving the "smallest" divisors $|\langle k, \omega\rangle|$ (relatively to the size of $|k|$ ). In Sect. 2, we develop a methodology for a complete study of the resonant properties of cubic frequency vectors (of complex type), which is one of the main goals of this paper. This methodology relies on the classification of the integer vectors $k$ into "resonant sequences" (see Sect. 2.1 for definitions). Among them, the sequence of primary resonances corresponds to the vectors $k$ which fit best the Diophantine condition (7), and the vectors $k$ belonging to the remaining sequences are called secondary resonances. In this way, we can also determine the (positive) asymptotic Diophantine constant,

$$
\gamma^{-}:=\liminf _{|k| \rightarrow \infty}|\langle k, \omega\rangle| \cdot|k|^{2} .
$$

This approach, already announced in [DGG14a] for 3-dimensional cubic frequency vectors, generalizes the one introduced in [DG03] for 2-dimensional quadratic frequency vectors.

For most values of $\varepsilon$, the dominant harmonic is given by an integer vector $k$ associated to a primary resonance, but for some intervals of $\varepsilon$ the secondary resonances may have to be taken into account giving rise to a more involved function $h_{1}(\varepsilon)$. Nevertheless, for some cubic frequency vectors $\omega$ in (1) such as the cubic golden vector, the function $h_{1}(\varepsilon)$ can be defined using only the primary resonances (see Sects. 2.3 and 3.4).

In order to generate the resonant sequences, we use a result by Koch [Koc99], ensuring the existence of a unimodular $(3 \times 3)$-matrix $T$ (i.e. with integer entries and determinant \pm 1 ), having $\omega$ as an eigenvector with the associated eigenvalue

$$
\lambda>1 \text {. }
$$

Altough there exist an infinity of matrices $T$ fitting Koch's result, we establish in Sect. 2.1 a canonical choice for it (see Proposition 4), and we write it as $T=T(\omega)$.

\begin{tabular}{|l|l|l|l|l|l|}
\hline 220 & $\mathbf{3 8 3 2}$ & $\mathbf{B}$ & $\begin{array}{l}\text { Dispatch: 13/8/2020 } \\
\text { Total pages: 46 } \\
\text { Disk Received } \\
\text { Disk Used } \square\end{array}$ & $\begin{array}{l}\text { Journal: Commun. Math. Phys. } \\
\text { Not Used } \square \\
\text { Corrupted } \square \\
\text { Mismatch } \square\end{array}$ \\
\hline Jour. No & Ms. No.
\end{tabular}



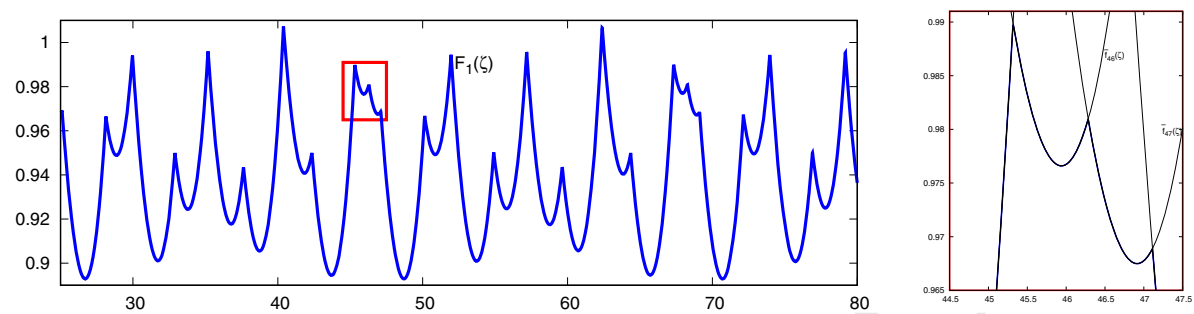

Fig. 1. Graph of the function $h_{1}(\varepsilon)=F_{1}(\zeta)$ in the exponent of (20), for the cubic golden vector (see Sect. 2.3), in the logarithmic variable $\zeta \sim \ln (1 / \varepsilon)$ (see (81) for a precise definition), as the minimum of the functions $\bar{f}_{n}(\zeta)$ (see Sects. 3.2 and 3.4)

The eigenvalue $\lambda=\lambda(\omega)$ is also a cubic irrational number and belongs to $\mathbb{Q}(\Omega)$. Hence it also has complex conjugates, which can be written in the form

$$
\lambda_{2}, \bar{\lambda}_{2}=\frac{1}{\sqrt{\lambda}} \mathrm{e}^{ \pm \mathrm{i} \pi \cdot \phi}, \quad 0<\phi<1,
$$

and $\phi=\phi(\omega)$ is an irrational number (see Sect. 2.1).

For a concrete cubic frequency vector $\omega$, it is not too hard to find the Koch's matrix $T=T(\omega)$ (see Sect. 2.1 for a procedure, and Sect. 2.3 for its application to the concrete case of the cubic golden vector). We point out that, for the quadratic 2-dimensional case $\omega=(1, \Omega)$, a systematic algorithm providing an analogous $(2 \times 2)$-matrix $T$ was developed in [DGG16], from the continued fraction of the frequency ratio $\Omega$ (which is eventually periodic for quadratic numbers). An extension of this algorithm to the cubic case would require a further study (possibly using some of the existing multidimensional continued fraction theories), and is not carried out here.

Assuming that the matrix $T$ is known, the key point is that the iteration of the matrix $U=\left(T^{-1}\right)^{\top}$ from an initial ("primitive") vector allows us to generate any resonant sequence (see the definition (45)). In this way, we can construct the resonant sequences allowing us to detect the dominant harmonics of the Melnikov potential and, consequently, asymptotic estimates for the maximal splitting distance.

Next, we establish the main result of this work, which generalizes to the complex cubic case the results obtained in [DG04,DGG16] for the quadratic case. The result given below provides exponentially small asymptotic estimates for the maximal distance of splitting, as $\varepsilon \rightarrow 0$, given by the maximum of $|\mathcal{M}(\theta)|, \theta \in \mathbb{T}^{3}$. In such asymptotic estimates, the dependence on $\varepsilon$ is mainly described by the exponent $1 / 6$, and by the function $h_{1}(\varepsilon)$. This is a positive function, quasiperiodic with respect to $\ln \varepsilon$ and piecewise-smooth and, consequently, it has a finite number of corners (i.e. jump discontinuities of the derivative) in any given interval. As we can see from the statement of the theorem, the numbers $\lambda$ and $\phi$ introduced in (18-19) play an essential role in the quasiperiodicity of the function $h_{1}(\varepsilon)$, since they provide directly the two frequencies $3 \ln \lambda$ and $3 \ln \lambda \cdot \phi$, and the fact that $\phi$ is irrational ensures that the function $h_{1}(\varepsilon)$ is not periodic, which makes a difference with respect to the quadratic case considered in [DGG16].

For any given cubic vector $\omega$ (of complex type), the function $h_{1}(\varepsilon)$ can be explicitly constructed (see Sect. 3.2). However, its (piecewise) expression can be very complicated. Its graph is shown in Fig. 1 (where a logarithmic scale for $\varepsilon$ is used), for the concrete case of the cubic golden frequency vector. The oscillatory behavior of the function $h_{1}(\varepsilon)$ depends strongly on the arithmetic properties of $\omega$.

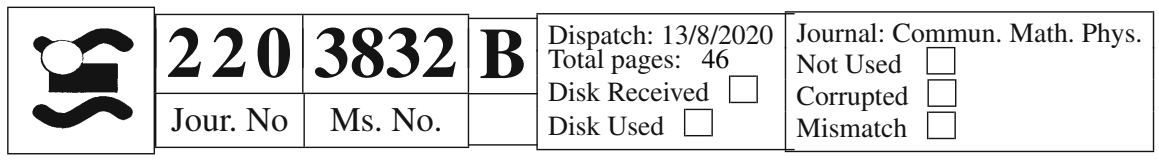


For positive quantities, we use the notation $f \sim g$ if we can bound $c_{1} g \leq f \leq c_{2} g$ with constants $c_{1}, c_{2}>0$ not depending on $\varepsilon, \mu$.

Theorem 1. (main result) Assume the conditions described for the Hamiltonian (4-9), with a cubic frequency vector $\omega=(1, \Omega, \widetilde{\Omega})$ of complex type as in (1), that $\varepsilon$ is small enough and that $\mu=\varepsilon^{r}, r>3$. Then, for the splitting function $\mathcal{M}(\theta)$ we have:

$$
\max _{\theta \in \mathbb{T}^{3}}|\mathcal{M}(\theta)| \sim \frac{\mu}{\varepsilon^{1 / 3}} \exp \left\{-\frac{C_{0} h_{1}(\varepsilon)}{\varepsilon^{1 / 6}}\right\} .
$$

The function $h_{1}(\varepsilon)$, defined in (87), is positive, piecewise-smooth, piecewise-convex and quasiperiodic in $\ln \varepsilon$, with two frequencies $3 \ln \lambda$ and $3 \ln \lambda \cdot \phi$, where $\lambda=\lambda(\omega)$ and $\phi=\phi(\omega)$ are the numbers introduced in (18-19). It satisfies for $\varepsilon>0$ lower and upper bounds $J_{0}^{-} \leq h_{1}(\varepsilon) \leq J_{1}^{+}$, where the values $J_{0}^{-}=J_{0}^{-}(\omega)$ and $J_{1}^{+}=J_{1}^{+}(\omega)$ are defined in (99). On the other hand, $C_{0}=C_{0}(\omega, \rho)$ is a positive constant defined in (74).

Remarks 2. (a) As a consequence of this theorem, replacing $h_{1}(\varepsilon)$ by its supremum value $J_{1}^{*}\left(\leq J_{1}^{+}\right.$, see also Sect. 3.3), we get the following sharp lower bound for the maximal splitting distance:

$$
\max _{\theta \in \mathbb{T}^{3}}|\mathcal{M}(\theta)| \geq \frac{c \mu}{\varepsilon^{1 / 3}} \exp \left\{-\frac{C_{0} J_{1}^{*}}{\varepsilon^{1 / 6}}\right\},
$$

where $c$ is a constant. This may be enough, if our aim is only to prove the existence of splitting of separatrices, without giving an accurate description for it.

(b) Our approach can also be applied to show the existence of transverse homoclinic orbits, associated to simple zeros $\theta^{*}$ of the splitting function $\mathcal{M}(\theta)$ (or, equivalently, nondegenerate critical points of the splitting potential), providing an asymptotic estimate for the transversality of the homoclinic orbits, measured by the minimum eigenvalue (in modulus) of the matrix $\operatorname{DM}\left(\theta^{*}\right)$ at each zero of $\mathcal{M}(\theta)$. Such an asymptotic estimate is exponentially small in $\varepsilon$ as in (20), but the function $h_{1}(\varepsilon)$ has to be replaced by a greater function $h_{3}(\varepsilon)$, also piecewise-smooth and quasiperiodic in $\ln \varepsilon$. In order to define $h_{3}(\varepsilon)$, one has to consider the three most dominant harmonics whose indices $S_{1}(\varepsilon), S_{2}(\varepsilon), S_{3}(\varepsilon) \in \mathcal{Z}$ are linearly independent (this is necessary in order to prove that the zeros $\theta^{*}$ are simple). This result on transversality would be valid for "almost all" $\varepsilon$ sufficiently small, since one has to exclude a small neighborhood of some values where the third and the fourth dominant harmonics have similar sizes, and homoclinic bifurcations could take place. See [DGG16] for the analogous situation in the quadratic case, where only the two most dominant harmonics are necessary.

(c) The results of Theorem 1 can be improved under some particular situations. For instance, if the function $h(x)$ in (9) is replaced by $h(x)=\cos x-1$, then the estimates are valid for $\mu=\varepsilon^{r}$ with $r>2$ (instead of $r>3$ ). The details of this improvement are not given here, since they work exactly as in [DG04].

Organization of the paper. We start in Sect. 2 with studying the arithmetic properties of cubic frequency vectors $\omega=(1, \Omega, \widetilde{\Omega}$ ) (of complex type), and constructing the iteration matrix $T$. Next, in Sect. 3 we find an asymptotic estimate for the dominant harmonic of the splitting potential, which allows us to define the function $h_{1}(\varepsilon)$ and study their general properties. In order to illustrate our methods, concrete results for

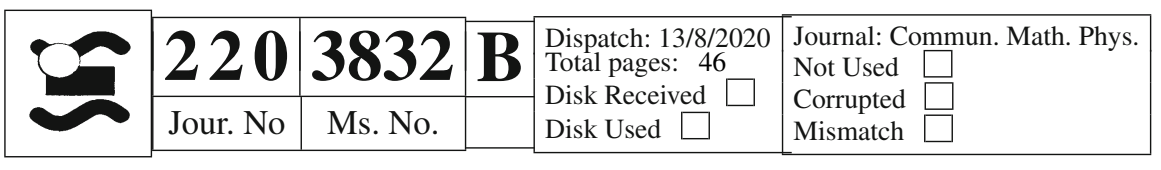


the cubic golden vector are obtained in Sects. 2.3 (arithmetic properties) and 3.4 (the function $h_{1}(\varepsilon)$ ). Finally, in Sect. 4 we provide rigorous bounds of the remaining harmonics allowing us to obtain asymptotic estimates for the maximal splitting distance, as established in Theorem 1.

\section{Arithmetic Properties of Cubic Frequencies}

2.1. Iteration matrix for a cubic frequency vector. We consider a cubic frequency vector $\omega \in \mathbb{R}^{3}$, i.e. the frequency ratios $\omega_{2} / \omega_{1}$ and $\omega_{3} / \omega_{1}$ generate a cubic field (an algebraic number field of degree 3 over $\mathbb{Q}$, i.e. its dimension as a vector space over $\mathbb{Q}$ is 3 ). In order to simplify our exposition, we assume that $\omega_{1}=1$, and hence the vector has the form

$$
\omega=(1, \Omega, \widetilde{\Omega}),
$$

where $\Omega$ is a cubic irrational number, i.e. its minimum polynomial (the monic polynomial of minimal degree having $\Omega$ as a root) has degree 3 , and $\widetilde{\Omega}$ belongs to the field $\mathbb{Q}(\Omega)$ :

$$
\begin{aligned}
\Omega^{3} & =r_{0}+r_{1} \Omega+r_{2} \Omega^{2}, \\
\widetilde{\Omega} & =a_{0}+a_{1} \Omega+a_{2} \Omega^{2}, \quad \text { with } a_{2} \neq 0,
\end{aligned}
$$

where the coefficients $r_{j}, a_{j}$ are rational. The number $\widetilde{\Omega}$ is also cubic irrational (in fact, any number belonging to $\mathbb{Q}(\Omega)$ is either rational or cubic irrational). We restrict ourselves to the complex case (also called the non-totally real case): the two conjugates of $\Omega$, as a root of the polynomial Eq. (22), are complex. This condition can be expressed in terms of having negative discriminant,

$$
\Delta=4 r_{1}^{3}+r_{1}^{2} r_{2}^{2}-27 r_{0}^{2}-18 r_{0} r_{1} r_{2}-4 r_{0} r_{2}^{3}<0 .
$$

We denote the conjugates of $\Omega$ as

$$
\Omega_{2}:=\sigma(\Omega)=\sigma_{2}+\mathrm{i} \sigma_{3}, \quad \bar{\Omega}_{2}=\bar{\sigma}(\Omega)=\sigma_{2}-\mathrm{i} \sigma_{3}
$$

and, from the standard equalities

$r_{2}=\Omega+\Omega_{2}+\bar{\Omega}_{2}=\Omega+2 \sigma_{2}, \quad r_{1}=-\left(\Omega \Omega_{2}+\Omega \bar{\Omega}_{2}+\Omega_{2} \bar{\Omega}_{2}\right)=-\left(2 \Omega \sigma_{2}+\sigma_{2}^{2}+\sigma_{3}^{2}\right)$

we see that

$$
\sigma_{2}=\frac{1}{2}\left(r_{2}-\Omega\right), \quad \sigma_{3}=\frac{s}{2} \sqrt{-\left(4 r_{1}+r_{2}^{2}\right)-2 r_{2} \Omega+3 \Omega^{2}},
$$

with a concrete sign $s= \pm 1$ for $\sigma_{3}$, that will be chosen later for convenience (see (37)).

It is clear from (23) that our cubic frequency vector $\omega$ can be related to the more particular case

$$
\omega^{(0)}=\left(1, \Omega, \Omega^{2}\right)
$$

through a linear change: $\omega=A \omega^{(0)}$, with the following matrix belonging to the general linear group $\mathrm{GL}(3, \mathbb{Q})$,

$$
A:=\left(\begin{array}{ccc}
1 & 0 & 0 \\
0 & 1 & 0 \\
a_{0} & a_{1} & a_{2}
\end{array}\right)
$$

(for instance, the cubic golden frequency vector considered in Sect. 2.3 has the form (26)).

\begin{tabular}{|l|l|l|l|l|l|}
\hline $\mathbf{2 0 0} 20$ & $\mathbf{3 8 3 2}$ & $\mathbf{B}$ & $\begin{array}{l}\text { Dispatch: 13/8/2020 } \\
\text { Total pages: 46 } \\
\text { Disk Received } \square \\
\text { Disk Used } \square\end{array}$ & $\begin{array}{l}\text { Journal: Commun. Math. Phys. } \\
\text { Not Used } \square \\
\text { Corrupted } \square \\
\text { Mismatch } \square\end{array}$ \\
\hline
\end{tabular}


It is well-known from algebraic number theory (see for instance [ST87, Ch. II] or [Lan02, Ch. V-VI] as general references) that there exist unique field isomorphisms $\sigma: \mathbb{Q}(\Omega) \longrightarrow \mathbb{Q}\left(\Omega_{2}\right)$ and $\bar{\sigma}: \mathbb{Q}(\Omega) \longrightarrow \mathbb{Q}\left(\bar{\Omega}_{2}\right)$ such that $\sigma(\Omega)=\Omega_{2}$ and $\bar{\sigma}(\Omega)=\bar{\Omega}_{2}$. It is clear that $\sigma$ and $\bar{\sigma}$ are related by the ordinary complex conjugacy. Then, the numbers $\sigma(\widetilde{\Omega})$ and $\bar{\sigma}(\widetilde{\Omega})$ turn to be the conjugates of $\widetilde{\Omega}$, and they are also complex (indeed, if they were real, they would coincide and $\widetilde{\Omega}$ would not be a cubic irrational).

Any cubic frequency vector $\omega \in \mathbb{R}^{3}$ satisfies a Diophantine condition, with the minimal exponent (see for instance [Cas57, Sect. V.3] or [Sch80, Sect. II.4]):

$$
|\langle k, \omega\rangle| \geq \frac{\gamma}{|k|^{2}}, \quad \forall k \in \mathbb{Z}^{3} \backslash\{0\} .
$$

With this in mind, we define the "numerators"

$$
\gamma_{k}:=|\langle k, \omega\rangle| \cdot|k|^{2}, \quad k \in \mathbb{Z}^{3} \backslash\{0\},
$$

where we use the Euclidean norm: $|\cdot|=|\cdot|_{2}$ (this allows us to use the properties of the scalar product). The numerators have $\gamma>0$ as a lower bound. Our goal is to provide a classification of the integer vectors $k$, according to the size of $\gamma_{k}$, in order to find the primary resonances (i.e. the integer vectors $k$ for which $\gamma_{k}$ is smallest, and hence best fitting the Diophantine condition (28)), and study their separation with respect to the remaining vectors $k$ (i.e. the secondary resonances).

The key point will be to use the following result by Koch [Koc99]: for a vector $\omega \in \mathbb{R}^{\ell}$ whose frequency ratios generate an algebraic field of degree $\ell$, there exists a unimodular $(\ell \times \ell$ )-matrix $T$ (a square matrix with integer entries and determinant \pm 1 ) having $\omega$ as an eigenvector with associated eigenvalue $\lambda$ of modulus $>1$, and such that the other $\ell-1$ eigenvalues are all simple and of modulus $<1$. This result is valid for any dimension $\ell$, and is usually applied in the context of renormalization theory (see for instance [Koc99,Lop02]), since the iteration of the matrix $T$ provides successive rational approximations to the direction of the vector $\omega$.

For any given cubic frequency vector $\omega$ as in (21), we say that a $(3 \times 3)$-matrix $T$ is a "Koch's matrix for $\omega$ " if it satisfies the requirements of Koch's result [Koc99]. It is not hard to find a Koch's matrix for any concrete cubic vector $\omega$ (see below for a general procedure, and Sect. 2.3 for its application to the concrete case of the cubic golden vector). It is clear that a Koch's matrix $T$ is not unique, since any power $\pm T^{n}$ is also a Koch's matrix.

We can assume that the determinant of $T$ is positive, $\operatorname{det} T=1$, i.e. $T$ belonging to the special linear group $\mathrm{SL}(3, \mathbb{Z})$ (otherwise, we can replace $T$ by $-T$ ). For the eigenvalue $\lambda$ associated to the eigenvector $\omega$, it is clear that it is real and can be writen as

$$
\lambda=\left\langle T_{(1)}, \omega\right\rangle=T_{11}+T_{12} \Omega+T_{13} \widetilde{\Omega} \in \mathbb{Q}(\Omega)
$$

where we denote $T_{(1)}:=\left(T_{11}, T_{12}, T_{13}\right)$ (the first row of $T$, considered here as a column vector). We also see that $\lambda$ is cubic irrational (otherwise, it would be rational and the frequency ratios of $\omega$ would also be rational). The other two eigenvalues of $T$, which are the conjugates of $\lambda$, are complex (see the argument given above for $\widetilde{\Omega}$ ), which implies that $\lambda$ is positive: $\lambda>1$. We write the conjugates of $\lambda$ in terms of real and imaginary parts:

$$
\lambda_{2}:=\sigma(\lambda)=\mu_{2}+\mathrm{i} \mu_{3}, \quad \bar{\lambda}_{2}=\bar{\sigma}(\lambda)=\mu_{2}-\mathrm{i} \mu_{3} .
$$

Moreover, we consider a basis of eigenvectors of $T$, also writing the two complex ones in terms of real and imaginary parts (thus, we do not work directly with complex vectors):

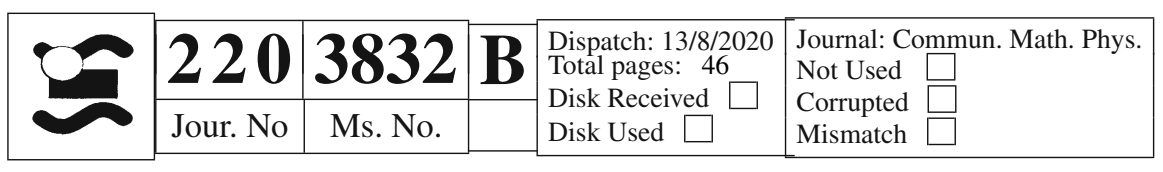




$$
\omega, \quad v_{2}+\mathrm{i} v_{3}=\sigma(\omega), \quad v_{2}-\mathrm{i} v_{3}=\bar{\sigma}(\omega),
$$

with associated eigenvalues $\lambda, \lambda_{2}, \bar{\lambda}_{2}$, respectively. We understand that, for vectors, the conjugacies $\sigma, \bar{\sigma}$ can be applied componentwisely, and hence the conjugate vectors above can be obtained just by replacing $\Omega$ by $\Omega_{2}$ or $\bar{\Omega}_{2}$ in (21). In this way, the vectors $v_{2}$ and $v_{3}$ do not depend on the specific choice of a Koch's matrix $T$. Let $C$ denote the $(3 \times 3)$-matrix having $\omega, v_{2}, v_{3}$ as columns, and we consider its condition number

$$
\kappa=\kappa(\omega):=|C| \cdot\left|C^{-1}\right|
$$

also not depending on the choice of $T$ (we use the matrix norm subordinate to the Euclidean norm for vectors). Next, we prove that the eigenvalue $\lambda>1$ cannot be arbitrarily close to 1 .

Lemma 3. For any Koch's matrix $T \in \mathrm{SL}(3, \mathbb{Z})$ for $\omega$, the real eigenvalue $\lambda$ in (30) satifies the lower bound $\lambda>\lambda_{0}$, with $\lambda_{0}=\lambda_{0}(\omega)>1$ defined as the unique real number satisfying $\lambda_{0}^{3}-\lambda_{0}^{2}-\gamma / 4 \kappa^{2}=0$, where $\gamma$ is the constant in the Diophantine condition (7), and $\kappa$ is the condition number (33).

Proof. From the definitions of $v_{2}$ and $v_{3}$, it is clear that $T v_{2}=\mu_{2} v_{2}-\mu_{3} v_{3}$ and $T v_{3}=\mu_{3} v_{2}+\mu_{2} v_{3}$, and hence $T=C D C^{-1}$, where we define $D=\left(\begin{array}{ccc}\lambda & 0 & 0 \\ 0 & \mu_{2} & \mu_{3} \\ 0 & -\mu_{3} & \mu_{2}\end{array}\right)$. Since $D^{\top} D=\operatorname{diag}\left(\lambda^{2}, \mu_{2}^{2}+\mu_{3}^{2}, \mu_{2}^{2}+\mu_{3}^{2}\right)$, and using the inequalities $\sqrt{\mu_{2}^{2}+\mu_{3}^{2}}=$ $\left|\lambda_{2}\right|<1<\lambda$, one readily sees that $|D|=\lambda$ and we deduce that $\lambda \leq|T| \leq \kappa \lambda$. Now, we use (30), and apply the Diophantine condition (7) to the vector $k=T_{(1)}-(1,0,0)=$ $\left(T_{11}-1, T_{12}, T_{13}\right)$ (it is clear that $k \neq 0$, otherwise $T$ has an integer eigenvalue):

$$
\lambda-1=\langle k, \omega\rangle \geq \frac{\gamma}{|k|^{2}} \geq \frac{\gamma}{4|T|^{2}} \geq \frac{\gamma}{4 \kappa^{2} \lambda^{2}},
$$

where we used that $|k| \leq\left|T_{(1)}\right|+|(1,0,0)| \leq|T|+1 \leq 2|T|$. Finally, a simple study of the function $g(x)=x^{3}-x^{2}-\gamma / 4 \kappa^{2}$ shows that $\lambda>\lambda_{0}$.

Using this lemma, we next show the "uniqueness" of the matrix $T$ satisfying Koch's result. More precisely, we can choose $T=T(\omega) \in \operatorname{SL}(3, \mathbb{Z})$ whose real eigenvalue $\lambda=\lambda(\omega)>1$ is minimal or, equivalently, the norm $|T|$ is minimal. We call this matrix $T$ "the principal Koch's matrix for $\omega$ ".

Proposition 4. There exists a unique matrix $T=T(\omega) \in \operatorname{SL}(3, \mathbb{Z})$ such that all Koch's matrices for $\omega$ have the form $\pm T^{n}, n \geq 1$.

Proof. As we said before, we can restrict ourselves to Koch's matrices of positive determinant. Assume that $T$ and $S$ are two Koch's matrices, with real eigenvalues satisfying $1<\lambda_{T} \leq \lambda_{S}$. It is clear that $S T^{-1}$ has $\omega$ as an eigenvector with eigenvalue $\lambda_{S} / \lambda_{T} \geq 1$, and hence $>1$ (it cannot be equal to 1 ). This says that $S T^{-1}$ is another Koch's matrix, with $\lambda_{S} / \lambda_{T}>\lambda_{0}$ by Lemma 3 (recall that $\lambda_{0}=\lambda_{0}(\omega)>1$ ). Therefore, the real eigenvalues of the Koch's matrices for $\omega$ are all different, and separated at least by a factor $\lambda_{0}$ (filling in this way a discrete set). On the other hand, such eigenvalues satisfy the lower bound given in Lemma 3. This implies that we can choose a Koch's matrix $T=T(\omega)$ with minimal eigenvalue $\lambda=\lambda(\omega)>1$. Then, the matrices $T^{n}$ (and the opposite ones

$\approx$


$\left.-T^{n}\right), n \geq 1$, are also clearly Koch's matrices. It remains to show that they are the only ones. Indeed, if there exists another Koch's matrix $S$, its real eigenvalue satisfies $\lambda^{n}<\lambda_{S}<\lambda^{n+1}$ for some $n \geq 1$, and we deduce that $S T^{-n}$ is a Koch matrix whose eigenvalue satisfies $1<\lambda_{S} \lambda^{-n}<\lambda$, which contradicts our choice of $T$.

Now, our aim is to describe a simple procedure allowing us to determine the principal Koch's matrix for a given cubic vector $\omega$. The idea of our method is that any matrix $T$ with integer (or rational) entries having $\omega$ as an eigenvector is determined by its first row $T_{(1)}=\left(T_{11}, T_{12}, T_{13}\right)$. The matrices $T$ obtained in this way belong to the general linear group $\mathrm{GL}(3, \mathbb{Q})$ but, in general, do not belong to $\operatorname{SL}(3, \mathbb{Z})$. However, we can can explore such matrices by giving successive values to the entries of $T_{(1)}$, until we find a Koch's matrix. First, in the next lemma we establish the (linear) dependence of $T$ with respect to its first row.

Lemma 5. For any vector $T_{(1)}=\left(T_{11}, T_{12}, T_{13}\right)$ with rational entries, there exists a unique matrix $T$ with rational entries, having $\omega$ as an eigenvector, and $T_{(1)}$ as the first row. This matrix can be written as

$$
T=A\left(T_{11} \mathrm{Id}+T_{12} R+T_{13}\left(a_{0} \mathrm{Id}+a_{1} R+a_{2} R^{2}\right)\right) A^{-1},
$$

where we define

$$
R:=\left(\begin{array}{ccc}
0 & 1 & 0 \\
0 & 0 & 1 \\
r_{0} & r_{1} & r_{2}
\end{array}\right)
$$

(recall the coefficients $r_{j}, a_{j}$ and the matrix A, introduced in (22-23) and (27)).

Proof. We begin by proving the result for the particular case of a frequency vector $\omega^{(0)}$ as in (26). It is straightforward to check that the matrix $R$ has $\omega^{(0)}$ as an eigenvector with eigenvalue $\Omega$. The matrix $R^{2}$, which has $(0,0,1)$ has the first row, also has the same eigenvector $\omega^{(0)}$ with eigenvalue $\Omega^{2}$. Then, it is clear that, for any given vector $T_{(1)}^{(0)}=\left(T_{11}^{(0)}, T_{12}^{(0)}, T_{13}^{(0)}\right)$, the matrix

$$
T^{(0)}=T_{11}^{(0)} \mathrm{Id}+T_{12}^{(0)} R+T_{13}^{(0)} R^{2}
$$

has $T_{(1)}^{(0)}$ as the first row, and $\omega^{(0)}$ as an eigenvector with eigenvalue

$$
\lambda=\left\langle T_{(1)}^{(0)}, \omega^{(0)}\right\rangle=T_{11}^{(0)}+T_{12}^{(0)} \Omega+T_{13}^{(0)} \Omega^{2} .
$$

To show the uniqueness of such a matrix, notice that its second and third rows $T_{(2)}^{(0)}$ and $T_{(3)}^{(0)}$ can be determined by the first one using the equalities $\lambda \Omega=\left\langle T_{(2)}^{(0)}, \omega^{(0)}\right\rangle$ and $\lambda \Omega^{2}=\left\langle T_{(3)}^{(0)}, \omega^{(0)}\right\rangle$. which allow us to determine their entries as (rational) coefficients in the basis $1, \Omega, \Omega^{2}$ of the field $\mathbb{Q}(\Omega)$. This shows the result for the particular case of a vector $\omega^{(0)}$.

Now, we consider the general case of a frequency vector $\omega=A \omega^{(0)}$, with a matrix $A$ as in (27). If a matrix $T$ has $\omega$ as an eigenvector and $T_{(1)}=\left(T_{11}, T_{12}, T_{13}\right)$ as the first

\begin{tabular}{|l|l|l|l|l|l|}
\hline 220 & $\mathbf{3 8 3 2}$ & $\mathbf{B}$ & $\begin{array}{l}\text { Dispatch: 13/8/2020 } \\
\text { Total pages: 46 } \\
\text { Disk Received } \\
\text { Disk Used } \square\end{array}$ & $\begin{array}{l}\text { Journal: Commun. Math. Phys. } \\
\text { Not Used } \square \\
\text { Corrupted } \square \\
\text { Mismatch } \square\end{array}$ \\
\hline Jour. No & Ms. No.
\end{tabular}


row, then it has the form $T=A T^{(0)} A^{-1}$, where $T^{(0)}$ has $\omega^{(0)}$ as an eigenvector, with the same eigenvalue

$$
\left\langle T_{(1)}^{(0)}, \omega^{(0)}\right\rangle=\lambda=\left\langle T_{(1)}, \omega\right\rangle=\left\langle A^{\top} T_{(1)}, \omega^{(0)}\right\rangle
$$

(recall that we consider the rows as column vectors). Using again that the entries of the vectors can be determined as coefficients in the basis $1, \Omega, \Omega^{2}$, we deduce that

$$
T_{(1)}^{(0)}=A^{\top} T_{(1)}=\left(T_{11}+a_{0} T_{13}, T_{12}+a_{1} T_{13}, a_{2} T_{13}\right) .
$$

Applying (36), we get the whole matrix $T^{(0)}$ and, performing the linear change given by $A$, we get $T$ as in (34). Its uniqueness is a direct consequence of the uniqueness of $T^{(0)}$.

Now, in order to determine the principal Koch's matrix for $\omega$ we can carry out the following simple exploration. We consider the (integer) entries of the first row $T_{(1)}$ as successive data, say with increasing norm $\left|T_{(1)}\right|$, until the whole matrix $T$ determined from Lemma 5 belongs to $\operatorname{SL}(3, \mathbb{Z})$ (i.e. it has integer entries and determinant 1$)$ and has an eigenvalue $\lambda>1$ in (30). By Koch's result, we know that such a matrix exists and will be reached after a finite exploration. It remains to check whether the matrix $T^{*}$ obtained in this way is the principal Koch's matrix for $\omega$ since, in principle, there could exist another Koch's matrix $T$ with $\left|T_{(1)}\right| \geq\left|T_{(1)}^{*}\right|$ but $|T|<\left|T^{*}\right|$. If this happens, such a new matrix $T$ would satisfy $\left|T_{(1)}\right|<\left|T^{*}\right|$. Hence, after obtaining a first matrix $T^{*}$, it is enough to continue the exploration with increasing norms $\left|T_{(1)}\right|$ up to the value $\left|T^{*}\right|$ and, if a new Koch's matrix $T$ is obtained, check if its norm $|T|$ is lower than $\left|T^{*}\right|$, which would imply that the matrix $T$ has to replace $T^{*}$ as the principal one.

Remark 6. In some particular cases, we can provide directly the matrix $A R A^{-1}$ or its inverse $A R^{-1} A^{-1}$ as a Koch matrix. This will happen if the coefficients $r_{j}$ and $a_{j}$ introduced in (22-23) are all integer, and $\left|r_{0}\right|=\left|a_{2}\right|=1$. Since det $R=r_{0}$ and $\operatorname{det} A=a_{2}$, both of the matrices given above are unimodular (with integer entries and determinant \pm 1 ). Moreover, they have $\omega$ as eigenvector, with eigenvalue $\Omega$ or $\Omega^{-1}$, respectively. Notice also that $\Omega$ and $r_{0}$ have the same sign (indeed, this comes from the fact that the other two eigenvalues $\Omega_{2}, \bar{\Omega}_{2}$ of $R$ are complex, and $r_{0}=\Omega \cdot \Omega_{2} \cdot \bar{\Omega}_{2}$ ). We deduce:

- if $|\Omega|>1$, the matrix $T=r_{0} A R A^{-1}$ is a Koch's matrix, with the eigenvalue $\lambda=r_{0} \Omega>1$;

- if $|\Omega|<1$, the matrix $T=r_{0} A R^{-1} A^{-1}=-A\left(r_{1} \mathrm{Id}+r_{2} R-R^{2}\right) A^{-1}$ is a Koch's matrix, with the eigenvalue $\lambda=r_{0} \Omega^{-1}>1$.

However, the Koch's matrix obtained in this way might not be the principal one, and hence the exploration described above, using the matrices $T$ given by Lemma 5, would be necessary also in this case.

See also in Sect. 2.3 the concrete application of the procedure described above (including Remark 6) to the case of the cubic golden vector. We also recall here that a more systematic algorithm was developed in [DGG16] for the case of a quadratic 2dimensional vector $\omega=(1, \Omega)$, providing a $(2 \times 2)$-matrix $T$, from the (eventually periodic) continued fraction of the frequency ratio $\Omega$.

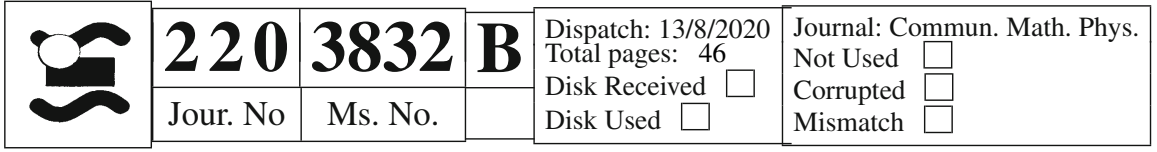


Thus, in view of Proposition 4, we will always assume that $T=T(\omega)$ is the principal Koch's matrix. Since det $T=1$, it is clear that the modulus the two conjugate eigenvalues is $\left|\lambda_{2}\right|=\left|\bar{\lambda}_{2}\right|=\lambda^{-1 / 2}$. We now define the following important number,

$$
\phi=\phi(\omega):=\frac{1}{\pi} \arg \left(\lambda_{2}\right), \quad \text { i.e. } \quad \lambda_{2}, \bar{\lambda}_{2}=\frac{1}{\sqrt{\lambda}} \mathrm{e}^{ \pm \mathrm{i} \pi \cdot \phi},
$$

and we can assume that it is positive: $0<\phi<1$. Indeed, once the matrix $T(\omega)$ is chosen as the principal one, the sign of $\phi$ (or equivalently the sign on $\mu_{3}$ in (31)) is determined by the suitable choice of the sign $s$ for $\sigma_{3}$ in (25).

The next lemma has a crucial role in showing that the function $h_{1}(\varepsilon)$, appearing in the exponent of the maximal splitting distance in Theorem 1, is quasiperiodic, and not periodic, with respect to $\ln \varepsilon$. This comes from the fact that the ratio between the two frequencies of $h_{1}(\varepsilon)$ is given by $\phi$, as we show in Sect. 3.2.

\section{Lemma 7. The number $\phi=\phi(\omega)$ is irrational.}

Proof. Let us assume that $\phi$ is rational, say $\phi=m / n$ as an irreducible fraction. Then, the matrix $T^{n}$ also satisfies Koch's result, but it has $\lambda^{n}$ as a simple eigenvalue, and $(-1)^{m} \lambda^{-n / 2}$ as a double real eigenvalue, which contradicts two facts: the eigenvalues of $T^{n}$ are all simple, and two of them are complex.

\subsection{Quasi-resonances of a cubic frequency vector. The matrix $T$ given by Koch's result} [Koc99] provides approximations to the direction of $\omega=(1, \Omega, \widetilde{\Omega})$. However, we are not interested in finding approximations to $\omega$ but, on the contrary, approximations to the quasi-resonances of $\omega$, which lie close to the "resonant plane" $\langle\omega\rangle^{\perp}$ (the orthogonal plane to $\omega$ ). To be more precise, we say that an integer vector $k \in \mathbb{Z}^{3} \backslash\{0\}$ is a quasi-resonance of $\omega$ if

$$
|\langle k, \omega\rangle|<\frac{1}{2},
$$

and we denote by $\mathcal{A}$ the set of quasi-resonances.

For any given number $x \in \mathbb{R}$, we denote $\operatorname{rint}(x)$ and $\|x\|$ the closest integer to $x$ and the distance from $x$ to such closest integer, respectively. It is clear that $\|x\|=$ $|x-\operatorname{rint}(x)|=\min _{p \in \mathbb{Z}}|x-p|$. Since the first component of $\omega$ is equal to 1 , for any quasiresonance $k=\left(k_{1}, k_{2}, k_{3}\right) \in \mathcal{A}$ we have $\operatorname{rint}\left(k_{2} \Omega+k_{3} \widetilde{\Omega}\right)=-k_{1}$. In other words, for any $q \in \mathbb{Z}^{2} \backslash\{0\}$ we have a quasi-resonance

$$
k^{0}(q):=(-p, q)=\left(-p, q_{1}, q_{2}\right), \text { with } p=p^{0}(q):=\operatorname{rint}\left(q_{1} \Omega+q_{2} \widetilde{\Omega}\right),
$$

whose small divisor is

$$
r_{q}:=\left\langle k^{0}(q), \omega\right\rangle=-p+q_{1} \Omega+q_{2} \widetilde{\Omega}=\left\|q_{1} \Omega+q_{2} \widetilde{\Omega}\right\| .
$$

We also say that $k^{0}(q)$ is an essential quasi-resonance if it is not a multiple of another integer vector, and we denote by $\mathcal{A}_{0}$ the set of essential quasi-resonances.

Now, we define the matrix

$$
U:=\left(T^{-1}\right)^{\top},
$$

\begin{tabular}{|l|l|l|l|l|l|}
\hline 220 & $\mathbf{3 8 3 2}$ & $\mathbf{B}$ & $\begin{array}{l}\text { Dispatch: 13/8/2020 } \\
\text { Total pages: 46 } \\
\text { Disk Received } \\
\text { Disk Used } \square\end{array}$ & $\begin{array}{l}\text { Journal: Commun. Math. Phys. } \\
\text { Not Used } \square \\
\text { Corrupted } \square \\
\text { Mismatch } \square\end{array}$ \\
\hline Jour. No & Ms. No.
\end{tabular}


which satisfies the following simple but important equality:

$$
\langle U k, \omega\rangle=\left\langle k, U^{\top} \omega\right\rangle=\frac{1}{\lambda}\langle k, \omega\rangle
$$

where $\lambda=\lambda(\omega)$ is the eigenvalue of $T$ with $\lambda>1$. This says that successive iterations $U^{n} k$ from a given integer vector $k$ get closer and closer to the resonant plane $\langle\omega\rangle^{\perp}$.

We deduce from (42) that if $k \in \mathcal{A}$, then also $U k \in \mathcal{A}$. We say that the vector $k$ is primitive if $k \in \mathcal{A}$ but $U^{-1} k \notin \mathcal{A}$. It is clear that $k$ is primitive if and only if the following fundamental property is fulfilled:

$$
\frac{1}{2 \lambda}<|\langle k, \omega\rangle|<\frac{1}{2}
$$

Writing $k=k^{0}(q)=(-p, q)$, we denote by $\mathcal{P}$ the set of vectors $q=\left(q_{1}, q_{2}\right) \in \mathbb{Z}^{2} \backslash\{0\}$ associated to primitive vectors:

$$
\mathcal{P}:=\left\{q \in \mathbb{Z}^{2}:\left(q_{1} \geq 1 \text { or }\left(q_{1}=0, q_{2} \geq 1\right)\right) \text { and } k^{0}(q) \text { is primitive }\right\},
$$

where the choice of $q_{1}$ being positive allows us to avoid repetitions, since it means that $k^{0}(q) \in \mathcal{Z}$ (recall the definition (10)). We also denote by $\mathcal{P}_{0}$ the set of vectors $q \in \mathcal{P}$ such that $k^{0}(q)$ is essential.

Now we define, for each $q \in \mathcal{P}$, a resonant sequence of integer vectors:

$$
s(q, n):=U^{n} k^{0}(q), \quad n \geq 0 .
$$

By construction, the set of such resonant sequences covers the whole set of quasiresonances $\mathcal{A}$, providing a classification for them. As done in [DG03,DGG16] for the case of quadratic frequencies, we are going to establish the properties of the resonant sequences (45) for cubic frequencies (see Proposition 11 below).

Remark 8. A resonant sequence $s(q, n)$ generated by an essential primitive $k^{0}(q)$ cannot be a multiple of another resonant sequence. Indeed, in this case we would have $k^{0}(q)=$ $\operatorname{cs}\left(\tilde{q}, n_{0}\right)$ with $|c|>1$ and $n_{0} \geq 0$, and hence $k^{0}(q)$ would not be essential.

Analogously to the basis of eigenvectors $\omega, v_{2} \pm \mathrm{i} v_{3}$ of $T$ introduced in (32), we also consider a basis of eigenvectors of $U$ writing the complex ones in terms of real and imaginary parts:

$$
u_{1}, \quad u_{2}+\mathrm{i} u_{3}=\sigma\left(u_{1}\right), \quad u_{2}-\mathrm{i} u_{3}=\bar{\sigma}\left(u_{1}\right),
$$

with eigenvalues $\lambda^{-1}, \lambda_{2}^{-1}, \bar{\lambda}_{2}^{-1}$, respectively. One readily sees that $\left\langle u_{2}, \omega\right\rangle=\left\langle u_{3}, \omega\right\rangle=$ 0 , i.e. $u_{2}$ and $u_{3}$ span the resonant plane $\langle\omega\rangle^{\perp}$. Other useful equalities are: $\left\langle u_{1}, v_{2}\right\rangle=$ $\left\langle u_{1}, v_{3}\right\rangle=0,\left\langle u_{2}, v_{2}\right\rangle=-\left\langle u_{3}, v_{3}\right\rangle,\left\langle u_{2}, v_{3}\right\rangle=\left\langle u_{3}, v_{2}\right\rangle$. We define $Z_{1}, Z_{2}$ and $\theta$ through the formulas

$$
\frac{1}{2}\left(\left|u_{2}\right|^{2}+\left|u_{3}\right|^{2}\right)=Z_{1}, \quad \frac{1}{2}\left(\left|u_{2}\right|^{2}-\left|u_{3}\right|^{2}\right)=Z_{2} \cos \theta, \quad\left\langle u_{2}, u_{3}\right\rangle=Z_{2} \sin \theta,
$$

and the following important number,

$$
\delta=\delta(\omega):=\frac{Z_{2}}{Z_{1}}
$$

\begin{tabular}{|l|l|l|l|l|l|}
\hline $\mathbf{2} 2 \mathbf{2 0}$ & $\mathbf{3 8 3 2}$ & $\mathbf{B}$ & $\begin{array}{l}\text { Dispatch: 13/8/2020 } \\
\text { Total pages: 46 } \\
\text { Disk Received } \\
\text { Disk Used } \square\end{array}$ & $\begin{array}{l}\text { Journal: Commun. Math. Phys. } \\
\text { Not Used } \square \\
\text { Corrupted } \\
\text { Mismatch } \square\end{array}$ \\
\hline Jour. No & Ms. No.
\end{tabular}


It is clear, from the definition of $Z_{1}$ and $Z_{2}$, that $0 \leq \delta \leq 1$. The following result shows that $\delta$ cannot achieve the extreme values 0 and 1 . In particular, the fact that $\delta>0$ has a crucial role (together with the irrationality of $\phi$ shown in Lemma 7) in showing that the quasiperiodic function $h_{1}(\varepsilon)$, appearing in the exponent of the maximal splitting distance in Theorem 1, is not periodic with respect to $\ln \varepsilon$.

Lemma 9. The number $\delta=\delta(\omega)$ satisfies $0<\delta<1$.

Proof. We first show that $\delta<1$. Indeed, if $\delta=1$ then $Z_{1}=Z_{2}$, which would imply that $\left|\left\langle u_{2}, u_{3}\right\rangle\right|=\left|u_{2}\right| \cdot\left|u_{3}\right|$, but this is not possible since $u_{2}$ and $u_{3}$ are linearly independent.

Now, we are going to see that $\delta>0$. If we have $\delta=0$, then $Z_{2}=0$ and, from (47), the expressions $\left|u_{2}\right|^{2}-\left|u_{3}\right|^{2}$ and $\left\langle u_{2}, u_{3}\right\rangle$ would vanish simultaneously. To show that this is not possible, we are going to see that they can be written as follows,

$$
\left|u_{2}\right|^{2}-\left|u_{3}\right|^{2}=c_{0}+c_{1} \Omega+c_{2} \Omega^{2}, \quad\left\langle u_{2}, u_{3}\right\rangle=\left(d_{0}+d_{1} \Omega+d_{2} \Omega^{2}\right) \sigma_{3}
$$

(see (24) for $\sigma_{3}$ ) and that the coefficients $c_{j}, d_{j}$ cannot be all zero.

Let us write the coefficients $c_{j}, d_{j}$ as rational expressions in the coefficients $r_{j}$, $a_{j}$ introduced in (22-23). Recall that, in (46), we introduced $u_{2} \pm \mathrm{i} u_{3}$ as complex eigenvectors of the matrix $U$, conjugates of the real eigenvector $u_{1}$. It is clear from (41) that the eigenvectors of $U$ are the same as for $T^{\top}$. Since the matrix $T$ can be written as in (34) (with suitable coefficients $T_{1 j}$ ), it is easy to relate the eigenvectors of $T^{\top}$ with the ones of $R^{\top}$, through the linear change defined by the matrix $B:=\left(A^{-1}\right)^{\top}$, where $A$ is the matrix introduced in (27). Namely, we have

$$
u_{1}=B u_{1}^{(0)}, \quad u_{2} \pm \mathrm{i} u_{3}=B\left(u_{2}^{(0)} \pm \mathrm{i} u_{3}^{(0)}\right)
$$

where $u_{1}^{(0)}, u_{2}^{(0)} \pm \mathrm{i} u_{3}^{(0)}=\sigma\left(u_{1}^{(0)}\right), \bar{\sigma}\left(u_{1}^{(0)}\right)$ are the eigenvectors of $R^{\top}$. Using (35) and the cubic Eq. (22), it is not hard to obtain the real eigenvector $u_{1}^{(0)}$ (with eigenvalue $\Omega$ ) and, subsequently, the complex eigenvectors $u_{2}^{(0)} \pm \mathrm{i} u_{3}^{(0)}$ as its conjugates (with eigenvalues $\sigma_{2} \pm \mathrm{i} \sigma_{3}$, recall (24)). We get

$$
\begin{aligned}
& u_{1}^{(0)}=\left(r_{0},-r_{2} \Omega+\Omega^{2}, \Omega\right), \\
& u_{2}^{(0)}=\left(r_{0},-r_{2} \sigma_{2}+\sigma_{2}^{2}-\sigma_{3}^{2}, \sigma_{2}\right), \quad u_{3}^{(0)}=\sigma_{3}\left(0,-r_{2}+2 \sigma_{2}, 1\right) .
\end{aligned}
$$

Using such ingredients, together with (25), we are able to obtain algebraic expressions for (49) in the basis $1, \Omega, \Omega^{2}$ of the field $\mathbb{Q}(\Omega)$. After some tedious computations, we get the following coefficients:

$$
\begin{aligned}
c_{0}= & r_{0}^{2}-\left(\frac{a_{0}}{a_{2}}+\frac{1}{2}\right) r_{0} r_{2}-\frac{2 a_{1}}{a_{2}} r_{0}+r_{1}^{2}-\frac{a_{1}}{a_{2}} r_{1} r_{2} \\
& +\frac{a_{0}^{2}+a_{1}^{2}+1}{a_{2}^{2}}\left(r_{1}+\frac{r_{2}^{2}}{2}\right), \\
c_{1}= & \left(\frac{a_{0}}{a_{2}}-\frac{1}{2}\right) r_{0}+\left(\frac{r_{2}}{2}+\frac{a_{1}}{a_{2}}\right) r_{1}, \quad c_{2}=-\frac{r_{1}}{2}-\frac{a_{0}^{2}+a_{1}^{2}+1}{2 a_{2}^{2}}, \\
d_{0}= & -\left(c_{1}+r_{2} c_{2}\right), \quad d_{1}=c_{2}, \quad d_{2}=0 .
\end{aligned}
$$


Assuming $c_{j}=d_{j}=0, j=0,1,2$, we reach a contradiction. Indeed, from $c_{2}=0$ we get $r_{1}=-\left(a_{0}^{2}+a_{1}^{2}+1\right) / a_{2}^{2}$ and, replacing into the remaining coefficients, we obtain

$$
\begin{aligned}
& c_{1}=-d_{0}=\left(\frac{a_{0}}{a_{2}}-\frac{1}{2}\right) r_{0}-\frac{a_{0}^{2}+a_{1}^{2}+1}{a_{2}^{2}}\left(\frac{r_{2}}{2}+\frac{a_{1}}{a_{2}}\right), \\
& c_{0}+c_{1} r_{2}=r_{0}^{2}-\left(r_{2}+\frac{2 a_{1}}{a_{2}}\right) r_{0} .
\end{aligned}
$$

Since $r_{0} \neq 0$ in (22), from the second equality we get $r_{0}=r_{2}+2 a_{1} / a_{2}$ and the first equality becomes

$$
c_{1}=-\left(\left(\frac{a_{0}}{a_{2}}-1\right)^{2}+\frac{a_{1}^{2}+1}{a_{2}^{2}}\right) \frac{r_{0}}{2},
$$

which contradicts our assumption that $c_{1}=0$ and, consequently, we have $\delta>0$.

Remark 10. The previous arguments show, for the numbers defined in (47), that we have $Z_{1}, Z_{2}^{2} \in \mathbb{Q}(\omega)$. Indeed, using the rational expressions obtained for the coefficients $c_{j}$, $d_{j}$ (together with the fact that $\sigma_{3}^{2} \in \mathbb{Q}(\omega)$ ), we can determine from (49) the coefficients of $Z_{2}^{2}$ in the basis $1, \Omega, \Omega^{2}$. In an analogous way, we can determine the coefficients of $Z_{1}$ in the same basis, and we deduce from (48) that $\delta^{2} \in \mathbb{Q}(\Omega)$. Then, it is also possible obtain the coefficients of $\delta^{2}$ in the basis $1, \Omega, \Omega^{2}$ by carrying out a quotient in the field $\mathbb{Q}(\Omega)$, though the general expression is very complicated. See (69) for the particular case of the cubic golden frequency vector.

For any $q \in \mathcal{P}$, we define

$$
y_{q}:=\left\langle k^{0}(q), v_{2}\right\rangle, z_{q}:=\left\langle k^{0}(q), v_{3}\right\rangle,
$$

and $E_{q}, \psi_{q}, K_{q}$ and $\gamma_{q}^{*}$ through the formulas

$$
\frac{\left\langle v_{2}, u_{2}\right\rangle y_{q}+\left\langle v_{2}, u_{3}\right\rangle z_{q}}{\left\langle v_{2}, u_{2}\right\rangle^{2}+\left\langle v_{2}, u_{3}\right\rangle^{2}}=E_{q} \cos \psi_{q}, \quad \frac{\left\langle v_{2}, u_{3}\right\rangle y_{q}-\left\langle v_{2}, u_{2}\right\rangle z_{q}}{\left\langle v_{2}, u_{2}\right\rangle^{2}+\left\langle v_{2}, u_{3}\right\rangle^{2}}=E_{q} \sin \psi_{q},
$$

$$
K_{q}:=E_{q}^{2} Z_{1}, \quad \gamma_{q}^{*}:=\left|r_{q}\right| K_{q} .
$$

We see in the next proposition that any given resonant sequence $s(q, n)$ defined in (45) exhibits an "oscillatory limit behavior" as $n \rightarrow \infty$ : the sizes of the vectors $s(q, n)$ oscillate around a sequence having geometric growth of rate $\lambda^{1 / 2}$, and the numerators $\gamma_{s(q, n)}$ oscillate around the value $\gamma_{q}^{*}$, which can be considered as the "mean Diophantine constant" for the resonant sequence $s(q, n)$. This proposition extends the results given in [DG03,DGG16] for the quadratic case, where a (non-oscillatory) limit behavior is also established for resonant sequences. In our case of a non-totally real complex vector $\omega$, the relative amplitude and the frequency of the oscillations are directly related to the numbers $\phi=\phi(\omega)$ and $\delta=\delta(\omega)$, introduced in (37) and (48) respectively. As we see in Sect. 3, the facts that $\phi$ is irrational and $\delta>0$, shown by Lemmas 7 and 9 respectively, allow us to show that the function $h_{1}(\varepsilon)$ associated to the maximal splitting distance in Theorem 1 , is quasiperiodic but not periodic with respect to $\ln \varepsilon$.

\begin{tabular}{|l|l|l|l|l|l|}
\hline $\mathbf{2} 2 \mathbf{2 0}$ & $\mathbf{3 8 3 2}$ & $\mathbf{B}$ & $\begin{array}{l}\text { Dispatch: 13/8/2020 } \\
\text { Total pages: 46 } \\
\text { Disk Received } \\
\text { Disk Used } \square\end{array}$ & $\begin{array}{l}\text { Journal: Commun. Math. Phys. } \\
\text { Not Used } \square \\
\text { Corrupted } \\
\text { Mismatch } \square\end{array}$ \\
\hline Jour. No & Ms. No.
\end{tabular}


Proposition 11. Let $\omega=(1, \Omega, \widetilde{\Omega})$ be a cubic frequency vector of complex type. Consider $\phi, \theta$ and $\delta$ as defined in (37) and (47-48), and the vector $u_{1}$ as in (46). For any given $q \in \mathcal{P}$, consider $r_{q}, \psi_{q}, K_{q}$ and $\gamma_{q}^{*}$ as defined in (40) and (52-53). Then, the resonant sequence $s(q, \cdot)$ defined in (45) and its associated numerators $\gamma_{s(q, \cdot)}$ satisfy the approximations

$$
\begin{aligned}
|s(q, n)|^{2} & =K_{q} b_{s(q, n)} \cdot \lambda^{n}+\mathcal{O}\left(\lambda^{-n / 2}\right), \\
\gamma_{s(q, n)} & =\gamma_{q}^{*} b_{s(q, n)}+\mathcal{O}\left(\lambda^{-3 n / 2}\right),
\end{aligned}
$$

with an oscillating factor defined by

$$
b_{s(q, n)}:=1+\delta \cos \left(2 \pi \cdot n \phi+2 \psi_{q}-\theta\right),
$$

and hence the numerators $\gamma_{s(q, \cdot)}$ oscillate as $n \rightarrow \infty$ between the values

$$
\gamma_{q}^{-}:=\gamma_{q}^{*}(1-\delta), \quad \gamma_{q}^{+}:=\gamma_{q}^{*}(1+\delta) .
$$

Moreover, we have the lower bound

$$
\gamma_{q}^{-} \geq \frac{1-\delta}{2 \lambda(1+\delta)}\left(|q|-Q_{0}\right)^{2}, \text { provided }|q| \geq Q_{0}:=\frac{\left|u_{1}\right|}{2\left|\left\langle u_{1}, \omega\right\rangle\right|} .
$$

For a proof, see [DGG14a].

Remark 12. We just outline here the main facts leading to the dominant behaviors (5455) described by this proposition, and show why this result is valid only in the case of complex conjugates. On one hand, for any given resonant sequence, the size of the vectors $s(q, n)$ increases like $\lambda^{n / 2}$ as $n \rightarrow \infty$ (with an oscillatory factor), since the (coincident) modulus of the greatest eigenvalues of the iteration matrix $U$ is $\lambda^{1 / 2}$. On the other hand, the small divisors $|\langle s(q, n), \omega\rangle|$ decrease like $\lambda^{-n}$ according to the equality (42). Therefore, the numerators $\gamma_{s(q, n)}=|\langle s(q, n), \omega\rangle| \cdot|s(q, n)|^{2}$ become bounded from above and from below. This fact does not apply to the totally real case, in which the conjugates of a cubic irrational number have different modulus.

As we can see in (55), the existence of limit of the sequences $\gamma_{s}(q, n)$, stated in [DGG16] for the quadratic case, is replaced in our complex cubic case by an oscillatory limit behavior, with a lower limit $\liminf _{n \rightarrow \infty} \gamma_{s(q, n)}=\gamma_{q}^{-}$and an upper limit $\limsup _{n \rightarrow \infty} \gamma_{s(q, n)}=\gamma_{q}^{+}$, introduced in (57). Notice that we could give the exact values of such limits due to the irrationality of the phase $\phi$ appearing in the oscillating factors (56), stated in Lemma 7.

As another relevant fact, we stress that the amplitude of the limit oscillations is proportional to the number $\delta$ introduced in (48). Since $\delta>0$ by Lemma 9, we can ensure that such oscillations do occur.

An important consequence of the lower bound (58) is that the minimal value among the values $\gamma_{q}^{*}$ is reached for some concrete $\widehat{q}$. Indeed, the values $\gamma_{q}^{*}$ are not increasing in general with respect to $|q|$, but the increasing lower bound (58) implies that $\lim _{|q| \rightarrow \infty} \gamma_{q}^{*}=$ $\infty$, and one has to check only a finite number of cases in order to detect a vector $\widehat{q}$ providing the minimal value among $\gamma_{q}^{*}, q \in \mathcal{P}$. We define the primary resonances as the integer vectors belonging to the sequence

$$
s_{0}(n):=s(\widehat{q}, n),
$$

\begin{tabular}{|l|l|l|l|l|l|}
\hline 220 & $\mathbf{3 8 3 2}$ & $\mathbf{B} \mathbf{3} \begin{array}{l}\text { Dispatch: 13/8/2020 } \\
\text { Total pages: 46 } \\
\text { Disk Received } \square \\
\text { Disk Used } \square\end{array}$ & $\begin{array}{l}\text { Journal: Commun. Math. Phys. } \\
\text { Not Used } \square \\
\text { Corrupted } \square \\
\text { Mismatch } \square\end{array}$ \\
\hline
\end{tabular}


and we denote

$$
\gamma^{*}:=\min _{q \in \mathcal{P}} \gamma_{q}^{*}=\gamma(\hat{q})>0
$$

which can be considered as the "minimal mean Diophantine constant". The fact that $\gamma^{*}>0$ implies that any non-totally real cubic frequency vector $\omega$ satisfies the Diophantine condition (28) (with the minimal exponent 2), and we can compute explicitly the "asymptotic Diophantine constant" (17):

$$
\liminf _{|k| \rightarrow \infty} \gamma_{k}=\liminf _{n \rightarrow \infty} \gamma_{s_{0}(n)}=\gamma^{*}(1-\delta)=\gamma^{-}>0 .
$$

Dividing by $\gamma^{*}$, we also introduce normalized numerators and their associated asymptotic values, to be used in Sect. 3:

$$
\tilde{\gamma}_{k}:=\frac{\gamma_{k}}{\gamma^{*}}, \quad \tilde{\gamma}_{q}^{*}:=\frac{\gamma_{q}^{*}}{\gamma^{*}}, \quad \tilde{\gamma}_{q}^{ \pm}:=\frac{\gamma_{q}^{ \pm}}{\gamma^{*}},
$$

and in this way we get $\tilde{\gamma}_{\hat{q}}^{*}=1$ for the primary resonances.

Remark 13. (a) In principle, for some particular cubic frequency vectors $\omega$, the minimum in (60) could be reached by two or more vectors $q$ and, consequently, there could exist two or more sequences of primary resonances. In such a case, we denote by $\widehat{q}$ only one of such vectors $q$.

(b) Any primitive vector generating a sequence of primary resonances is essential: $\widehat{q} \in$ $\mathcal{P}_{0}$. Indeed, if $\widehat{q}$ is not essential, then we have $k^{0}(\widehat{q})=\operatorname{cs}\left(\bar{q}, n_{0}\right)$ with $|c|>1$ and $n_{0} \geq 0$, and therefore $s(\widehat{q}, n)=c s\left(\bar{q}, n_{0}+n\right)$, which implies by (29) that $\gamma_{\widehat{q}}^{*}=|c|^{3} \gamma_{\bar{q}}^{*}$, and the minimum in (60) would not be reached for $\widehat{q}$.

We call secondary resonances the vectors belonging to any of the remaining sequences $s(q, n), q \in \mathcal{P} \backslash\{\widehat{q}\}$. We also consider the second minimum in (60):

$$
\min _{q \in \mathcal{P} \backslash\{\widehat{q}\}} \gamma_{q}^{*}=\gamma_{\widehat{q}^{\prime}}^{*}
$$

and we can call "main secondary resonances" the integer vectors in the sequence $s\left(\widehat{q}^{\prime}, n\right)$. It is clear that its associated normalized numerator satisfies $\tilde{\gamma}_{\hat{q}^{\prime}}^{*} \geq 1$.

In order to measure the "separation" between the primary and the secondary resonances, we define the values

$$
\begin{aligned}
& J_{0}^{+}=J_{0}^{+}(\omega):=\left(\tilde{\gamma}_{\widehat{q}}^{+}\right)^{1 / 3}=(1+\delta)^{1 / 3}, \\
& B_{0}^{-}=B_{0}^{-}(\omega):=\left(\tilde{\gamma}_{\widehat{q}^{\prime}}^{-}\right)^{1 / 3}=\left(\tilde{\gamma}_{\widehat{q}^{\prime}}^{*}\right)^{1 / 3}(1-\delta)^{1 / 3}
\end{aligned}
$$

(we included the exponent $1 / 3$ for convenience, see Sect. 3 ). To have a clear distinction between primary and secondary resonances we need the following "weak separation condition":

$$
B_{0}^{-}>J_{0}^{+},
$$

which says the interval $\left[\gamma_{\widehat{q}}^{-}, \gamma_{\hat{q}}^{+}\right]$has no intersection with any other interval $\left[\gamma_{q}^{-}, \gamma_{q}^{+}\right]$, $q \neq \widehat{q}$ (as happens for the cubic golden vector, see the next section).

\begin{tabular}{|l|l|l|l|l|l|}
\hline 220 & $\mathbf{3 8 3 2}$ & $\mathbf{B}$ & $\begin{array}{l}\text { Dispatch: 13/8/2020 } \\
\text { Total pages: 46 } \\
\text { Disk Received } \square \\
\text { Disk Used } \square\end{array}$ & $\begin{array}{l}\text { Journal: Commun. Math. Phys. } \\
\text { Not Used } \square \\
\text { Corrupted } \square \\
\text { Mismatch } \square\end{array}$ \\
\hline
\end{tabular}




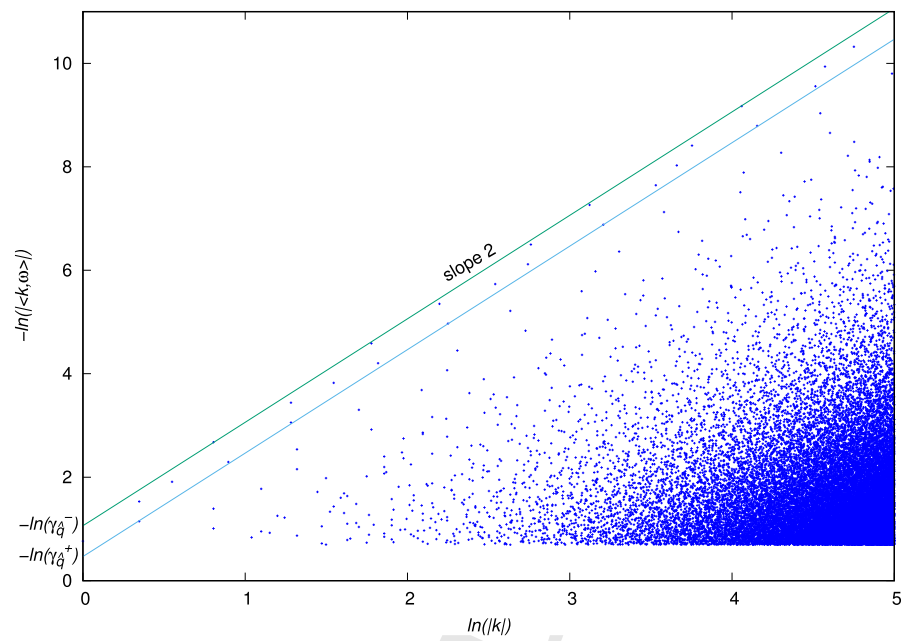

Fig. 2. Points $(x, y)=(\ln |k|,-\ln |\langle k, \omega\rangle|)$ for the cubic golden frequency vector; the primary resonances correspond to the points lying between the two straight lines $y=2 x-\ln \gamma \frac{\mathrm{q}}{ \pm}$

Additionally, it is interesting to visualize the separation between primary and secondary resonances in the following way. Taking logarithm of both sides of the Diophantine condition (7), we can write it as

$$
-\ln |\langle k, \omega\rangle| \leq 2 \ln |k|-\ln \gamma
$$

In Fig. 2 (which corresponds to the cubic golden vector), where we draw all the points with coordinates $(x, y)=(\ln |k|,-\ln |\langle k, \omega\rangle|)$ (up to a large value of $|k|)$, we can see a sequence of points lying between the two straight lines $y=2 x-\ln \gamma \widehat{\underline{q}}$. Those points correspond to integer vectors belonging to the sequence of primary resonances: $k=s_{0}(n), n \geq 0$, and the remaining points correspond to secondary resonances.

2.3. The cubic golden frequency vector. In this section, we provide particular data for the concrete case of the cubic golden frequency vector. We point out that a similar approach could be carried out for other cubic vectors (see [Cha02] for some famous examples).

We introduce $\Omega$ as the real number satisfying $\Omega^{3}=1-\Omega$, which has been called the cubic golden number (see for instance [HK00]). Then, we consider the frequency vector

$$
\omega=\left(1, \Omega, \Omega^{2}\right) \approx(1,0.682328,0.465571)
$$

In other words, the coefficients introduced in (22-23) are $r_{0}=1, r_{1}=-1, r_{2}=0, a_{0}=$ $a_{1}=0, a_{2}=1$, and hence the matrices defined in (35) and (27) are $R=\left(\begin{array}{ccc}0 & 1 & 0 \\ 0 & 0 & 1 \\ 1 & -1 & 0\end{array}\right)$ and $A=$ Id.

\begin{tabular}{|l|l|l|l|l|l|}
\hline 220 & $\mathbf{3 8 3 2}$ & $\mathbf{B}$ & $\begin{array}{l}\text { Dispatch: 13/8/2020 } \\
\text { Total pages: 46 } \\
\text { Disk Received } \\
\text { Disk Used } \square\end{array}$ & $\begin{array}{l}\text { Journal: Commun. Math. Phys. } \\
\text { Not Used } \square \\
\text { Corrupted } \square \\
\text { Mismatch } \square\end{array}$ \\
\hline Jour. No & Ms. No.
\end{tabular}


In fact, we can provide exact expressions for $\Omega$ using some of the standard formulas for the solutions of the general cubic equation (see for instance [Wei03]). We have

$$
\Omega=S_{+}+S_{-}=S_{ \pm}-\frac{1}{3 S_{ \pm}}, \quad \text { with } S_{ \pm}=\sqrt[3]{\frac{1}{2}\left(1 \pm \sqrt{\frac{31}{27}}\right)},
$$

or also

$$
\Omega=\frac{2}{\sqrt{3}} \sinh \left(\frac{1}{3} \operatorname{arsinh} \frac{3 \sqrt{3}}{2}\right) .
$$

It is easy, from the results of Sect. 2.1, to obtain the principal Koch's matrix for the frequency vector (67). By Lemma 5, any Koch's matrix is determined from its first row $T_{(1)}=\left(T_{11}, T_{12}, T_{13}\right)$, by the formula $T=T_{11} \mathrm{Id}+T_{12} R+T_{13} R^{2}$. On the other hand, by Remark 6 we can ensure that $T^{*}=R^{-1}=\mathrm{Id}+R^{2}$ is a Koch's matrix but, in principle, it might not be the principal one. To check whether another Koch's matrix can be the principal one, we carry out the exploration described after Lemma 5 in the following way. We use that the matrix $T^{*}$ given above has norm $\left|T^{*}\right|=(\sqrt{5}+1) / 2 \approx 1.618034$, and its first row $T_{(1)}^{*}=(1,0,1)$ has norm $\left|T_{(1)}^{*}\right|=\sqrt{2} \approx 1.414214$. Then, by exploring the matrices $T$ given by a few possible first rows $T_{(1)}$ (with norms between $\sqrt{2}$ and $(\sqrt{5}+1) / 2)$, we ensure that the Koch's matrix $T^{*}$ given above is the principal one. We rename it as $T$.

In this way, the principal Koch's matrix for the cubic golden frequency vector (67), and the subsequent matrix introduced in (41), are

$$
T=R^{-1}=\left(\begin{array}{lll}
1 & 0 & 1 \\
1 & 0 & 0 \\
0 & 1 & 0
\end{array}\right), \quad U=R^{\top}=\left(\begin{array}{ccc}
0 & 0 & 1 \\
1 & 0 & -1 \\
0 & 1 & 0
\end{array}\right),
$$

with the eigenvalue

$$
\lambda=1+\Omega^{2}=\frac{1}{\Omega} \approx 1.465571
$$

which satisfies $\lambda^{3}=1+\lambda^{2}$.

Let us compute several relevant parameters, defined in Sect. 2.1. Writing the conjugates of $\Omega$ as $\Omega_{2}, \bar{\Omega}_{2}=\sigma_{2} \pm \mathrm{i} \sigma_{3}$, by (25) we have

$$
\sigma_{2}=-\frac{\Omega}{2}, \quad \sigma_{3}=-\frac{\sqrt{4+3 \Omega^{2}}}{2},
$$

where the sign $s=-1$ chosen for $\sigma_{3}$ in (25) ensures that $\lambda_{2}=1 / \Omega_{2}=1 /\left(\sigma_{2}+\mathrm{i} \sigma_{3}\right)$ has positive imaginary part, and hence the the number defined in (37) is

$$
\phi=1+\frac{1}{\pi} \arctan \frac{-\sigma_{3}}{\sigma_{2}} \approx 0.590935,
$$

and it is irrational by Lemma 7. As stated in Theorem 1, the number $\phi$ is the frequency ratio of the function $h_{1}(\varepsilon)$ as a quasiperiodic funtion (with respect to $\ln \varepsilon$ ). It is interesting to consider its (infinite) continued fraction and its associated convergents, whose

二


denominators provide "approximate periods" for $h_{1}(\varepsilon)=F_{1}(\zeta)$ (in the logarithmic variable $\zeta \sim \ln (1 / \varepsilon)$, see $(81))$ :

$$
\phi=[0 ; 1,1,2,4,78, \ldots] \approx \frac{1}{1}, \frac{1}{2}, \frac{3}{5}, \frac{13}{22}, \frac{1017}{1721}, \ldots
$$

In particular, the convergent $13 / 22$ is close enough to $\phi$, and explains the fact that $F_{1}(\zeta)$ appears to be 22-periodic in Fig. 1. On the other hand, the number $\delta$ introduced in (48) can be obtained by carrying out, for this particular case, the computations described in Remark 10, and we get

$$
\delta=\sqrt{-1+5 \Omega-5 \Omega^{2}} \approx 0.289453
$$

In the table below, we write down several numerical data appearing in Proposition 11, for the resonant sequences $s(q, n)$ induced by the primitives $k^{0}(q)$ (see (39) and (45)): the numbers $\gamma_{q}^{*}$, the bounds $\gamma_{q}^{-}$and $\gamma_{q}^{+}$, and the normalized values $\tilde{\gamma}_{q}^{*}$ (defined in (5153), (57) and (62), respectively; we also use the expressions (32) and (50) for the vectors $v_{j}$ and $\left.u_{j}\right)$. We restrict such data to the primitives $k^{0}(q)$ with $|q|<3$, and we provide a lower bound for all other primitives (see (58)).

\begin{tabular}{lllll}
\hline$k^{0}(q)=(-p, q)$ & $\gamma_{q}^{-}$ & $\gamma_{q}^{*}$ & $\gamma_{q}^{+}$ & $\tilde{\gamma}_{q}^{*}$ \\
\hline$(0,0,1)$ & 0.345858 & 0.486749 & 0.627640 & 1 \\
$(-1,2,0)$ & 1.037575 & 1.460248 & 1.882920 & 3 \\
$(-2,1,2)$ & 3.112725 & 4.380743 & 5.648761 & 9 \\
$(0,2,-2)$ & 2.766867 & 3.893994 & 5.021121 & 8 \\
$|q| \geq 3$ & $\geq 1.274218$ & & & \\
\hline
\end{tabular}

As we see from this table, the smallest value of $\gamma_{q}^{*}$ corresponds to $\widehat{q}=(0,1)$, i.e. to the primitive vector $k^{0}(\widehat{q})=(0,0,1)$, which generates the sequence of primary resonances. The minimum of the values $\gamma_{q}^{*}$ is the "minimal mean Diophantine constant" introduced in (60):

$$
\gamma^{*}=\gamma_{\widehat{q}}^{*}=\frac{2}{31}\left(5+\Omega+4 \Omega^{2}\right) \approx 0.486749
$$

(the algebraic expression in the basis $1, \Omega, \Omega^{2}$ has also been obtained from the definition (51-53), working in the field $\mathbb{Q}(\Omega)$ ). On the other hand, we get for the "asymptotic Diophantine constant" $(61)$ the value $\gamma^{-} \approx 0.345858$. Other numerical values appearing in Proposition 11 are $\theta \approx-1.054837$ and $\psi_{\widehat{q}} \approx-2.007416$ (the latter one for the primary resonances), defined in (47) and (52) respectively.

Finally, in (64-65) we get

$$
J_{0}^{+}=(1+\delta)^{1 / 3} \approx 1.088433, \quad B_{0}^{-}=3^{1 / 3}(1-\delta)^{1 / 3} \approx 1.286979,
$$

and hence the weak separation condition (66) is fulfilled.

\section{Searching for the Asymptotic Estimate}

In order to provide an asymptotic estimate for the splitting, given in our main result (Theorem 1) in terms of the splitting function $\mathcal{M}(\theta)$, we first need to carry out a careful study of the first order approximation (3) provided by the Poincaré-Melnikov method. Although this approximation is given by the (vector) Melnikov function $M(\theta), \theta \in \mathbb{T}^{3}$, 
it is more convenient to work with the (scalar) splitting potential $L(\theta)$, whose gradient is the Melnikov function: $\nabla L(\theta)=M(\theta)$.

In this section, we provide the constructive part of the proof, which amounts to find, for every sufficiently small $\varepsilon$, the dominant harmonic of the Fourier expansion of the Melnikov potential $L(\theta)$, with an asymptotic estimate for its size of the type $\exp \left\{-h_{1}(\varepsilon) / \varepsilon^{1 / 6}\right\}$, with an oscillating (positive) function $h_{1}(\varepsilon)$ in the exponent. This function can be explicitly defined from the arithmetic properties of our cubic frequency vector $\omega$ and, as a direct consequence, we see that it is quasiperiodic (and continuous) with respect to $\ln \varepsilon$, and hence bounded (and we provide concrete lower and upper bounds for it). We can also study, from such arithmetic properties, whether the dominant harmonic is always given by a primary resonance (providing a sufficient condition for this, which is satisfied in the case of the cubic golden frequency vector) or, otherwise, secondary resonances can be dominant for some intervals of $\varepsilon$.

The final step, considered in Sect. 4, requires to ensure that the whole Melnikov function $M(\theta)$ is dominated by its dominant harmonic, by obtaining a bound for the sum of all the remaining harmonics of its Fourier expansion. Furthermore, to ensure that the Poincaré-Melnikov method (3) predicts correctly the size of the splitting in the singular case $\mu=\varepsilon^{r}$, one has to extend the results to the splitting function $\mathcal{M}(\theta)$ by showing that the asymptotic estimate of the dominant harmonic is large enough to overcome the harmonics of the error term in (3). This step is just outlined in Sect. 4, since it is analogous to the one already done in [DG04] for the case of the quadratic golden number (using the upper bounds for the error term provided in [DGS04]).

3.1. Estimates of the harmonics of the splitting potential. We plug our functions $f$ and $h$, defined in (9), into the integral (16) and get the Fourier expansion of the Melnikov potential, where the coefficients can be obtained using residues (see for instance [DG00, Sect. 3.3]):

$$
L(\theta)=\sum_{k \in \mathcal{Z} \backslash\{0\}} L_{k} \cos \left(\langle k, \theta\rangle-\sigma_{k}\right), \quad L_{k}=\frac{2 \pi\left|\left\langle k, \omega_{\varepsilon}\right\rangle\right| \mathrm{e}^{-\rho|k|}}{\sinh \left|\frac{\pi}{2}\left\langle k, \omega_{\varepsilon}\right\rangle\right|},
$$

where it is clear that $L_{k}>0$, and the phases $\sigma_{k}$ are the same as in (9). Recalling that the fast frequencies $\omega_{\varepsilon}$ are given in (1) and taking into account the definition of the numerators $\gamma_{k}$ in (29), we can present each coefficient $L_{k}=L_{k}(\varepsilon), k \in \mathcal{Z} \backslash\{0\}$ (recall that we introduced the set $\mathcal{Z} \subset \mathbb{Z}^{3}$ in (10), to avoid repetitions in Fourier expansions), in the form

$$
\begin{aligned}
L_{k} & =\alpha_{k} \mathrm{e}^{-\beta_{k}}, \quad \alpha_{k}(\varepsilon) \approx 4 \pi\left|\left\langle k, \omega_{\varepsilon}\right\rangle\right|=\frac{4 \pi \gamma_{k}}{|k|^{2} \sqrt{\varepsilon}}, \\
\beta_{k}(\varepsilon) & =\rho|k|+\frac{\pi}{2}\left|\left\langle k, \omega_{\varepsilon}\right\rangle\right|=\rho|k|+\frac{\pi \gamma_{k}}{2|k|^{2} \sqrt{\varepsilon}},
\end{aligned}
$$

where an exponentially small term has been neglected in the denominator of $\alpha_{k}$. The most relevant term in this expression is $\beta_{k}$, which gives the exponential smallness in $\varepsilon$ of each coefficient, and we will show that $\alpha_{k}$ provides a polynomial factor. For any given $\varepsilon$, the smallest exponents $\beta_{k}(\varepsilon)$ provide the largest (exponentially small) coefficients $L_{k}(\varepsilon)$ and hence the dominant harmonics. Our aim is to study the dependence on $\varepsilon$ of the size of the most dominant harmonic.

\begin{tabular}{|l|l|l|l|l|l|}
\hline 220 & $\mathbf{3 8 3 2}$ & $\mathbf{B}$ & $\begin{array}{l}\text { Dispatch: 13/8/2020 } \\
\text { Total pages: 46 } \\
\text { Disk Received } \\
\text { Disk Used } \square\end{array}$ & $\begin{array}{l}\text { Journal: Commun. Math. Phys. } \\
\text { Not Used } \square \\
\text { Corrupted } \square \\
\text { Mismatch } \square\end{array}$ \\
\hline Jour. No & Ms. No.
\end{tabular}


To start, we provide a more convenient expression for the exponents $\beta_{k}(\varepsilon)$, which shows that the smallest ones are $\mathcal{O}\left(\varepsilon^{-1 / 6}\right)$. Indeed, we deduce from (73) that we can write

$$
\beta_{k}(\varepsilon)=\frac{C_{0}}{\varepsilon^{1 / 6}} g_{k}(\varepsilon), \quad C_{0}:=\frac{3}{2}\left(\pi \rho^{2} \gamma^{*}\right)^{1 / 3},
$$

where for any given $k$ we introduce the function

$$
g_{k}(\varepsilon):=\frac{\tilde{\gamma}_{k}^{1 / 3}}{3}\left[2\left(\frac{\varepsilon}{\varepsilon_{k}}\right)^{1 / 6}+\left(\frac{\varepsilon_{k}}{\varepsilon}\right)^{1 / 3}\right], \quad \varepsilon_{k}:=\frac{D_{0} \tilde{\gamma}_{k}^{2}}{|k|^{6}}, \quad D_{0}:=\left(\frac{\pi \gamma^{*}}{\rho}\right)^{2} .
$$

It is straightforward to check that each function $g_{k}(\varepsilon)$ attains its minimum at $\varepsilon=\varepsilon_{k}$, with the (positive) minimum value $g_{k}\left(\varepsilon_{k}\right)=\tilde{\gamma}_{k}^{1 / 3}$. Recall that the constant $\gamma^{*}=\gamma_{\hat{q}}^{*}$ and the normalized numerators $\tilde{\gamma}_{k}=\gamma_{k} / \gamma^{*}$ were introduced in (60) and (62), respectively.

Since we are interested in obtaining asymptotic estimates for the splitting distance, rather than lower bounds, we need to determine for any given $\varepsilon$ the most dominant harmonic, which is given by the smallest value $g_{k}(\varepsilon)$, reached for some integer vector $k=S_{1}(\varepsilon)$ to be determined. In fact, as in [DGG16] we may replace, for $\varepsilon$ small, the functions $g_{k}(\varepsilon)$ by approximations $g_{k}^{*}(\varepsilon)$, obtained by neglecting the asymptotic terms going to 0 in Proposition 11. More precisely, for $k=s(q, n)$ belonging to a concrete resonant sequence, we use the approximations (54-55) for $|s(q, n)|$ and $\gamma_{s(q, n)}$ as $n \rightarrow \infty$, given in Proposition 11, and we obtain the following approximations:

$$
\begin{aligned}
g_{s(q, n)}(\varepsilon) & \approx g_{s(q, n)}^{*}(\varepsilon):=\frac{\left(\tilde{\gamma}_{q}^{*} b_{s(q, n)}\right)^{1 / 3}}{3}\left[2\left(\frac{\varepsilon}{\varepsilon_{s(q, n)}^{*}}\right)^{1 / 6}+\left(\frac{\varepsilon_{s(q, n)}^{*}}{\varepsilon}\right)^{1 / 3}\right], \\
\varepsilon_{s(q, n)} & \approx \varepsilon_{s(q, n)}^{*}:=\frac{D_{0}\left(\tilde{\gamma}_{q}^{*}\right)^{2}}{K_{q}^{3} b_{s(q, n)} \cdot \lambda^{3 n}},
\end{aligned}
$$

with the oscillating factors $b_{s(q, n)}$ introduced in (56). Notice that each function $g_{s(q, n)}^{*}(\varepsilon)$ has its minimum at $\varepsilon_{s(q, n)}^{*}$, whose dependence on $n$ is not strictly geometric (decreasing with ratio $\lambda^{3}$ ), but "perturbed" by the oscillating factor $b_{s(q, n)}$. Analogously, the minimum values $g_{s(q, n)}^{*}\left(\varepsilon_{s(q, n)}^{*}\right)=\tilde{\gamma}_{q}^{*} b_{s(q, n)}$ are not constant but oscillating. The size of such "perturbations" is given by the value $\delta$ introduced in (48).

Remark 14. The most dominant harmonic cannot be found in a non-essential resonant sequence. Indeed, if $s(q, n)=c s\left(\bar{q}, n_{0}+n\right)$ with $|c|>1$ and $n_{0} \geq 0$, then $g_{s(q, n)}^{*}(\varepsilon)=$ $|c| g_{s\left(\bar{q}, n_{0}+n\right)}^{*}(\varepsilon)$ (see also Remark 13(b)).

The sequence of primary resonances $s_{0}(n)=s(\widehat{q}, n)$, defined in (59), plays an important role since it gives the smallest minimum values among the functions $g_{k}^{*}(\varepsilon)$, and hence they will provide the most dominant harmonics, at least for $\varepsilon$ close to such minima. With this fact in mind, and recalling that $\tilde{\gamma}_{\widehat{q}}^{*}=1$, we introduce

$$
\begin{aligned}
\bar{g}_{n}(\varepsilon) & :=g_{s_{0}(n)}^{*}(\varepsilon)=\frac{\bar{b}_{n}^{1 / 3}}{3}\left[2\left(\frac{\varepsilon}{\bar{\varepsilon}_{n}}\right)^{1 / 6}+\left(\frac{\bar{\varepsilon}_{n}}{\varepsilon}\right)^{1 / 3}\right], \\
\bar{\varepsilon}_{n} & :=\varepsilon_{s_{0}(n)}^{*}=\frac{D_{0}}{K_{\widehat{q}}^{3} \bar{b}_{n} \cdot \lambda^{3 n}}, \\
\bar{b}_{n} & :=b_{s_{0}(n)}=1+\delta \cos \left(2 \pi \cdot n \phi+2 \psi_{\widehat{q}}-\theta\right),
\end{aligned}
$$

\begin{tabular}{|l|l|l|l|l|l|}
\hline 220 & $\mathbf{3 8 3 2}$ & $\mathbf{B}$ & $\begin{array}{l}\text { Dispatch: 13/8/2020 } \\
\text { Total pages: 46 } \\
\text { Disk Received } \\
\text { Disk Used } \square\end{array}$ & $\begin{array}{l}\text { Journal: Commun. Math. Phys. } \\
\text { Not Used } \square \\
\text { Corrupted } \square \\
\text { Mismatch } \square\end{array}$ \\
\hline
\end{tabular}


where the constants $\phi, \theta, \delta, \psi_{\widehat{q}}, K_{\widehat{q}}$ and $D_{0}$ are introduced in (37), (47-48), (52-53) and (75), respectively.

In order to determine the most dominant harmonic for any given $\varepsilon$, we have to study the relative position of the functions $g_{s(q, n)}^{*}(\varepsilon)$ and the intersections between their graphs. Due to the (essentially) geometric behavior of the minima $\varepsilon_{s(q, n)}^{*}$ as $n \rightarrow \infty$, it is convenient to replace $\varepsilon$ by a $\log$ arithmic variable:

$$
\zeta=\operatorname{Lg} \frac{D_{0}}{K_{\widehat{q}}^{3}}-\operatorname{Lg} \varepsilon, \quad \text { i.e. } \varepsilon=\frac{D_{0}}{K_{\widehat{q}}^{3} \lambda^{3 \zeta}}
$$

(notice that $\zeta \rightarrow \infty$ as $\varepsilon \rightarrow 0^{+}$), where we introduce the notation

$$
\operatorname{Lg} x:=\log _{\left(\lambda^{3}\right)} x=\frac{\ln x}{3 \ln \lambda} .
$$

We define for any given $Z \in \mathbb{R}$ and $Y>0$ the following "hyperbolic cosine-like" function:

$$
\mathcal{C}(\zeta ; Z, Y):=Y^{1 / 3} \mathcal{C}_{0}(\zeta-Z), \quad \mathcal{C}_{0}(\zeta):=\frac{1}{3}\left(2 \lambda^{-\zeta / 2}+\lambda^{\zeta}\right)
$$

Any function $\mathcal{C}(\zeta ; Z, Y)$ has its minimum at $\zeta=Z$ with $\mathcal{C}(Z ; Z, Y)=Y^{1 / 3}$ as the minimum value, and is a convex function. In fact, the point $\left(Z, Y^{1 / 3}\right)$ of its graph determines the function, and the graph becomes divided at this point into a "decreasing branch" $(\zeta<Z)$ and an "increasing branch" $(\zeta>Z)$.

Translating definitions (76-79) of $g_{s(q, n)}^{*}(\varepsilon), \varepsilon_{s(q, n)}^{*}, \bar{g}_{n}(\varepsilon), \bar{\varepsilon}_{n}$ into the new variable, we get:

$$
\begin{aligned}
f_{s(q, n)}^{*}(\zeta) & :=\mathcal{C}\left(\zeta ; \zeta_{s(q, n)}^{*}, \tilde{\gamma}_{q}^{*} b_{s(q, n)}\right), \\
\zeta_{s(q, n)}^{*} & :=n+3 \operatorname{Lg} \frac{K_{q}}{K_{\widehat{q}}}-2 \operatorname{Lg} \tilde{\gamma}_{q}^{*}+\operatorname{Lg} b_{s(q, n)}, \\
\bar{f}_{n}(\zeta) & :=\mathcal{C}\left(\zeta ; \bar{\zeta}_{n}, \bar{b}_{n}\right) . \quad \bar{\zeta}_{n}:=n+\operatorname{Lg} \bar{b}_{n} .
\end{aligned}
$$

Notice that, if the oscillating terms $b_{s(q, n)}$ are not taken into account (i.e. if we assume $\delta=$ 0 in (48)), the graph of a function $f_{s(q, n+1)}^{*}$ is a translation of $f_{s(q, n)}^{*}$ to distance 1 , which would be the situation for the case of quadratic frequencies considered in [DGG16]. What we actually have for cubic frequencies is an $\mathcal{O}(\delta)$-perturbation of this situation, due to the terms $b_{s}(q, n)$ defined in (56).

Remark 15. In fact, if analogous computations are carried out for the quadratic case, the function $\mathcal{C}_{0}(\zeta)$ introduced in (82) should be replaced by an expression of the type $\left(\lambda^{-\zeta}+\lambda^{\zeta}\right) / 2=\cosh (\zeta \ln \lambda)$ (with a somewhat different definition of the variable $\zeta$ ). An expression of this type in asymptotic estimates for the splitting appeared for the first time in [DGJS97] (see also [DG04]). We point out that our "hyperbolic cosine-like" function $\mathcal{C}_{0}(\zeta)$ is no longer an even function of $\zeta$ in the cubic case considered here, according to the definition (82). In other words, the symmetry of the "true" hyperbolic $\operatorname{cosine}$ function $\cosh (\zeta \ln \lambda)$ between the decreasing and increasing branches, that takes place in the quadratic case, is not preserved in the cubic case.

\begin{tabular}{|l|l|l|l|l|l|}
\hline 220 & $\mathbf{3 8 3 2}$ & $\mathbf{B}$ & $\begin{array}{l}\text { Dispatch: 13/8/2020 } \\
\text { Total pages: 46 } \\
\text { Disk Received } \\
\text { Disk Used } \square\end{array}$ & $\begin{array}{l}\text { Journal: Commun. Math. Phys. } \\
\text { Not Used } \\
\text { Corrupted } \\
\text { Mismatch } \square\end{array}$ \\
\hline Jour. No & Ms. No.
\end{tabular}


In order to study the dependence of the most dominant harmonics on $\varepsilon$, now replaced by the logarithmic variable $\zeta$ introduced in (81), it is useful to consider the intersections between the graphs of functions (83), since this gives the values of $\zeta$ at which a change in the dominance may take place. The next two lemmas show that, if we consider the graphs associated to the functions $f_{k}^{*}(\zeta)$ and $f_{\bar{k}}^{*}(\zeta)$ associated to different quasi-resonances $k, \bar{k}$, only two situations are possible: they do not intersect (which says that one of them always dominates the other one), or they intersect transversely at a unique point (and in this case a unique change in the dominance takes place among such two quasi-resonances). Namely, in Lemma 16 we show that $f_{k}^{*}$ and $f_{\bar{k}}^{*}$ cannot be the same function, and in Lemma 17 (formulated, by convenience, in terms of the functions introduced in (82)) we provide the condition for the existence of intersection between their graphs, as well as an explicit formula for this intersection, and some additional bounds to be used later. Recall that the sets $\mathcal{Z}$ and $\mathcal{A}$ are defined in (10) and (38).

Lemma 16. For any given $k, \bar{k} \in \mathcal{A} \cap \mathcal{Z}$ with $k \neq \bar{k}$, the functions $f_{k}^{*}(\zeta)$ and $f_{\bar{k}}^{*}(\zeta)$ do not coincide.

Proof. Recalling the definition (45), let us write $k=s(q, n)$ and $\bar{k}=s(\bar{q}, \bar{n})$. If $f_{k}^{*}=$ $f_{\bar{k}}^{*}$, then we have $g_{k}^{*}=g_{\bar{k}}^{*}$ and, by definition (76), we get $\tilde{\gamma}_{q}^{*} b_{k}=\tilde{\gamma}_{\bar{q}}^{*} b_{\bar{k}}$ and $\varepsilon_{k}^{*}=\varepsilon_{\bar{k}}^{*}$. By (53), such two equalities can be rewritten as $\left|r_{q}\right| K_{q} b_{k}=\left|r_{\bar{q}}\right| K_{\bar{q}} b_{\bar{k}}$ and $K_{q} b_{k} \lambda^{n}=$ $K_{\bar{q}} b_{\bar{k}} \lambda^{\bar{n}}$, respectively. We deduce that the small divisors (40) satisfy $\left|r_{\bar{q}} / r_{q}\right|=\lambda^{\bar{n}-n}$ but, from the fundamental property (43), we have $\left|r_{q}\right|,\left|r_{\bar{q}}\right| \in(1 / 2 \lambda, 1 / 2)$. This says that $n=\bar{n}$ and hence $\left|r_{q}\right|=\left|r_{\bar{q}}\right|$, but from definition (40) and the fact that $\omega$ is a nonresonant vector we deduce that $q= \pm \bar{q}$, which contradicts the assumption $k \neq \bar{k}$ (recall that $k, \bar{k} \in \mathcal{Z})$.

Lemma 17. Let $Z_{1}, Z_{2} \in \mathbb{R}$ and $Y_{1}, Y_{2}>0$ with $\left(Z_{1}, Y_{1}\right) \neq\left(Z_{2}, Y_{2}\right)$, and define

$$
Z=Z_{2}-Z_{1}, \quad W=\left(\frac{Y_{2}}{Y_{1}}\right)^{1 / 3} .
$$

Then, we have:

(a) The graphs of the functions $\mathcal{C}\left(\zeta ; Z_{1}, Y_{1}\right)$ and $\mathcal{C}\left(\zeta ; Z_{2}, Y_{2}\right)$ intersect if and only if $\lambda^{Z}<\min \left(W, W^{-2}\right)$ or $\lambda^{Z}>\max \left(W, W^{-2}\right)$. If so, the intersection is unique and transverse, and takes place at the point given by

$$
\zeta^{*}=Z_{1}+2 \operatorname{Lg} \frac{2 \lambda^{Z}\left(W \lambda^{Z / 2}-1\right)}{\lambda^{Z}-W} .
$$

(b) The following upper/lower bound holds:

$$
\begin{array}{ll}
\zeta^{*}<Z_{1}+2 \operatorname{Lg} \frac{2 \lambda^{Z}}{W-\lambda^{Z}} & \text { if } \lambda^{Z}<\min \left(W, W^{-2}\right), \\
\zeta^{*}>Z_{1}+2 \operatorname{Lg} 2\left(W \lambda^{Z / 2}-1\right) & \text { if } \lambda^{Z}>\max \left(W, W^{-2}\right) .
\end{array}
$$

Proof. Introducing the variable $\xi=\zeta-Z_{1}$, we see from definition (82) that the intersection between the graphs of $\mathcal{C}\left(\zeta ; Z_{1}, Y_{1}\right)$ and $\mathcal{C}\left(\zeta ; Z_{2}, Y_{2}\right)$ corresponds to the solution of the equation $\mathcal{C}_{0}(\xi)=W \mathcal{C}_{0}(\xi-Z)$, where we have $(Z, W) \neq(0,1)$. After some straightforward computations, we see that this solution $\xi=\xi^{*}$ is given by

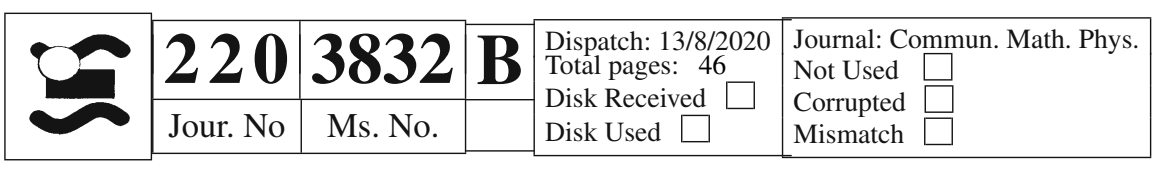


$\lambda^{3 \xi^{*} / 2}=\frac{2 \lambda^{Z}\left(W \lambda^{Z / 2}-1\right)}{\lambda^{Z}-W}$, which leads directly to the formula (86) for $\zeta^{*}=Z_{1}+\xi^{*}$. Notice that the intersection does not take place if $\lambda^{Z}$ belongs to the interval of endpoints $W$ and $W^{-2}$ (indeed, in this case the numerator and denominator in the expression (86) would have different sign).

To complete the proof of (a), we have to show the transversality of the intersection. This amounts to see that the solution obtained above does not satisfy the equation $\mathcal{C}_{0}^{\prime}\left(\xi^{*}\right)=W \mathcal{C}_{0}^{\prime}\left(\xi^{*}-Z\right)$. Indeed, solving this new equation we get $\lambda^{3 \xi^{*} / 2}=$ $\frac{\lambda^{Z}\left(W \lambda^{Z / 2}-1\right)}{W-\lambda^{Z}}$, which is possible only if $\lambda^{Z}$ does belong to the interval of endpoints $W$ and $W^{-2}$ (the case excluded above).

The proof of the bound (b) for $\zeta^{*}$, in the two cases considered, is straightforward from the formula (86).

3.2. Estimate of the most dominant harmonic. We introduce the positive function $h_{1}(\varepsilon)$ appearing in the exponent in Theorem 1 as the minimum, for any given $\varepsilon$, of the values $g_{k}^{*}(\varepsilon)$ among the quasi-resonances, and we denote $S_{1}=S_{1}(\varepsilon)$ the integer vector $k$ at which such minimum is reached:

$$
h_{1}(\varepsilon):=\min _{k \in \mathcal{A}} g_{k}^{*}(\varepsilon)=g_{S_{1}}^{*}(\varepsilon) .
$$

In fact, by Remark 14 the integer vector providing the minimum is always an essential quasi-resonance: $S_{1}(\varepsilon) \in \mathcal{A}_{0}$.

Our aim is to study some of the properties of $h_{1}(\varepsilon)$, putting emphasis on the dependence of such functions on the arithmetic properties of the cubic frequency vector $\omega$, studied in Sect. 2. Namely, we prove that the function $h_{1}(\varepsilon)$ satisfies the following properties:

- It is piecewise-smooth and piecewise-convex (and continuous), with corners (i.e. jump discontinuities of the derivative) associated to changes in the dominant harmonic (i.e. discontinuities of the "piecewise-constant" function $S_{1}(\varepsilon)$ ).

- It is bounded, providing (positive) lower and upper bounds for it.

- It is quasiperiodic (and not periodic) with respect to $\ln \varepsilon$, with two frequencies whose ratio is the irrational number $\phi$ defined in (37).

As in Sect. 3.1, we can translate the function $h_{1}(\varepsilon)$ into the logarithmic variable $\zeta$ introduced in (81):

$$
F_{1}(\zeta):=\min _{k \in \mathcal{A}} f_{k}^{*}(\zeta)=f_{R_{1}}^{*}(\zeta)
$$

with $R_{1}=R_{1}(\zeta)=S_{1}(\varepsilon)$. We also define an analogous but somewhat simpler function, taking into account only the primary resonances $s_{0}(n)$ introduced in (59) and involved in (80) and (85):

$$
\bar{F}_{1}(\zeta):=\min _{n \geq 0} \bar{f}_{n}(\zeta)=\bar{f}_{N_{1}}(\zeta)
$$

with $N_{1}=N_{1}(\zeta)$. In other words, the most dominant harmonic among the primary resonances corresponds to $\bar{R}_{1}=\bar{R}_{1}(\zeta)=s_{0}\left(N_{1}\right)$.

\begin{tabular}{|l|l|l|l|l|l|}
\hline $\mathbf{2} 2 \mathbf{2 0}$ & $\mathbf{3 8 3 2}$ & $\mathbf{B}$ & $\begin{array}{l}\text { Dispatch: 13/8/2020 } \\
\text { Total pages: 46 } \\
\text { Disk Received } \\
\text { Disk Used } \square\end{array}$ & $\begin{array}{l}\text { Journal: Commun. Math. Phys. } \\
\text { Not Used } \square \\
\text { Corrupted } \\
\text { Mismatch } \square\end{array}$ \\
\hline
\end{tabular}


Clearly, for any $\zeta$ we have

$$
F_{1}(\zeta) \leq \bar{F}_{1}(\zeta)
$$

In order to provide an accurate description of the splitting, it is useful to study whether the equality between the above functions can be established for any value of $\zeta$, or there exist some intervals of $\zeta$ where it does not hold. This amounts to study whether the dominant harmonics can always be found among the primary resonances $\left(R_{1}=\bar{R}_{1}\right)$ or, on the contrary, secondary resonances have to be taken into account (and in this case the function $F_{1}(\zeta)$ is somewhat more complicated). Such two possiblities also take place in the quadratic case considered in [DGG16].

We can provide an alternative definition for $F_{1}(\zeta)$ as the minimum of the following functions, associated to any given resonant sequence $s(q, n)$ :

$$
\widetilde{F}_{1}^{(q)}(\zeta):=\min _{n \geq 0} f_{s(q, n)}^{*}(\zeta)
$$

(for the primary resonances, we have $\widetilde{F}_{1}^{(\widehat{q})}=\bar{F}_{1}$ ). Clearly, it is enough to consider essential primitives $\left(q \in \mathcal{P}_{0}\right)$, and hence we can write

$$
F_{1}(\zeta)=\min _{q \in \mathcal{P}_{0}} \widetilde{F}_{1}^{(q)}(\zeta)
$$

Such functions $\widetilde{F}_{1}^{(q)}(\zeta)$ are completely analogous to $\bar{F}_{1}(\zeta)$. We are going to study only the function $\bar{F}_{1}(\zeta)$, showing that it is quasiperiodic and providing lower and upper bounds for it, and the same will hold for $\widetilde{F}_{1}^{(q)}(\zeta)$, with the bounds multiplied by the factor $\left(\tilde{\gamma}_{q}^{*}\right)^{1 / 3} \geq 1$ in view of (83). Notice also that only a finite number of primitives $q$ are involved in (91), due to the fact that the (normalized) limits $\tilde{\gamma}_{q}^{*}$ have the lower bound (58), which is increasing with respect to $|q|$.

Remark 18. Although we implicitly assume that there exists only one sequence of primary resonances (see Remark 13(a)), it is not hard to adapt our definitions and results to the case of two or more sequences of primary resonances. In this case, we would choose in (59) one of such sequences as "the" sequence $s_{0}(n)$, when the functions $\bar{g}_{n}(\varepsilon)$ and $\bar{f}_{n}(\zeta)$ are defined in (78) and (85) (see also [DGG16]).

Now we proceed to study the function $\bar{F}_{1}(\zeta)$ introduced in (88). Notice that we can regard this function as an $\mathcal{O}(\delta)$-perturbation of the function obtained if we had $\delta=0$ in (48) (and hence $\bar{b}_{n}=1$ in (80)). Of course, this is fictitious since $\delta$ is determined by the frequency vector $\omega$ and is not a true parameter. With this in mind, we define "unperturbed" functions

$$
\begin{aligned}
& \bar{f}_{n}^{(0)}(\zeta):=\mathcal{C}(\zeta ; n, 1)=\mathcal{C}_{0}(\zeta-n) \\
& \bar{F}_{1}^{(0)}(\zeta):=\min _{n} \bar{f}_{n}^{(0)}(\zeta)=\bar{f}_{N_{1}^{(0)}}^{(0)}(\zeta)
\end{aligned}
$$

The index $N_{1}^{(0)}=N_{1}^{(0)}(\zeta)$ providing the minimum can easily be determined. On one hand, we use that each function $\bar{f}_{n}^{(0)}(\zeta)$ reaches it minimum at $\zeta_{n}=n$. On the other hand, applying Lemma 17 (a) (with $Z=1$ and $W=1$ ) we find its corners, given by

\begin{tabular}{|l|l|l|l|l|l|}
\hline 220 & $\mathbf{3 8 3 2}$ & $\mathbf{B}$ & $\begin{array}{l}\text { Dispatch: 13/8/2020 } \\
\text { Total pages: 46 } \\
\text { Disk Received } \\
\text { Disk Used } \square\end{array}$ & $\begin{array}{l}\text { Journal: Commun. Math. Phys. } \\
\text { Not Used } \square \\
\text { Corrupted } \square \\
\text { Mismatch } \square\end{array}$ \\
\hline Jour. No & Ms. No.
\end{tabular}


the (transverse) intersection between the graphs of consecutive functions $\bar{f}_{n}^{(0)}(\zeta)$ and $\bar{f}_{n+1}^{(0)}(\zeta)$ :

$$
\zeta_{n}^{\prime}:=n+\xi_{0}, \quad \xi_{0}:=2 \operatorname{Lg} \frac{2 \lambda}{\sqrt{\lambda}+1}, \quad \text { i.e. } \quad \lambda^{3 \xi_{0} / 2}=\frac{2 \lambda}{\sqrt{\lambda}+1} .
$$

Hence, we can write $\xi_{0}=\xi_{0}(\omega)$ and, using that $\lambda>1$, it is not hard to see that $1 / 3<$ $\xi_{0}<1 / 2$ (see in Sect. 3.4 the concrete value for the case of the cubic golden vector). Introducing the intervals $\mathcal{I}_{n}:=\left[\zeta_{n-1}^{\prime}, \zeta_{n}^{\prime}\right]$, we see that $N_{1}^{(0)}(\zeta)=n$ for any $\zeta \in \mathcal{I}_{n}$ (strictly speaking, there are two possible values at the endpoints $\zeta_{n}^{\prime}$ of the intervals). In this way, the function $N_{1}^{(0)}(\zeta)$ is "piecewise-constant" with jump discontinuities at the points $\zeta_{n}^{\prime}$, and the function $\bar{F}_{1}^{(0)}(\zeta)$ is 1-periodic, continuous and piecewise-smooth with corners at the same points $\zeta_{n}^{\prime}$. We also obtain the following extreme values:

$$
\begin{aligned}
\min \bar{F}_{1}^{(0)}(\zeta) & =\bar{F}_{1}^{(0)}(n)=\mathcal{C}_{0}(0)=1 \\
\max \bar{F}_{1}^{(0)}(\zeta) & =\bar{F}_{1}^{(0)}\left(\zeta_{n}^{\prime}\right)=\mathcal{C}_{0}\left(\xi_{0}\right)=\mathcal{C}_{0}\left(\xi_{0}-1\right) \\
& =J_{1}^{(0)}=J_{1}^{(0)}(\omega):=\frac{1}{3}\left[2\left(\frac{\sqrt{\lambda}+1}{2 \lambda}\right)^{1 / 3}+\left(\frac{2 \lambda}{\sqrt{\lambda}+1}\right)^{2 / 3}\right] .
\end{aligned}
$$

Returning to the "perturbed" function $\bar{F}_{1}(\zeta)$, the next lemma shows that, for any $\zeta$, the index $N_{1}(\zeta)$ providing the minimum in definition (88), can be found among a finite number (not depending on $\zeta$ ) of values around $N_{1}^{(0)}(\zeta)$.

Lemma 19. For any $\zeta$, we have $N_{1}^{(0)}(\zeta)-N^{-} \leq N_{1}(\zeta) \leq N_{1}^{(0)}(\zeta)+N^{+}$, where we define

$$
\begin{aligned}
& N^{-}=N^{-}(\omega):=\log _{\lambda}\left[\max \left(\frac{1+\delta}{1-\delta}, 2(1+\delta)^{1 / 2} \lambda^{3\left(1-\xi_{0}\right) / 2}+1\right)\right], \\
& N^{+}=N^{+}(\omega):=\log _{\lambda}\left[\max \left(\frac{1+\delta}{1-\delta},\left(\frac{\lambda^{3 \xi_{0} / 2}+2(1+\delta)^{1 / 2}}{2(1-\delta)^{1 / 2}}\right)^{2}\right)\right] .
\end{aligned}
$$

Proof. Let us assume that $\zeta$ belongs to a concrete interval $\mathcal{I}_{n}$, where we have $N_{1}^{(0)}(\zeta)=$ $n$. In order to show that $N_{1}(\zeta)$ belongs to the interval $\left[n-N^{-}, n+N^{+}\right]$, we have to show that, for any $m$ not belonging to this interval, we have

$$
\bar{f}_{m}(\zeta)>\bar{f}_{n}(\zeta) \text { for any } \zeta \in \mathcal{I}_{n}
$$

To study the relative position of the functions $\bar{f}_{n}(\zeta)$ and $\bar{f}_{m}(\zeta)$ (defined in (85)), we will apply Lemma 17 showing that their graphs do intersect at a point $\zeta_{n, m}^{*}$, which satisfies:

$$
\begin{array}{ll}
\zeta_{n, m}^{*}<\zeta_{n-1}^{\prime} & \text { if } m-n<-N^{-}, \\
\zeta_{n, m}^{*}>\zeta_{n}^{\prime} & \text { if } m-n>N^{+},
\end{array}
$$

which says that the (unique) intersection takes place outside the interval $\mathcal{I}_{n}$, and implies the inequality (96).

\begin{tabular}{|l|l|l|l|l|l|}
\hline $\mathbf{2} 2 \mathbf{2 0}$ & $\mathbf{3 8 3 2}$ & $\mathbf{B}$ & $\begin{array}{l}\text { Dispatch: 13/8/2020 } \\
\text { Total pages: 46 } \\
\text { Disk Received } \\
\text { Disk Used } \square\end{array}$ & $\begin{array}{l}\text { Journal: Commun. Math. Phys. } \\
\text { Not Used } \square \\
\text { Corrupted } \square \\
\text { Mismatch } \square\end{array}$ \\
\hline
\end{tabular}


In order to apply Lemma 17 , we consider the values $Z=\bar{\zeta}_{m}-\bar{\zeta}_{n}$ and $W=$ $\left(\bar{b}_{m} / \bar{b}_{n}\right)^{1 / 3}$, which satisfy the equality

$$
\lambda^{Z}=W \lambda^{m-n}
$$

On the other hand, recalling that $\bar{b}_{n}, \bar{b}_{m} \in[1-\delta, 1+\delta]$, we have $\left(\frac{1-\delta}{1+\delta}\right)^{1 / 3} \leq W \leq$ $\left(\frac{1+\delta}{1-\delta}\right)^{1 / 3}$

To prove the first assertion of (97), we use the first bound of Lemma 17(b), which reads

$$
\zeta_{n, m}^{*}<\bar{\zeta}_{n}+2 \operatorname{Lg} \frac{2 \lambda^{m-n}}{1-\lambda^{m-n}} \quad \text { if } \quad \lambda^{m-n}<\min \left(1, W^{-3}\right),
$$

where we the equality (98) has been taken into account. By the definition of $N^{-}$, it is clear that $\lambda^{n-m}>\frac{1+\delta}{1-\delta} \geq\left(\min \left(1, W^{-3}\right)\right)^{-1}$. Moreover, the inequality $\zeta_{n, m}^{*}<\zeta_{n-1}^{\prime}$ holds provided

$$
\operatorname{Lg} \bar{b}_{n}+2 \operatorname{Lg} \frac{2 \lambda^{m-n}}{1-\lambda^{m-n}} \leq-1+\xi_{0}
$$

Replacing $\bar{b}_{n}$ by $1+\delta$, the subsequent inequality can be rewritten as

$$
\lambda^{n-m} \geq 2(1+\delta)^{1 / 2} \lambda^{3\left(1-\xi_{0}\right) / 2}+1,
$$

also included in the definition of $N^{-}$, which completes the proof of the first assertion of (97).

For the second assertion of (97) we can proceed in similar terms, using the second bound of Lemma 17(b). Nevertheless, the associated computations are somewhat different due to the lack of symmetry of the functions $\bar{f}_{n}(\zeta)$ in the cubic case (see Remark 15). We omit the details.

In the following proposition, we provide a lower and an upper bound for the functions $\bar{F}_{1}(\zeta)$ and $F_{1}(\zeta)$, and hence for $h_{1}(\varepsilon)$, as $\mathcal{O}(\delta)$-perturbations of the values obtained in (94-95). More precisely, such bounds will be given by the values

$$
J_{0}^{-}=J_{0}^{-}(\omega):=(1-\delta)^{1 / 3}, \quad J_{1}^{+}=J_{1}^{+}(\omega):=J_{1}^{(0)}(1+\delta)^{1 / 3},
$$

which satisfy $0<J_{0}^{-}<1<J_{1}^{(0)}<J_{1}^{+}$. Recall that lower and an upper bounds for $h_{1}(\varepsilon)$ or, equivalently, for $F_{1}(\zeta)$, can be associated to upper and lower bounds for the splitting distance, respectively (see also [DGG14a]). Recalling the value $B_{0}^{-}=B_{0}^{-}(\omega)$ defined in (65), we also introduce the "strong separation condition":

$$
B_{0}^{-} \geq J_{1}^{+},
$$

which is somewhat more restrictive than the "weak separation condition" introduced in (66). Under the strong condition, the inequality (89) becomes an equality, i.e. the dominant harmonic is always given by a primary resonance, and hence the function $F_{1}(\zeta)=h_{1}(\varepsilon)$ becomes somewhat simpler. Such a condition is fulfilled for the cubic golden frequency vector, as we show in Sect. 3.4.

\begin{tabular}{|l|l|l|l|l|l|}
\hline 220 & $\mathbf{3 8 3 2}$ & $\mathbf{B}$ & $\begin{array}{l}\text { Dispatch: 13/8/2020 } \\
\text { Total pages: 46 } \\
\text { Disk Received } \\
\text { Disk Used } \square\end{array}$ & $\begin{array}{l}\text { Journal: Commun. Math. Phys. } \\
\text { Not Used } \square \\
\text { Corrupted } \square \\
\text { Mismatch } \square\end{array}$ \\
\hline Jour. No & Ms. No.
\end{tabular}


Proposition 20. The functions $F_{1}(\zeta)$ and $\bar{F}_{1}(\zeta)$ are positive, continuous and piecewisesmooth, and satisfy for any $\zeta$ the bounds:

$$
J_{0}^{-} \leq F_{1}(\zeta) \leq \bar{F}_{1}(\zeta) \leq J_{1}^{+}
$$

with $J_{0}^{-}$and $J_{1}^{+}$defined in (99). Moreover, if the strong separation condition (100) is fulfilled, then we have $F_{1}(\zeta)=\bar{F}_{1}(\zeta)$ for any $\zeta$, and hence the most dominant harmonic is always given by a primary resonance.

Proof. The lower bound for $F_{1}(\zeta)$ is a direct consequence of (90-91), using that for any $k=s(q, n) \in \mathcal{A}$ we have the lower bound

$$
f_{s(q, n)}^{*}(\zeta) \geq\left(\tilde{\gamma}_{q}^{*} b_{s(q, n)}\right)^{1 / 3} \geq(1-\delta)^{1 / 3}
$$

which comes from (83), using also that $b_{s}(q, n) \geq 1-\delta$ by (56).

To provide an upper bound for $\bar{F}_{1}(\zeta)$, we take into account that $\bar{b}_{n} \leq 1+\delta$ and introduce the function

$$
\bar{F}_{1}^{+}(\zeta):=\min _{n \geq 0} \bar{f}_{n}^{+}(\zeta), \quad \bar{f}_{n}^{+}(\zeta):=\mathcal{C}\left(\zeta ; \bar{\zeta}_{n}^{+}, 1+\delta\right), \quad \bar{\zeta}_{n}^{+}:=n+\operatorname{Lg}(1+\delta),
$$

defined as in (88) but replacing $\bar{b}_{n}$ by $1+\delta$ in (85). Notice that the function $\bar{F}_{1}^{+}(\zeta)$ can easily be related to the "unperturbed" function defined in (92): for any $\zeta$, we have

$$
\bar{F}_{1}^{+}(\zeta)=(1+\delta)^{1 / 3} \bar{F}_{1}^{(0)}(\zeta-\operatorname{Lg}(1+\delta))
$$

and we deduce from (95) and (99) that $\max \bar{F}_{1}^{+}(\zeta)=J_{1}^{+}$.

We study the relative position of the graphs of the functions $\bar{f}_{n}(\zeta)$ and $\bar{f}_{n}^{+}(\zeta)$ by applying Lemma $17(\mathrm{a})$, with $Z=\bar{\zeta}_{n}^{+}-\bar{\zeta}_{n}=\operatorname{Lg}\left((1+\delta) / \bar{b}_{n}\right)$ and $W=\left((1+\delta) / \bar{b}_{n}\right)^{1 / 3}$. In general we have $\bar{b}_{n}<1+\delta$ and, since $\lambda^{Z}=W$, the graphs do not intersect and we have $\bar{f}_{n}(\zeta)<\bar{f}_{n}^{+}(\zeta)$ for any $\zeta$. Instead, if $\bar{b}_{n}=1+\delta$ (a rather particular case) then the two functions obviously coincide. We deduce, for any $\zeta$, the bound

$$
\bar{F}_{1}(\zeta) \leq \bar{F}_{1}^{+}(\zeta) \leq J_{1}^{+}
$$

Finally, to show that the strong separation condition (100) implies the equality $F_{1}(\zeta)=\bar{F}_{1}(\zeta)$, it is enough to see that a lower bound for the functions $\widetilde{F}_{1}^{(q)}(\zeta)$ introduced in (90), for $q \neq \widehat{q}$, is greater than the upper bound $J_{1}^{+}$for $\bar{F}_{1}(\zeta)$, obtained above. Indeed, for secondary resonances $s(q, n)$, with $q \neq \widehat{q}$, the lower bound (101) becomes

$$
f_{s(q, n)}^{*}(\zeta) \geq\left(\tilde{\gamma}_{\widehat{q}^{\prime}}^{*}(1-\delta)\right)^{1 / 3}=B_{0}^{-} \geq J_{1}^{+},
$$

where $\gamma_{\widehat{q}}^{*}$, is the minimum of the "mean Diophantine constants" for secondary resonances (see (63)), and the same lower bound holds for the functions $\widetilde{F}_{1}^{(q)}(\zeta), q \neq \widehat{q}$.

\begin{tabular}{|l|l|l|l|l|l|}
\hline $\mathbf{2} 2 \mathbf{2 0}$ & $\mathbf{3 8 3 2}$ & $\mathbf{B}$ & $\begin{array}{l}\text { Dispatch: 13/8/2020 } \\
\text { Total pages: 46 } \\
\text { Disk Received } \\
\text { Disk Used } \square\end{array}$ & $\begin{array}{l}\text { Journal: Commun. Math. Phys. } \\
\text { Not Used } \square \\
\text { Corrupted } \\
\text { Mismatch } \square\end{array}$ \\
\hline
\end{tabular}


Remark 21. It is an interesting question whether the lower and upper bounds $J_{0}^{-}$and $J_{1}^{+}$provided by this proposition are sharp, i.e. they coincide with the infimum and the supremum of the function $F_{1}(\zeta)$. On one hand, we can expect the lower bound $J_{0}^{-}$ (and hence the upper bound for the splitting) to be sharp, since for primary resonances the lower bounds (101) are given by the factors $\bar{b}_{n}$, which will can be arbitrarily close to $1-\delta$ for suitable $n$. Instead, in general the upper bound $J_{1}^{+}$(and hence the lower bound for the splitting) is far from being sharp, because it has been obtained in (102) by considering, for all $n$, the worst possible case in the bound $\bar{b}_{n} \leq 1+\delta$. In Sect. 3.3, we prove the sharpness of the lower bound $J_{0}^{-}$and show that, for a given frequency vector $\omega$, we can give (numerically) a sharp upper bound $J_{1}^{*}\left(\leq J_{1}^{+}\right)$, using the quasiperiodicity of the function $F_{1}(\zeta)$. In the same way, it would be enough to assume that $B_{0}^{-} \geq J_{1}^{*}$, instead of (100), in order to ensure that the splitting can be described in terms of only the primary resonances. This value $J_{1}^{*}$ is computed in Sect. 3.4 for the concrete case of the cubic golden frequency vector.

To end this section, we also deduce some useful properties of the function $S_{1}=S_{1}(\varepsilon)$, giving the dominant harmonic. Namely, this function is "piecewise-constant", with jump discontinuities exactly at the corners of $h_{1}(\varepsilon)$. Moreover, its asymptotic behavior as $\varepsilon \rightarrow 0$ turns out to be polynomial:

$$
\left|S_{1}(\varepsilon)\right| \sim \frac{1}{\varepsilon^{1 / 6}} .
$$

Indeed, the most dominant harmonic belongs to some resonant sequence: we can write $S_{1}(\varepsilon)=s(q, N)$ for some $q=q(\varepsilon)$, and for $N=N(\varepsilon)$ such that the value $\varepsilon_{s(q, N)}^{*}$ is close to $\varepsilon$, among the sequence $\varepsilon_{s(q, n)}^{*}, n \geq 0$. Recalling (77) and the estimate $|s(q, N)| \sim$ $\lambda^{N / 2}=\left(\lambda^{3 N}\right)^{1 / 6}$ deduced from (54), we get (103). Notice that it is not necessary to include $q$ in the estimate (103) (in spite of the fact that $K_{q}$ and $\tilde{\gamma}_{q}^{*}$ appear in the expression (77)), since only a finite number of resonant sequences $s(q, \cdot)$ is involved.

\subsection{Quasiperiodicity of the estimate of the most dominant harmonic. Now, our aim is} to show that the function $F_{1}(\zeta)$ is quasiperiodic with frequencies 1 and $\phi$. As we show below, this property is directly related to the oscillating factors $b_{s(q, n)}$ introduced in (56) for each resonant sequence, denoted $\bar{b}_{n}$ in (80) for the particular case of the primary resonances. Moreover, the facts that $\phi$ is an irrational number by Lemma 7, and $\delta>0$ by Lemma 9, allow us to ensure that the function $F_{1}(\zeta)$ is not periodic, which makes an important difference with respect to the case of quadratic frequencies considered in [DGG16].

Recall that, in (91), we wrote $F_{1}(\zeta)$ as the minimum of the functions $\widetilde{F}_{1}^{(q)}(\zeta)$, associated to each resonant sequence $s(q, n)$. Since all such functions are analogous to the function $\bar{F}_{1}(\zeta)$, associated to the primary resonances $s_{0}(n)$ and defined in (88), it is enough to show the quasiperiodicity of $\bar{F}_{1}(\zeta)$.

As a rough explanation for the frequencies 1 and $\phi$, notice that we can consider $\bar{F}_{1}(\zeta)$ as an $\mathcal{O}(\delta)$-perturbation of the function $\bar{F}_{1}^{(0)}(\zeta)$ introduced in (92), which is 1-periodic with respect to $\zeta$, and the oscillating factors $\bar{b}_{n}$ defined in (80) give rise to the second frequency $\phi$.

To be more precise, we are going to construct a positive, continuous and piecewisesmooth function $\Upsilon(x, y)$, defined on $\mathbb{R}^{2}$ and 1-periodic with respect to $x$ and $y$, such

\begin{tabular}{|l|l|l|l|l|l|}
\hline 220 & $\mathbf{3 8 3 2}$ & $\mathbf{B}$ & $\begin{array}{l}\text { Dispatch: 13/8/2020 } \\
\text { Total pages: 46 } \\
\text { Disk Received } \\
\text { Disk Used } \square\end{array}$ & $\begin{array}{l}\text { Journal: Commun. Math. Phys. } \\
\text { Not Used } \square \\
\text { Corrupted } \square \\
\text { Mismatch } \square\end{array}$ \\
\hline Jour. No & Ms. No.
\end{tabular}




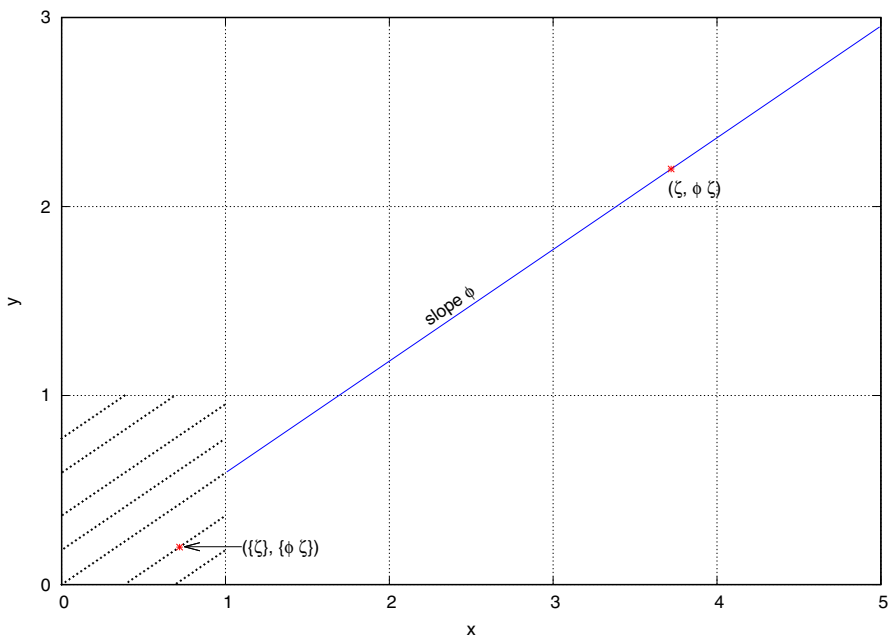

Fig. 3. The function $\Upsilon(x, y)$ on $\mathbb{R}^{2}$ "interpolating" $\bar{F}_{1}(\zeta)$ along the straight lines $x=\zeta, y=\phi \zeta$, and its reduction to the torus $\mathbb{T}_{*}^{2}$ (the slope $\phi \approx 0.590935$ corresponds to the case of the cubic golden vector)

that

$$
\Upsilon(\zeta, \phi \zeta)=\bar{F}_{1}(\zeta) \text { for any } \zeta \geq \zeta_{0}
$$

(for some $\zeta_{0}$ to be determined below, in Proposition 23). Equivalently, we can consider $\Upsilon(x, y)$ as defined on a torus $\mathbb{T}_{*}^{2}$, with $\mathbb{T}_{*}:=\mathbb{R} / \mathbb{Z}$ represented as the interval $[0,1)$, and the above equality can be rewritten as

$$
\begin{aligned}
& \Upsilon(\zeta,\{\phi(j+\zeta)\})=\bar{F}_{1}(j+\zeta) \\
& \quad \text { for any integer } j \geq 0 \text { and } \zeta \in[0,1) \text {, with } j+\zeta \geq \zeta_{0}
\end{aligned}
$$

where $\{a\} \in[0,1)$ denotes the fractional part of a given number $a \in \mathbb{R}$. This property of "interpolation" is illustrated in Fig. 3.

Like $\bar{F}_{1}(\zeta)$, defined in (88) as the minimum of the functions $\bar{f}_{n}(\zeta)$, the "interpolating" function $\Upsilon(x, y)$ will be defined in a similar way, as the minimum of a family functions. First of all, we define the 1-periodic function

$$
\beta(y):=1+\delta \cos \left(2 \pi \cdot y+2 \psi_{\widehat{q}}-\theta\right), \quad y \in \mathbb{R},
$$

and it is clear that the oscillating factors (80) are "interpolated" by this function: $\beta(\{n \phi\})=$ $\bar{b}_{n}$ for any $n$ (we can say that the values $\{n \phi\}$, filling densely the circle $\mathbb{T}_{*}$, are replaced by the continuous variable $y$ ). Now, recalling the "hyperbolic cosine-like" functions $\mathcal{C}(\zeta ; Z, Y)$ introduced in (82), we define for $n \in \mathbb{Z}$ the functions

$$
\chi_{n}(x, y):=\mathcal{C}(x ; n+\operatorname{Lg} \beta(y-\phi x+\{n \phi\}), \beta(y-\phi x+\{n \phi\})), \quad(x, y) \in \mathbb{R}^{2},
$$

which are clearly smooth and 1-periodic with respect to $y$, but not periodic with respect to $x$. Finally, we define

$$
\Upsilon(x, y):=\min _{n \in \mathbb{Z}} \chi_{n}(x, y)=\chi_{\widetilde{N}_{1}}(x, y), \quad(x, y) \in \mathbb{R}^{2},
$$

with $\widetilde{N}_{1}=\widetilde{N}_{1}(x, y)$ (compare with (88)).

\begin{tabular}{|l|l|l|l|l|l|}
\hline 220 & $\mathbf{3 8 3 2}$ & $\mathbf{B}$ & $\begin{array}{l}\text { Dispatch: 13/8/2020 } \\
\text { Total pages: 46 } \\
\text { Disk Received } \square \\
\text { Disk Used } \square\end{array}$ & $\begin{array}{l}\text { Journal: Commun. Math. Phys. } \\
\text { Not Used } \square \\
\text { Corrupted } \square \\
\text { Mismatch } \square\end{array}$ \\
\hline
\end{tabular}


It is clear that the functions $\chi_{n}(x, y)$ are closely related to the functions $\bar{f}_{n}(\zeta)$ defined in (85), as we see from the definition (106), by restricting $(x, y)$ to straight lines of slope $\phi$. To express this relationship more clearly we define, for any $y_{0} \in \mathbb{R}$, a function of one variable by restricting $\chi_{n}(x, y)$ to any straight line $y=y_{0}+\phi x$ for a given $y_{0}$,

$$
\begin{aligned}
\widehat{\chi}_{n}\left(x ; y_{0}\right) & :=\chi_{n}\left(x, y_{0}+\phi x\right)=\mathcal{C}\left(x ; \bar{x}_{n}\left(y_{0}\right), \bar{\beta}_{n}\left(y_{0}\right)\right), \\
\bar{x}_{n}\left(y_{0}\right) & :=n+\operatorname{Lg} \bar{\beta}_{n}\left(y_{0}\right), \quad \bar{\beta}_{n}\left(y_{0}\right):=\beta\left(y_{0}+\{n \phi\}\right)
\end{aligned}
$$

(compare with (85)). We can also define

$$
\widehat{\Upsilon}\left(x ; y_{0}\right):=\min _{n \in \mathbb{Z}} \widehat{\chi}_{n}\left(x ; y_{0}\right)=\widehat{\chi}_{\widehat{N}_{1}}\left(x ; y_{0}\right),
$$

and it is clear that $\widehat{\Upsilon}\left(x ; y_{0}\right)=\Upsilon\left(x, y_{0}+\phi x\right)$, and also $\widehat{N}_{1}\left(x ; y_{0}\right)=\widetilde{N}_{1}\left(x, y_{0}+\phi x\right)$ (with the difference that $\Upsilon$ is 1-periodic and can be reduced to $\mathbb{T}_{*}^{2}$, see Proposition 23, but the periodicity with respect to $x$ does not hold for $\widehat{\Upsilon}$ ).

Some of the properties stated in the following lemma are clearly inherited from the results of Lemmas 16, 17 and 19.

Lemma 22. (a) The functions $\chi_{n}(x, y)$ are smooth and 1-periodic with respect to $y$, and satisfy the following translation property:

$$
\chi_{n}(x+1, y)=\chi_{n-1}(x, y), \text { for any }(x, y) \in \mathbb{R}^{2}, \quad n \in \mathbb{Z} .
$$

(b) For any given $n$ and $y_{0} \in \mathbb{R}$, the function $\widehat{\chi}_{n}\left(x ; y_{0}\right)$ is convex (with respect to $x$ ) and attains its minimum at $x=\bar{x}_{n}\left(y_{0}\right)$, with the minimum value $\bar{\beta}_{n}\left(y_{0}\right)^{1 / 3}$. The dependence of $\widehat{\chi}_{n}\left(x ; y_{0}\right)$ on the parameter $y_{0}$ is 1-periodic.

(c) For any given $n$, the function $\chi_{n}(x, y)$ attains its minimum at the point $(x, y)=$ $\left(\tilde{x}_{n}, \tilde{y}_{n}\right)$, with

$$
\tilde{x}_{n}=n+\operatorname{Lg}(1-\delta), \quad \tilde{y}_{n} \equiv \frac{\pi-2 \psi \widehat{q}+\theta}{2 \pi}+\phi \operatorname{Lg}(1-\delta) \quad(\bmod 1),
$$

with the minimum value $(1-\delta)^{1 / 3}$.

(d) For any given $n, m$ with $n \neq m$, and $y_{0} \in \mathbb{R}$, the functions $\widehat{\chi}_{n}\left(x ; y_{0}\right)$ and $\widehat{\chi}_{m}\left(x ; y_{0}\right)$ do not coincide. Their graphs intersect transversely at a unique point, or do not intersect. The set $\mathcal{Y}_{n, m}$ of values $y_{0}$ such that the intersection exists is a union of open intervals (or eventually $\mathcal{Y}_{n, m}=\mathbb{R}, \mathcal{Y}_{n, m}=\emptyset$ ). For $y_{0} \in \mathcal{Y}_{n, m}$, the intersecting point $x=x_{n, m}^{*}\left(y_{0}\right)$ (given explicitly in (110)) is a smooth and 1-periodic function of $y_{0}$.

(e) For any given $n, m$ with $n \neq m$, the graphs of the functions $\chi_{n}(x, y)$ and $\chi_{m}(x, y)$ intersect (if they do) transversely along the curves parameterized by

$$
x=x_{n, m}^{*}\left(y_{0}\right), \quad y=y_{0}+\phi x_{n, m}^{*}\left(y_{0}\right), \quad y_{0} \in \mathcal{Y}_{n, m} .
$$

(f) For any $(x, y)$, we have $N_{1}^{(0)}(x)-N^{-} \leq \tilde{N}_{1}(x, y) \leq N_{1}^{(0)}(x)+N^{+}$, with $N_{1}^{(0)}(x)$ as in (92), and $N^{ \pm}=N^{ \pm}(\omega)$ as in Lemma 19 .

Proof. The only assertion to be checked in (a) is the translation property. For that, it is enough to ensure that

$$
\beta(y-\phi(x+1)+\{n \phi\})=\beta(y-\phi x+\{(n-1) \phi\}),
$$

but this is a direct consequence of the 1-periodicity of $\beta(y)$. The proof of (b) is straightforward from the definition of the functions $\widehat{\chi}_{n}\left(x ; y_{0}\right)$ in (108). We also get (c) as a

$\approx$


direct consequence of (b), choosing $y_{0}=y_{0}^{(n)}$ such that $\bar{\beta}_{n}\left(y_{0}\right)$ attains its minimum value $1-\delta$, and hence $\tilde{x}_{n}=\bar{x}_{n}\left(y_{0}^{(n)}\right), \tilde{y}_{n}=y_{0}^{(n)}+\phi \tilde{x}_{n}$.

For $(\mathrm{d})$, we first notice that the functions $\widehat{\chi}_{n}\left(x ; y_{0}\right)$ and $\widehat{\chi}_{m}\left(x ; y_{0}\right)$ do not coincide, since $\bar{\beta}_{n}\left(y_{0}\right) \neq \bar{\beta}_{m}\left(y_{0}\right)$ (due to the irrationality of $\phi$ ). Then, we directly apply Lemma 17 with $Z=\bar{x}_{m}\left(y_{0}\right)-\bar{x}_{n}\left(y_{0}\right)$ and $W=\left(\bar{\beta}_{m}\left(y_{0}\right) / \bar{\beta}_{n}\left(y_{0}\right)\right)^{1 / 3}$. We get the formula for the intersecting point,

$$
x_{n, m}^{*}\left(y_{0}\right)=\bar{x}_{n}\left(y_{0}\right)+2 \operatorname{Lg} \frac{2 \lambda^{Z}\left(W \lambda^{Z / 2}-1\right)}{\lambda^{Z}-W} .
$$

If the intersection exists, it is unique, but its existence may depend on $y_{0}$, according to the condition given in Lemma 17. We also get (e) as a direct consequence of (d).

Finally, for the proof of (f), for any $y_{0}$ we consider the function $\widehat{\Upsilon}\left(x ; y_{0}\right)$ defined in (109), and it is enough to prove that $N_{1}^{(0)}(x)-N^{-} \leq \widehat{N}_{1}\left(x ; y_{0}\right) \leq N_{1}^{(0)}(x)+N^{+}$. Now, we can use that the functions $\widehat{\chi}_{n}\left(x ; y_{0}\right)$ introduced in $(108)$ are completely analogous to the functions $\bar{f}_{n}(\zeta)$ in (85), replacing $\bar{b}_{n}$ by $\bar{\beta}_{n}\left(y_{0}\right)$, and $\bar{\zeta}_{n}$ by $\bar{x}_{n}\left(y_{0}\right)$. Then, the proof follows exactly as in Lemma 19 , using the values of $Z$ and $W$ defined above.

Proposition 23. The function $\Upsilon(x, y)$ is continuous and piecewise-smooth, and 1 -periodic with respect to $x$ and $y$, and satisfies the "interpolation" property (104) for $\zeta \geq \zeta_{0}:=$ $N^{-}+\xi_{0}$ (recall that $\xi_{0}$ is defined in (93)).

Proof. First of all, from definitions (85) and (106), it is not hard to see that the equality $\chi_{n}(\zeta, \phi \zeta)=\widehat{\chi}_{n}(\zeta ; 0)=\bar{f}_{n}(\zeta)$ is fulfilled for any $n \geq 0$ and $\zeta \in \mathbb{R}$ (we only have to use that $\left.\bar{\beta}_{n}(0)=\bar{b}_{n}\right)$. By Lemma 22(f), we can take the minimum over $n$ by restricting ourselves to a finite number of cases, $N_{1}^{(0)}(\zeta)-N^{-} \leq n \leq N_{1}^{(0)}(\zeta)+N^{+}$, and we directly get the equality (104), or equivalently (105). However, in order to ensure that $n \geq 0$ as in the definition (88), we need that $N_{1}^{(0)}(\zeta) \geq N^{-}$. As can be seen in (92), we have $N_{1}^{(0)}(\zeta) \geq \zeta-\xi_{0}$, and hence we assume $\zeta \geq N^{-}+\xi_{0}$.

The fact that $\Upsilon(x, y)$ is, for any $(x, y)$, the minimum of a finite number of smooth functions ensures that it is continuous and piecewise-smooth. It is also clear that it is periodic with respect to $y$, since so are the functions $\chi_{n}(x, y)$. Finally, its periodicity with respect to $x$ is easily deduced from the translation property of Lemma 22(a).

In this way, by studying the function $\Upsilon(x, y)$ on the torus $\mathbb{T}_{*}^{2}$ we can determine the intervals of dominance for the function $\bar{F}_{1}(\zeta)$, in (88). It is enough to divide $\mathbb{T}_{*}^{2}$ into a finite number of regions, according to the function $\chi_{n}(x, y)$ giving the minimum in (107). Since for $x \in[0,1)$ the index $N_{1}^{(0)}(x)$ is either 0 or 1, by Lemma 22(f) it is enough to consider the functions $\chi_{n}(x, y)$ with $-N^{-} \leq n \leq 1+N^{+}$. The regions visited by the straight line $(\zeta, \phi \zeta)$ correspond the intervals of dominance for $\bar{F}_{1}(\zeta)$. See Fig. 4 for an illustration, for the concrete case of the cubic golden vector (we point out that the borders between neighbor regions are not straight lines, but rather pieces of the curves parameterized in Lemma 22(e)).

Numerically, we can obtain sharp bounds for the function $\bar{F}_{1}(\zeta)$, improving the ones given in Proposition 20. Since $\phi$ is irrational, the line $(\zeta, \phi \zeta)$ fills densely the torus $\mathbb{T}_{*}^{2}$ and hence

$$
\inf \bar{F}_{1}(\zeta)=\min \Upsilon(x, y)=J_{0}^{-}, \quad \sup \bar{F}_{1}(\zeta)=\max \Upsilon(x, y) \leq J_{1}^{+} .
$$

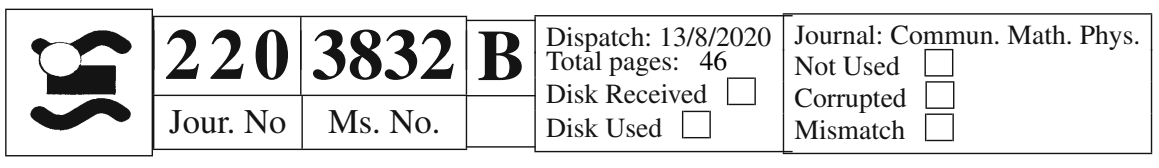



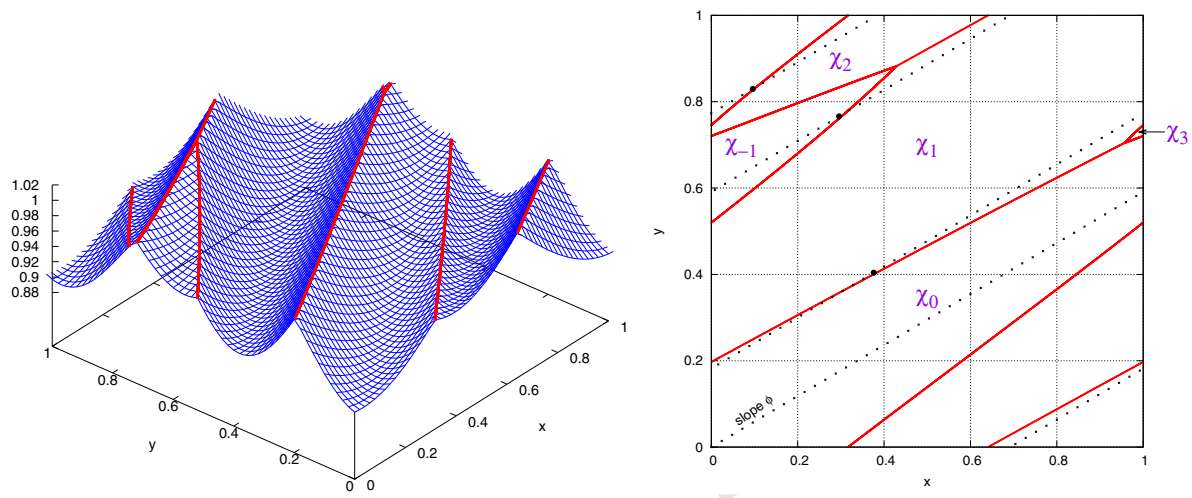

Fig. 4. Graph of the function $\Upsilon(x, y)$ on its domain $\mathbb{T}_{*}^{2}$, as the minimum of the functions $\chi_{n}(x, y)$, for the cubic golden vector. The red curves (which are not straight lines) are the borders between the regions of dominance, where a different function $\chi_{n}(x, y)$ gives the minimum in (107). The function $\bar{F}_{1}(\zeta)$ is the restriction of $\Upsilon(x, y)$ along the dotted line of slope $\phi$, by the property of "interpolation", see (104-105). The changes in the dominance, which take place when the line of slope $\phi$ crosses a red curve, correspond to the corners of $\bar{F}_{1}(\zeta)$ in Fig. 1

The minimum value $J_{0}^{-}=(1-\delta)^{1 / 3}$ of $\Upsilon(x, y)$ is attained at the point given in Lemma 22(c), choosing $n$ such that $\tilde{x}_{n} \in[0,1)$. On the other hand, by the convexity of $\Upsilon$ along the lines of slope $\phi$, the maximum value

$$
J_{1}^{*}:=\max \Upsilon(x, y)
$$

is attained at some point belonging to some of the curves limiting the regions of dominance illustrated in Fig. 4, Recall that the values $J_{0}^{-}$and $J_{1}^{*}$ are associated, respectively, to sharp upper and lower bounds for the maximum splitting distance (see Remark 2(a)). Again, see Sect. 3.4 for the case of the cubic golden vector.

3.4. The particular case of the cubic golden frequency vector. As a continuation of Sect. 2.3, we provide particular data concerning the function $h_{1}(\varepsilon)=F_{1}(\zeta)$, and hence the asymptotic estimate for the splitting, for the concrete case of the cubic golden frequency vector introduced in (67).

First of all, recall that the function $\bar{F}_{1}(\zeta)$ defined in (88), associated to the primary resonances, is an $\mathcal{O}(\delta)$-perturbation of the 1-periodic function $\bar{F}_{1}^{(0)}(\zeta)$ introduced in (92). This one reaches its minimum value at the points $\zeta_{n}=n$, and its maximum value at the points $\zeta_{n}^{\prime}=n+\xi_{0}$, with $\xi_{0} \approx 0.492049$ in (93), where we have used the value of $\lambda$ obtained in (68). The minimum value is 1 and the maximum value is $J_{1}^{(0)} \approx 1.009141$ by (95).

For the "perturbed" function $\bar{F}_{1}(\zeta)$, we use the value of $\delta$ obtained in (69) and, in Lemma 19 , we get the values $N^{-} \approx 3.65$ and $N^{+} \approx 3.97$. This says that, for $\zeta$ belonging to a given interval $\mathcal{I}_{n}=\left[\zeta_{n-1}^{\prime}, \zeta_{n}^{\prime}\right]\left(\right.$ where we have $\left.N_{1}^{(0)}(\zeta)=n\right)$, we can compute $\bar{F}_{1}(\zeta)$ as the minimum of the functions $\bar{f}_{j}(\zeta)$ for $n-3 \leq j \leq n+3$.

On the other hand, by Proposition 20 we have the following lower and upper bounds for $\bar{F}_{1}(\zeta)$

$$
J_{0}^{-} \approx 0.892341, \quad J_{1}^{+} \approx 1.098383 .
$$

二


The strong separation condition (100) is fulfilled for the cubic golden vector, since the value $B_{0}^{-}$obtained in (70) is clearly greater than $J_{1}^{+}$, and hence $\bar{F}_{1}(\zeta)=F_{1}(\zeta)$ for this example. In fact, the upper bound $J_{1}^{+}$can be replaced by the sharp upper bound $J_{1}^{*}$ defined in (111), and numerically we see that

$$
J_{1}^{*} \approx 1.010619
$$

(this value is reached at the confluence of the regions where $\chi_{-1}, \chi_{1}, \chi_{2}$ are dominant, see Fig. 4).

\section{Justification of the Asymptotic Estimate}

We consider in this section the final step in the proof of our main result (Theorem 1), which gives an exponentially small asymptotic estimate for the maximal splitting distance, i.e. the maximum of $|\mathcal{M}(\theta)|$. We write the Poincaré-Melnikov approximation (3) as

$$
\mathcal{M}(\theta)=\mu M(\theta)+\mathcal{R}(\theta)
$$

where $\mathcal{R}(\theta)$ denotes the remainder. Our aim is to ensure that the Poincaré-Melnikov method predicts correctly the size of the splitting in the singular case $\mu=\varepsilon^{r}$, extending our results in the previous section for the Melnikov function $M(\theta)$, to the whole splitting function $\mathcal{M}(\theta)$. Recalling that such functions are gradients of scalar functions (see (2) and (16)), it will be enough to work with the Melnikov and splitting potentials $L(\theta)$ and $\mathcal{L}(\theta)$, which appear below in Lemma 24 . Our approach requires the following steps:

1. An asymptotic estimate for the dominant harmonic, given by $k=S_{1}(\varepsilon)$, of the Melnikov potential $L(\theta)$;

2. An upper bound for the harmonics of the error term $\mathcal{R}(\theta)$ in (112), mainly the one associated to $k=S_{1}(\varepsilon)$, showing that it is also dominated by the asymptotic estimate of the dominant harmonic of the first order approximation;

3. An upper bound for the sum of the non-dominant terms of the Fourier expansion of the splitting potential $\mathcal{L}(\theta)$, ensuring that it can be approximated by its dominant harmonic.

In other words, we need to show that the asymptotic estimate for the dominant harmonic in the Poincaré-Melnikov approximation is large enough to overcome the corresponding harmonic of the error term, as well as an upper bound of its remaining harmonics.

The first step in the above list has been carried out in the previous section, and it is the only step that depends strongly on the arithmetic properties of the frequency vector. In this section, we outline the second and third steps, which are analogous to the case of the quadratic golden number done in [DG04] (see also [DGG16]), and do not require to use the specific arithmetic properties of cubic frequency vectors. The upper bounds required in such steps are given in [DGS04], and are valid for any dimension of the frequency vector $\omega$, assuming only that it satisfies a Diophantine condition.

We start with describing our approach in a few words. First of all, notice that Theorem 1 is stated in terms of the splitting function $\mathcal{M}=\nabla \mathcal{L}$ introduced in (15). We write, for the splitting potential and function,

$$
\mathcal{L}(\theta)=\sum_{k \in \mathcal{Z} \backslash\{0\}} \mathcal{L}_{k} \cos \left(\langle k, \theta\rangle-\tau_{k}\right), \quad \mathcal{M}(\theta)=-\sum_{k \in \mathcal{Z} \backslash\{0\}} \mathcal{M}_{k} \sin \left(\langle k, \theta\rangle-\tau_{k}\right)(113)
$$

\begin{tabular}{|l|l|l|l|l|l|}
\hline 220 & $\mathbf{3 8 3 2}$ & $\mathbf{B}$ & $\begin{array}{l}\text { Dispatch: 13/8/2020 } \\
\text { Total pages: 46 } \\
\text { Disk Received } \\
\text { Disk Used } \square\end{array}$ & $\begin{array}{l}\text { Journal: Commun. Math. Phys. } \\
\text { Not Used } \square \\
\text { Corrupted } \square \\
\text { Mismatch } \square\end{array}$ \\
\hline
\end{tabular}


with scalar (positive) coefficients $\mathcal{L}_{k}$, and vector coefficients

$$
\mathcal{M}_{k}=k \mathcal{L}_{k} \in \mathbb{R}^{3} .
$$

Although the Melnikov approximation (112) is in principle valid for real $\theta$, it is standard to see that it can be extended to a complex strip of suitable width (see for instance [DGS04]), from which one gets upper bounds for $\left|\mathcal{L}_{k}-\mu L_{k}\right|$, which imply the estimates given below in Lemma 24, ensuring that the most dominant harmonic of the Melnikov potential $L(\theta)$, obtained for $k=S_{1}(\varepsilon)$ (see (87)), is also the dominant one for the splitting potential $\mathcal{L}(\theta)$. Then, this dominant harmonic determines the asymptotic estimate for the maximal splitting distance, given in Theorem 1.

With this idea, we consider the approximation of $\mathcal{L}(\theta)$ given by its dominant harmonic, as well as the corresponding remainder,

$$
\begin{aligned}
\mathcal{L}(\theta) & =\mathcal{L}^{(1)}(\theta)+\mathcal{F}^{(2)}(\theta), \\
\mathcal{L}^{(1)}(\theta) & :=\mathcal{L}_{S_{1}} \cos \left(\left\langle S_{1}, \theta\right\rangle-\tau_{S_{1}}\right), \quad \mathcal{F}^{(2)}(\theta):=\sum_{k \in \mathcal{Z}_{2}} \mathcal{L}_{k} \cos \left(\langle k, \theta\rangle-\tau_{k}\right),
\end{aligned}
$$

where we denote $\mathcal{Z}_{2}:=\mathcal{Z} \backslash\left\{0, S_{1}\right\}$, and we give below, in Lemma 24 , an estimate for the sum of all harmonics in the remainder $\mathcal{F}^{(2)}(\theta)$, in order to ensure that the maximal splitting distance can be approximated by the size of the coefficient of the most dominant harmonic $S_{1}(\varepsilon)$. In fact, the estimate for $\mathcal{F}^{(2)}(\theta)$ is also given, by the exponential smallness of the harmonics, in terms of its own dominant harmonic in the set $\mathcal{Z}_{2}$, that we denote as $S_{2}(\varepsilon)$. With this in mind, we introduce as in (87) the continuous and piecewise-smooth function

$$
h_{2}(\varepsilon):=\min _{k \in \mathcal{A} \backslash\left\{S_{1}\right\}} g_{k}^{*}(\varepsilon)=g_{S_{2}}^{*}(\varepsilon) .
$$

It is not hard to see from Lemmas 16 and 17 that the corners of $h_{1}(\varepsilon)$, at which a change in the first dominant harmonic takes place, are exactly the points $\check{\varepsilon}$ such that $h_{1}(\breve{\varepsilon})=h_{2}(\breve{\varepsilon})$ (such points are also the "lower corners" of $h_{2}(\varepsilon)$, but this function also has "upper corners" where it coincides with the analogous function $h_{3}(\varepsilon)$ associated to the third dominant harmonic; see [DGG16]).

The following lemma, analogous to the one established in [DG03,DG04], provides an asymptotic estimate for the dominant harmonic $\mathcal{L}_{S_{1}}$, as well as an estimate for the sum of all the harmonics in the remainder appearing in (115), As said before, we are not directly interested in the splitting potential $\mathcal{L}(\theta)$, but rather its derivative $\mathcal{M}(\theta)$. Recall that the coefficients $\mathcal{L}_{k}$, introduced in (113), are all positive, and that the constant $C_{0}$ in the exponentials has been defined in (74). On the other hand, we use the following notation: for positive quantities, we write $f \preceq g$ if we can bound $f \leq c g$ with some (positive) constant $c$ not depending on $\varepsilon$ and $\mu$. In this way, we can write $f \sim g$ if $g \preceq f \preceq g$, as already defined just before the statement of Theorem 1 .

Lemma 24. For $\varepsilon$ small enough and $\mu=\varepsilon^{r}$ with $r>3$, one has:

(a) $\mathcal{L}_{S_{1}} \sim \mu L_{S_{1}} \sim \frac{\mu}{\varepsilon^{1 / 6}} \exp \left\{-\frac{C_{0} h_{1}(\varepsilon)}{\varepsilon^{1 / 6}}\right\}$;

(b) $\sum_{k \in \mathcal{Z}_{2}} \mathcal{L}_{k} \sim \frac{1}{\varepsilon^{1 / 3}} \mathcal{L}_{S_{2}} \sim \frac{\mu}{\varepsilon^{1 / 3}} \exp \left\{-\frac{C_{0} h_{2}(\varepsilon)}{\varepsilon^{1 / 6}}\right\}$. 
Sketch of the proof. We only give the main ideas of the proof, since it is similar to analogous results in [DG04, Lemmas 4 and 5] and [DG03, Lemma 3]. Our aim is to show that, at first order in $\mu$, the coefficients of the splitting potential can be approximated by the coefficients of the Melnikov potential, i.e. the coefficients $\mathcal{R}_{k}$ of the error term in the Melnikov approximation (112) can be neglected: $\left|\mathcal{R}_{k}\right| \ll \mu|k|\left|L_{k}\right|$, and hence

$$
\mathcal{L}_{k} \sim \mu L_{k}=\mu \alpha_{k} \mathrm{e}^{-\beta_{k}},
$$

with the exponents $\beta_{k}=\beta_{k}(\varepsilon)$ and the factors $\alpha_{k}=\alpha_{k}(\varepsilon)$ introduced in (72-73).

As said at the beginning of this section, the estimates for the error term come from upper bounds given in the paper [DGS04], where a quite general setting is considered. The application of such upper bounds to our case is completely analogous to the case of the golden quadratic frequencies considered in [DG04], differing only in some involved exponents in (118-119) and (123).

To start, we see that the hypotheses in [DGS04, p. 788] are satisfied in our case, and allow us to introduce the following values $n, \tau, l$ and $\alpha$ :

* the frequency vector $\omega$ has dimension $n=3$ and satisfies the Diophantine condition (7) with the exponent $\tau=2$;

* the function $h(x)$ in (9) is a trigonometric polynomial of degree $l=1$, and hence $h\left(x_{0}(s)\right)$ (see (11)) has poles of order $2 l=2$ at $s= \pm \mathbf{i} \pi / 2$;

* the function $f(\varphi)$ in (9) is analytic in a complex strip $|\operatorname{Im} \varphi|<\rho$ and, for any $0<\delta<\rho$, satisfies a bound $\|f\|_{\rho-\delta} \preceq 1 / \delta^{\alpha}$ with $\alpha=3$ (where $\|f\|_{\rho-\delta}$ denotes a norm on the strip $|\operatorname{Im} \varphi| \leq \rho-\delta$ taking into account the Fourier expansion of $f(\varphi)$, see [DGS04, p. 791] for a precise definition). This hypothesis provides a control on the size of the perturbation near a "pole-like singularity of order $\alpha$ ".

In this situation, we know from [DGS04, Th. 10] that the "full" splitting function introduced in (13) is a gradient in the angular variables, $\widetilde{\mathcal{M}}(s, \theta)=\partial_{\theta} \widetilde{\mathcal{L}}(s, \theta)$, and it is $\omega_{\varepsilon}$-quasiperiodic (see (14)) and analytic on a complex strip

$$
|s| \leq \kappa-\delta, \quad|\operatorname{Im} s| \leq \frac{\pi}{2}-\delta, \quad \operatorname{Re} \theta \in \mathbb{T}^{3}, \quad|\operatorname{Im} \varphi| \leq \rho-\delta
$$

for any given small $\delta=\delta(\varepsilon)$ (to be chosen below appropriately), with an upper bound for the remainder in such a strip. To write this upper bound, we denote $\mathcal{R}(s, \theta):=$ $\widetilde{\mathcal{M}}(s, \theta)-\mu M\left(\theta-\omega_{\varepsilon} s\right)$ the "full" remainder, and its supremum norm in the strip (117) satisfies a bound of the type

$$
|\widetilde{\mathcal{R}}|_{\kappa-\delta, \frac{\pi}{2}-\delta, \rho-\delta} \preceq \frac{\mu^{2}}{\delta^{20}}+\frac{\mu^{2}}{\delta^{17} \sqrt{\varepsilon}},
$$

provided we assume

$$
\varepsilon \preceq 1, \quad \mu \preceq \delta^{12}, \quad \mu \preceq \delta^{7} \sqrt{\varepsilon} .
$$

The exponents of $\delta$ in (118-119) have been computed through the formulas in [DGS04, pp. 792-793], from the values $n=3, \tau=2, l=1$ and $\alpha=3$.

The $\omega_{\varepsilon}$-quasiperiodicity plays an essential role, since it implies that the remainder $\mathcal{R}(\theta)=\mathcal{R}(0, \theta)$ is exponentially small in $\varepsilon$ on the real domain, $\theta \in \mathbb{T}^{3}$. Notice that, since the Melnikov and splitting functions $M$ and $\mathcal{M}$ are both gradients, the remainder

二


$\mathcal{R}=\mathcal{M}-\mu M$ is also a gradient, and hence it has zero average: $\mathcal{R}_{0}=0$. For its remaining Fourier coefficients $\mathcal{R}_{k}, k \neq 0$, we get from [DGS04, Lemma 11] the following bound,

$$
\left|\mathcal{R}_{k}\right| \leq|\widetilde{\mathcal{R}}|_{\kappa-\delta, \frac{\pi}{2}-\delta, \rho-\delta} \mathrm{e}^{-\widehat{\beta}_{k}(\varepsilon, \delta)} \cdot \widehat{\beta}_{k}(\varepsilon, \delta):=(\rho-\delta)|k|+\left(\frac{\pi}{2}-\delta\right)\left|\left\langle k, \omega_{\varepsilon}\right\rangle\right| .
$$

Notice that the new exponents $\widehat{\beta}_{k}(\varepsilon, \delta)$ are somewhat smaller than the exponents $\beta_{k}(\varepsilon)$ for the coefficients of the Melnikov potential, introduced in (73).

As mentioned in Sect. 3.1, the main behavior of the coefficients $L_{k}(\varepsilon)$ is given by the exponents $\beta_{k}(\varepsilon)$, which have been written in (74) in terms of the functions $g_{k}(\varepsilon)$. We focus our attention on the coefficient $L_{S_{1}}$, associated to the dominant harmonic $k=S_{1}(\varepsilon)$, which can be expressed in terms of the function $h_{1}(\varepsilon)$ introduced in (87). In this way, we obtain an estimate for the factor $\mathrm{e}^{-\beta_{S_{1}}}$, which provides the exponential factor in (a). We also consider the factor $\alpha_{k}$, with $k=S_{1}(\varepsilon)$. Recalling from (103) that $\left|S_{1}\right| \sim \varepsilon^{-1 / 6}$, we get from (72) that $\alpha_{S_{1}} \sim \varepsilon^{-1 / 6}$, which provides the polynomial factor in part (a). We also get an exponential estimate for the dominant term of the Melnikov funcion,

$$
\left|M_{S_{1}}\right|=\left|S_{1}\right| L_{S_{1}} \sim \frac{\mu}{\varepsilon^{1 / 3}} \exp \left\{-\frac{C_{0} h_{1}(\varepsilon)}{\varepsilon^{1 / 6}}\right\} .
$$

The estimate obtained is valid for the dominant coefficient $L_{S_{1}}$ of the Melnikov potential $L(\theta)$. To complete the proof of part (a), one has to show that an analogous estimate is also valid for the coefficient $\mathcal{L}_{S_{1}}$ of the splitting potential $\mathcal{L}(\theta)$, i.e. when the error term $\mathcal{R}(\theta)$ in the Poincaré-Melnikov approximation (112) is not neglected. Notice that $\left|\mathcal{M}_{S_{1}}\right|=\left|S_{1}\right| \mathcal{L}_{S_{1}}$ and $\mu\left|M_{S_{1}}\right|=\mu\left|S_{1}\right| L_{S_{1}}$ are, respectively, the maximum value of the harmonics $\mathcal{M}_{S_{1}} \sin \left(\left\langle S_{1}, \theta\right\rangle-\tau_{S_{1}}\right)$ and $\mu M_{S_{1}} \sin \left(\left\langle S_{1}, \theta\right\rangle-\sigma_{S_{1}}\right)$ (see (113)), and their difference is the corresponding harmonic of $\mathcal{R}(\theta)$, whose maximum value is $\left|\mathcal{R}_{S_{1}}\right|$. Then, we have the bound $\left|S_{1}\right|\left|\mathcal{L}_{S_{1}}-\mu L_{S_{1}}\right| \leq\left|\mathcal{R}_{S_{1}}\right|$, and we have to show that, in our singular case $\mu=\varepsilon^{r}$, the coefficient of the error term is dominated by the one for the Melnikov function:

$$
\left|\mathcal{R}_{S_{1}}\right| \ll \mu\left|S_{1}\right| L_{S_{1}} .
$$

Since this can be worked out straightforwardly as in [DG04, Lemma 5], we give here only the main ideas. We know from the upper bound (120) that $\left|\mathcal{R}_{S_{1}}\right|$ is also exponentially small, but the main difficulty lies in the fact that the exponential factor $\mathrm{e}^{-\widehat{\beta}_{S_{1}}}$ in (120) is somewhat greater than the exponential factor $\mathrm{e}^{-\beta_{S_{1}}}$ in (121) (as we see by comparing the expressions of the exponents $\beta_{S_{1}}$ and $\widehat{\beta}_{S_{1}}$ ). This difficulty can be solved with an appropiate choice of $\delta$. Indeed, when such exponents are expressed in terms of the function $h_{1}(\varepsilon)$, we see that the numerator $C_{0}$ is replaced by another numerator $\widetilde{C}_{0}(\delta)=$ $C_{0}+\mathcal{O}(\delta)$ obtained by replacing $\rho$ and $\pi / 2$ by $\rho-\delta$ and $\pi / 2-\delta$, respectively, in the definition (74). Choosing

$$
\delta=\varepsilon^{1 / 6},
$$

it turns out that both exponents are of the same order, since $C_{0} \varepsilon^{-1 / 6} \sim \widetilde{C}_{0}(\delta) \varepsilon^{-1 / 6}$. Once this equivalence has been established, we only have to compare the polynomial factors i.e. we need that $\frac{\mu^{2}}{\delta^{20}}+\frac{\mu^{2}}{\delta^{17} \sqrt{\varepsilon}}=\frac{2 \mu^{2}}{\varepsilon^{10 / 3}} \ll \frac{\mu}{\varepsilon^{1 / 3}}$, which is true for $\mu=\varepsilon^{r}$ if 
$r>3$. The assumptions (119) are also satisfied with this choice of $r$, and this proves the dominance (122).

The proof of part (b) is carried out in similar terms. For the dominant harmonic $k=S_{2}(\varepsilon)$ inside the set $\mathcal{Z}_{2}$, we also get $\left|S_{2}\right| \sim \varepsilon^{-1 / 6}$ as in (103), and an exponentially small estimate for $\mathcal{L}_{S_{2}}$ with the function $h_{2}(\varepsilon)$ defined in (116). Such estimates are also valid if one considers the whole sum in (b), since for any given $\varepsilon$ the terms of this sum can be bounded by a geometric series and, hence, it can be estimated by its dominant term (see [DG04, Lemma 4] for more details).

With regard to the proof of Theorem 1, we need to measure the size of the perturbation $\mathcal{F}^{(2)}(\theta)$ in $(115)$ with respect to the coefficient $\mathcal{L}_{S_{1}}$ of the approximation $\mathcal{L}^{(1)}(\theta)$. Since by Lemma 24 the size of $\mathcal{F}^{(2)}(\theta)$ is given by the size of its dominant harmonic, we introduce the following small parameter,

$$
\eta_{2,1}:=\frac{\mathcal{L}_{S_{2}}}{\mathcal{L}_{S_{1}}} \sim \exp \left\{-\frac{C_{0}\left(h_{2}(\varepsilon)-h_{1}(\varepsilon)\right)}{\varepsilon^{1 / 6}}\right\},
$$

as a measure of the perturbation $\mathcal{F}^{(2)}(\theta)$ in (115), relatively to the size of the dominant coefficient $\mathcal{L}_{S_{1}}$. Although we define the parameter $\eta_{2,1}$ in terms of the coefficients of $\mathcal{L}(\theta)$, we can also define it from the coefficients of its derivative, the splitting function $\mathcal{M}(\theta)=\nabla \mathcal{L}(\theta)$, in view of (114) and the fact that the respective factors have the same magnitude: $\left|S_{1}\right| \sim\left|S_{2}\right| \sim \varepsilon^{-1 / 6}$.

Notice that the parameter $\eta_{2,1}$ is always exponentially small in $\varepsilon$, provided we exclude some small neighborhoods of the "transition values" $\check{\varepsilon}$, where $\mathcal{L}_{S_{1}}$ and $\mathcal{L}_{S_{2}}$ have the same magnitude.

Proof of Theorem 1. Applying Lemma 24, we see that the coefficient of the dominant harmonic of the splitting function $\mathcal{M}(\theta)$ is greater than the sum of all other harmonics. More precisely, we have for $\varepsilon \rightarrow 0$ the estimate

$$
\max _{\theta \in \mathbb{T}^{3}}|\mathcal{M}(\theta)|=\left|\mathcal{M}_{S_{1}}\right|\left(1+\mathcal{O}\left(\eta_{2,1}\right)\right) \sim\left|\mathcal{M}_{S_{1}}\right| \sim\left|S_{1}\right| \mathcal{L}_{S_{1}},
$$

which implies the result, using the asymptotic estimate (103) for $\left|S_{1}\right|$, and the asymptotic estimate for $\left|\mathcal{M}_{S_{1}}\right|$, in terms of $h_{1}(\varepsilon)$, deduced from Lemma 24(a).

Nevertheless, the previous argument does not apply directly when $\varepsilon$ is close to a transition value $\check{\varepsilon}$ where $h_{1}$ and $h_{2}$ coincide, i.e. the first and second dominant harmonics have the same magnitude. Eventually, more than two harmonics (but a finite number, according to the arguments given in Lemma 17) might also have the same magnitude and become dominant. In such cases, the parameter $\eta_{2,1}$ is not exponentially small, but we can replace the main term in (124) by a finite number of terms, plus an exponentially small perturbation, and by the properties of Fourier expansions the maximum value of $|\mathcal{M}(\theta)|$ can be compared to any of its dominant harmonics.

Acknowledgments. We would like to express our sincere thanks to Carles Simó for useful discussions and remarks on resonances and Diophantine vectors, and to Bernat Plans for some useful hints on algebraic number theory. We also acknowledge the use of EIXAM, the UPC Applied Math cluster system for research computing (https://dynamicalsystems.upc.edu/en/computing), and in particular Albert Granados for his support in the use of this cluster. The author MG also thanks the Dep. de Matemàtiques i Informàtica of the Univ. de Barcelona for their hospitality and support. We also thank the referees, whose remarks have helped us to improve the structure and clearness of this paper.

Publisher's Note Springer Nature remains neutral with regard to jurisdictional claims in published maps and institutional affiliations.

$\approx$




\section{References}

[Arn64] Arnold, V.I.: Instability of dynamical systems with several degrees of freedom. Sov. Math. Dokl. 5(3), 581-585 (1964). (Dokl. Akad. Nauk SSSR 156, 9-12 (1964))

[Bal06] Baldomá, I.: The inner equation for one and a half degrees of freedom rapidly forced Hamiltonian systems. Nonlinearity 19(6), 1415-1445 (2006)

[BFGS12] Baldomá, I., Fontich, E., Guardia, M., Seara, T.M.: Exponentially small splitting of separatrices beyond Melnikov analysis: rigorous results. J. Differ. Equ. 253(12), 3304-3439 (2012)

[Cas57] Cassels, J.W.S.: An Introduction to Diophantine Approximation. Cambridge University Press, Cambridge (1957)

[Cha02] Chandre, C.: Renormalization for cubic frequency invariant tori in Hamiltonian systems with two degrees of freedom. Discrete Contin. Dyn. Syst. Ser. B 2(3), 457-465 (2002)

[DG00] Delshams, A., Gutiérrez, P.: Splitting potential and the Poincaré-Melnikov method for whiskered tori in Hamiltonian systems. J. Nonlinear Sci. 10(4), 433-476 (2000)

[DG01] Delshams, A., Gutiérrez, P.: Homoclinic orbits to invariant tori in Hamiltonian systems. In: Jones, C.K.R.T., Khibnik, A.I. (eds.) Multiple-Time-Scale Dynamical Systems (Minneapolis, MN, 1997), Volume 122 of IMA Volumes in Mathematics and its Applications, pp. 1-27. Springer, New York (2001)

[DG03] Delshams, A., Gutiérrez, P.: Exponentially small splitting of separatrices for whiskered tori in Hamiltonian systems. Zap. Nauchn. Sem. S.-Peterburg. Otdel. Mat. Inst. Steklov. (POMI) 300, 87-121 (2003). (J. Math. Sci. (N.Y.) 128(2), 2726-2746 (2005))

[DG04] Delshams, A., Gutiérrez, P.: Exponentially small splitting for whiskered tori in Hamiltonian systems: continuation of transverse homoclinic orbits. Discrete Contin. Dyn. Syst. 11(4), 757 $783(2004)$

[DGG14a] Delshams, A., Gonchenko, M., Gutiérrez, P.: Exponentially small asymptotic estimates for the splitting of separatrices to whiskered tori with quadratic and cubic frequencies. Electron. Res. Announc. Math. Sci. 21, 41-61 (2014)

[DGG14b] Delshams, A., Gonchenko, M., Gutiérrez, P.: Exponentially small lower bounds for the splitting of separatrices to whiskered tori with frequencies of constant type. Int. J. Bifur. Chaos Appl. Sci. Eng. 24(8), 1440011 (2014)

[DGG14c] Delshams, A., Gonchenko, M., Gutiérrez, P.: Continuation of the exponentially small transversality for the splitting of separatrices to a whiskered torus with silver ratio. Regul. Chaotic Dyn. 19(6), 663-680 (2014)

[DGG16] Delshams, A., Gonchenko, M., Gutiérrez, P.: Exponentially small splitting of separatrices and transversality associated to whiskered tori with quadratic frequency ratio. SIAM J. Appl. Dyn. Syst. 15(2), 981-1024 (2016)

[DGJS97] Delshams, A., Gelfreich, V., Jorba, À., Seara, T.M.: Exponentially small splitting of separatrices under fast quasiperiodic forcing. Commun. Math. Phys. 189, 35-71 (1997)

[DGS04] Delshams, A., Gutiérrez, P., Seara, T.M.: Exponentially small splitting for whiskered tori in Hamiltonian systems: flow-box coordinates and upper bounds. Discrete Contin. Dyn. Syst. 11(4), 785-826 (2004)

[DLS06] Delshams, A., de la Llave, R., Seara, T.M.: A geometric mechanism for diffusion in Hamiltonian systems overcoming the large gap problem: heuristics and rigorous verification on a model. Mem. Am. Math. Soc. 179(844) (2006)

[DR98] Delshams, A., Ramírez-Ros, R.: Exponentially small splitting of separatrices for perturbed integrable standard-like maps. J. Nonlinear Sci. 8(3), 317-352 (1998)

[DS92] Delshams, A., Seara, T.M.: An asymptotic expression for the splitting of separatrices of the rapidly forced pendulum. Commun. Math. Phys. 150, 433-463 (1992)

[DS97] Delshams, A., Seara, T.M.: Splitting of separatrices in Hamiltonian systems with one and a half degrees of freedom. Math. Phys. Electron. J. 3, paper 4 (1997)

[Eli94] Eliasson, L.H.: Biasymptotic solutions of perturbed integrable Hamiltonian systems. Bol. Soc. Brasil. Mat. (N.S.) 25(1), 57-76 (1994)

[FSV18a] Fontich, E., Simó, C., Vieiro, A.: On the 'hidden' harmonics associated to best approximants due to quasi-periodicity in splitting phenomena. Regul. Chaotic Dyn. 23(6), 638-653 (2018)

[FSV18b] Fontich, E., Simó, C., Vieiro, A.: Splitting of the separatrices after a Hamiltonian-Hopf bifurcation under periodic forcing. Nonlinearity 32(4), 1440-1493 (2019)

[Ge197] Gelfreich, V.: Melnikov method and exponentially small splitting of separatrices. Physica D 101(3-4), 227-248 (1997)

[GGM99] Gallavotti, G., Gentile, G., Mastropietro, V.: Melnikov approximation dominance. Some examples. Rev. Math. Phys. 11(4), 451-461 (1999)

[GL06] Gidea, M., de la Llave, R.: Topological methods in the instability problem of Hamiltonian systems. Discrete Contin. Dyn. Syst. 14(2), 295-328 (2006) 
[GR03] Gidea, M., Robinson, C.: Topologically crossing heteroclinic connections to invariant tori. J. Differ. Equ. 193(1), 49-74 (2003)

[GS12] Guardia, M., Seara, T.M.: Exponentially and non-exponentially small splitting of separatrices for the pendulum with a fast meromorphic perturbation. Nonlinearity 25(5), 1367-1412 (2012)

[HK00] Hardcastle, D.M., Khanin, K.: On almost everywhere strong convergence of multi-dimensional continued fraction algorithms. Ergodic Theory Dyn. Syst. 20(6), 1711-1733 (2000)

[HMS88] Holmes, P., Marsden, J.E., Scheurle, J.: Exponentially small splittings of separatrices with applications to KAM theory and degenerate bifurcations. In: Hamiltonian Dynamical Systems (Boulder, CO, 1987), Volume 81 of Contemporary Mathematics, pp. 213-244. American Mathematical Society, Providence, RI (1988)

[Koc99] Koch, H.: A renormalization group for Hamiltonians, with applications to KAM theory. Ergodic Theory Dyn. Syst. 19(2), 475-521 (1999)

[Lan02] Lang, S.: Algebra Volume 211 of Graduate Texts in Mathematics, 3rd edn. Springer, New York (2002)

[Laz03] Lazutkin, V.F.: Splitting of separatrices for the Chirikov standard map. Zap. Nauchn. Sem. S.Peterburg. Otdel. Mat. Inst. Steklov. (POMI) 300, 25-55 (2003). (J. Math. Sci. (N.Y.) 128(2), 2687-2705 (2005). The original Russian preprint appeared in 1984)

[LMS03] Lochak, P., Marco, J.-P., Sauzin, D.: On the splitting of invariant manifolds in multidimensional near-integrable Hamiltonian systems. Mem. Am. Math. Soc. 163(775) (2003)

[Loc90] Lochak, P.: Effective speed of Arnold's diffusion and small denominators. Phys. Lett. A 143(12), 39-42 (1990)

[Loc92] Lochak, P.: Canonical perturbation theory via simultaneous approximation. Russ. Math. Surv. 47(6), 57-133 (1992). (Uspekhi Mat. Nauk 47(6), 59-140 (1992))

[Lop02] Lopes Dias, J.: Renormalization of flows on the multidimensional torus close to a KT frequency vector. Nonlinearity 15(3), 647-664 (2002)

[Me163] Melnikov, V.K.: On the stability of the center for time periodic perturbations. Trans. Moscow Math. Soc. 12, 1-57 (1963). (Trudy Moskov. Mat. Obšč. 12, 3-52 (1963))

[Nei84] Neishtadt, A.I.: The separation of motions in systems with rapidly rotating phase. J. Appl. Math. Mech. 48(2), 133-139 (1984) (Prikl. Mat. Mekh. 48(2), 197-204 (1984))

[Nek77] Nekhoroshev, N.N.: An exponential estimate of the time of stability of nearly-integrable Hamiltonian systems. Russ. Math. Surv. 32(6), 1-65 (1977) (Uspekhi Mat. Nauk 32(6), 5-66 (1977))

[Nie00] Niederman, L.: Dynamics around simple resonant tori in nearly integrable Hamiltonian systems. J. Differ. Equ. 161(1), 1-41 (2000)

[Poi90] Poincaré, H.: Sur le problème des trois corps et les équations de la dynamique. Acta Math. 13, $1-270(1890)$

[PT00] Pronin, A., Treschev, D.V.: Continuous averaging in multi-frequency slow-fast systems. Regul. Chaotic Dyn. 5(2), 157-170 (2000)

[RW00] Rudnev, M., Wiggins, S.: On a homoclinic splitting problem. Regul. Chaotic Dyn. 5(2), 227-242 (2000)

[Sau01] Sauzin, D.: A new method for measuring the splitting of invariant manifolds. Ann. Sci. École Norm. Sup. (4) 34(2), 159-221 (2001)

[Sch80] Schmidt, W.M.: Diophantine Approximation, Volume 785 of Lecture Notes in Mathematics. Springer, Berlin (1980)

[Sch89] Scheurle, J.: Chaos in a rapidly forced pendulum equation. In: Dynamics and Control of Multibody Systems (Brunswick, ME, 1988), Volume 97 of Contemporary Mathematics, pp. 411-419. American Mathematical Society, Providence, RI (1989)

[Sim94] Simó, C.: Averaging under fast quasiperiodic forcing. In: Seimenis, J. (ed.) Hamiltonian Mechanics: Integrability and Chaotic Behavior (Toruń, 1993), Volume 331 of NATO ASI Series B: Physics, pp. 13-34. Plenum, New York (1994)

[ST87] Stewart, I., Tall, D.: Algebraic Number Theory, 2nd edn. Chapman \& Hall, London (1987)

[Tre94] Treschev, D.V.: Hyperbolic tori and asymptotic surfaces in Hamiltonian systems. Russ. J. Math. Phys. 2(1), 93-110 (1994)

[Tre97] Treschev, D.V.: Splitting of separatrices for a pendulum with rapidly oscillating suspension point. Russ. J. Math. Phys. 5(1), 63-98 (1997)

[Wei03] Weisstein, E.W.: CRC Concise Encyclopedia of Mathematics, 2nd edn. CRC Press, Boca Raton, FL (2003) 